Supporting Information

\title{
Copper-catalyzed Asymmetric 1,3-Dipolar Cycloaddition of Iminoesters to Unsaturated Sultones
}

\author{
Shohei Furuya, Kazuya Kanemoto,* and Shin-ichi Fukuzawa* \\ Department of Applied Chemistry, Institute of Science and Engineering, Chuo University 1-13-27 \\ Kasuga, Bunkyo-ku, Tokyo 112-8551, Japan \\ E-mail: k-kanemoto.18n@g.chuo-u.ac.jp. orgsynth@kc.chuo-u.ac.jp.
}

\section{Contents}

${ }^{1} \mathrm{H}$ and ${ }^{13} \mathrm{C}$ NMR Spectra of Compounds $\quad$ S2

HPLC Spectra of Compounds $\quad$ S26

X-Ray Crystal Structure Analysis $\quad$ S50

References for Supporting Information $\quad$ S54 


\section{${ }^{1} \mathrm{H}$ and ${ }^{13} \mathrm{C}$ NMR Spectra of Compounds}

${ }^{1} \mathrm{H}$ NMR $(400 \mathrm{MHz})$ and ${ }^{13} \mathrm{C}$ NMR $(101 \mathrm{MHz})$ spectra of $\mathbf{4 a}\left(\mathrm{CD}_{3} \mathrm{CN}\right)$

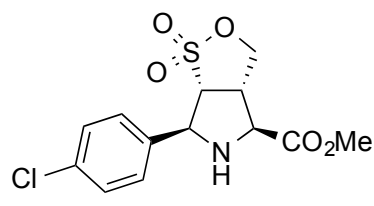

4a
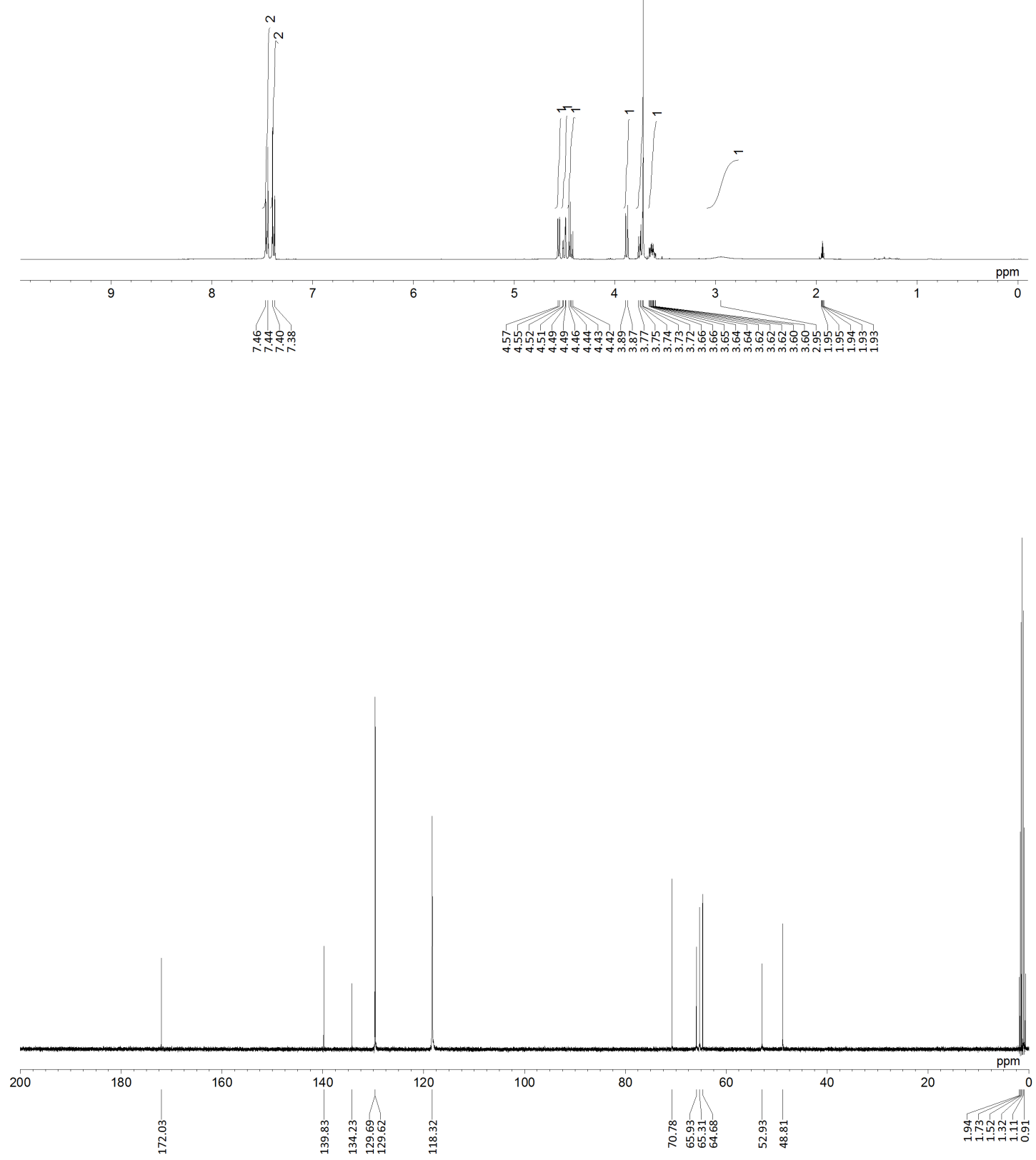
${ }^{1} \mathrm{H}$ NMR $(400 \mathrm{MHz})$ and ${ }^{13} \mathrm{C}$ NMR $(101 \mathrm{MHz})$ spectra of $\mathbf{4 b}\left(\mathrm{CD}_{3} \mathrm{CN}\right)$

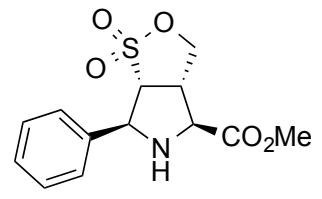

4b
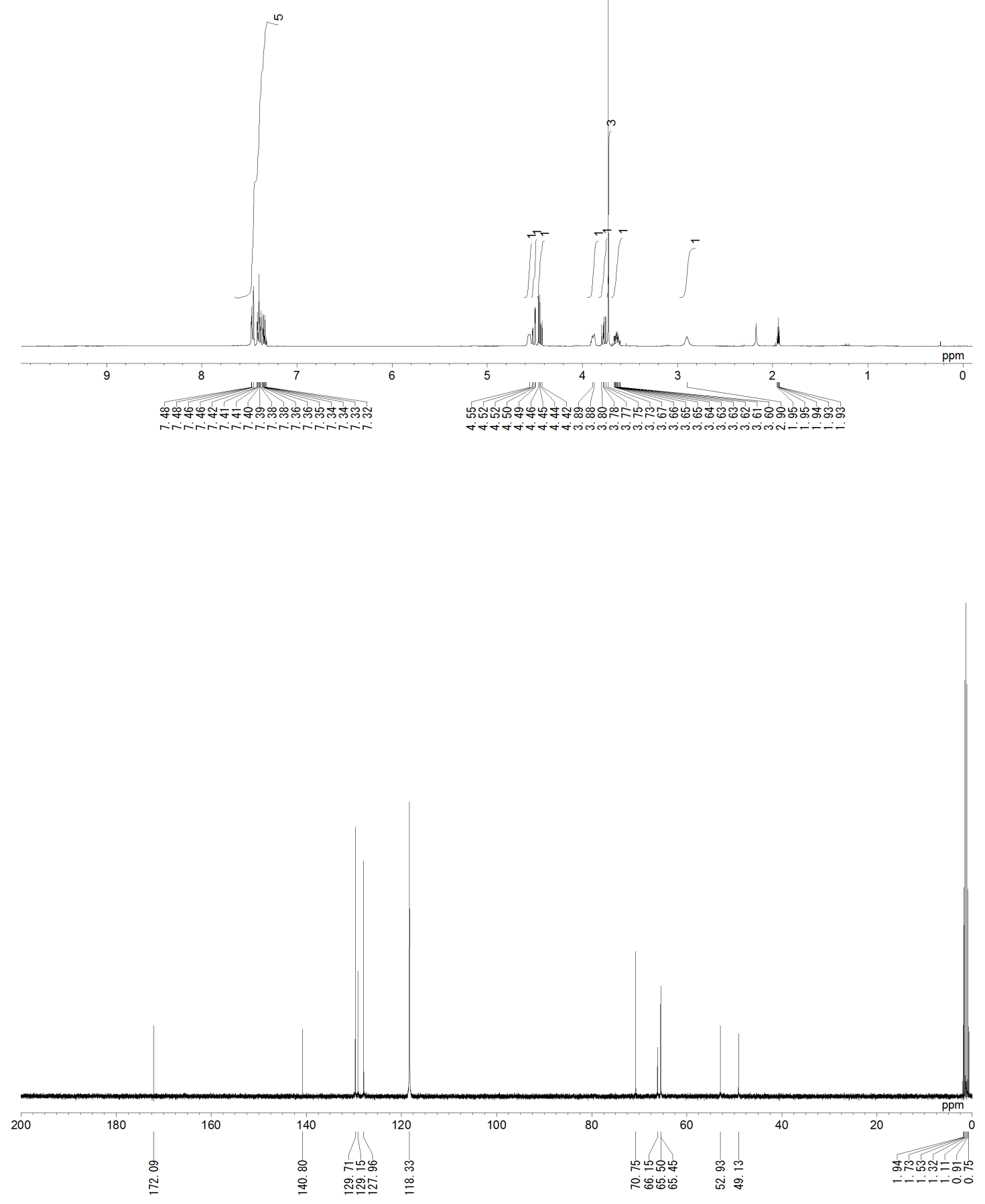
${ }^{1} \mathrm{H}$ NMR $(400 \mathrm{MHz})$ and ${ }^{13} \mathrm{C}$ NMR $(101 \mathrm{MHz})$ spectra of $\mathbf{4 c}\left(\mathrm{CDCl}_{3}\right)$
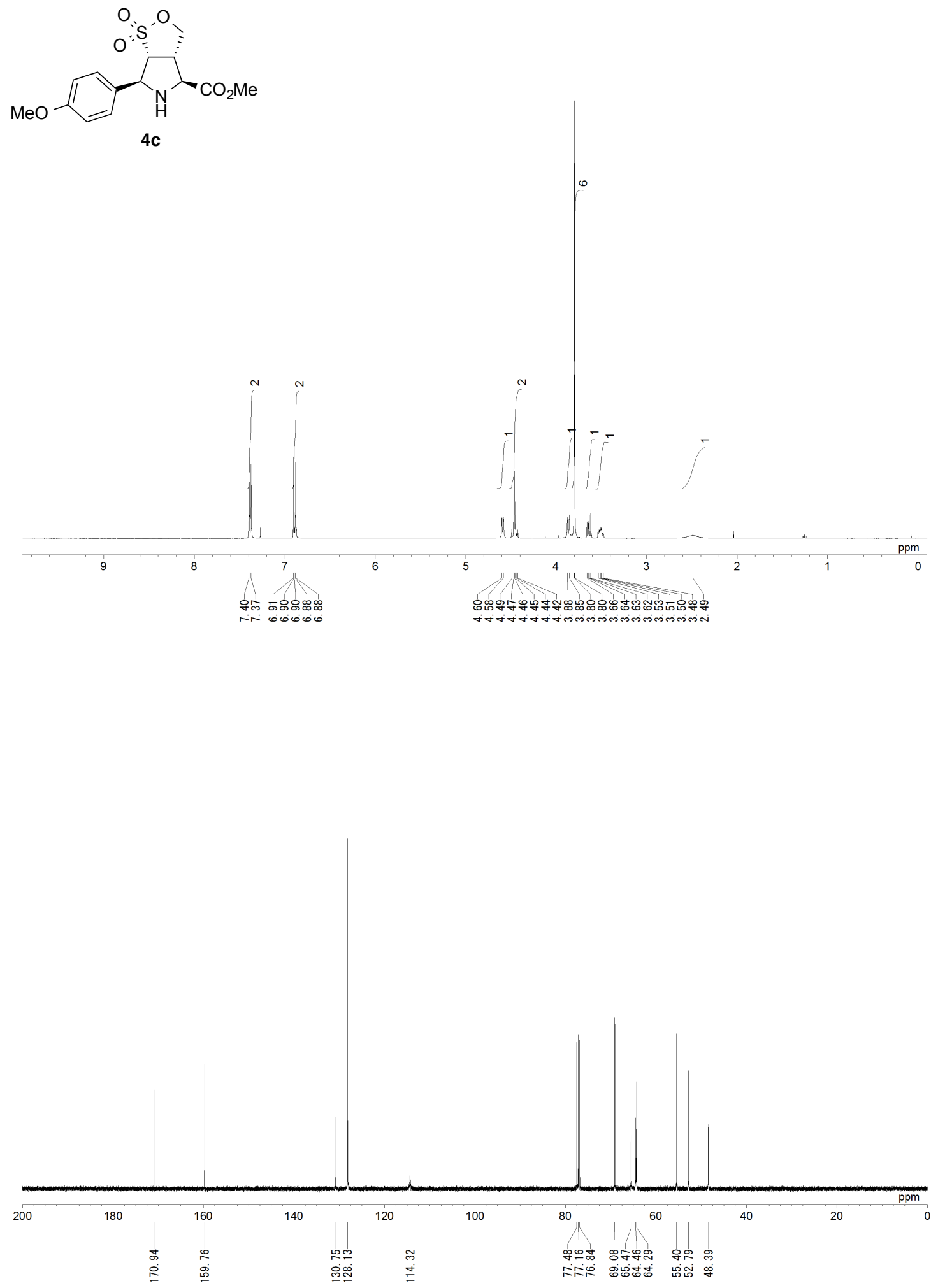
${ }^{1} \mathrm{H}$ NMR $(400 \mathrm{MHz})$ and ${ }^{13} \mathrm{C}$ NMR $(101 \mathrm{MHz})$ spectra of $\mathbf{4 d}\left(\mathrm{CDCl}_{3}\right)$

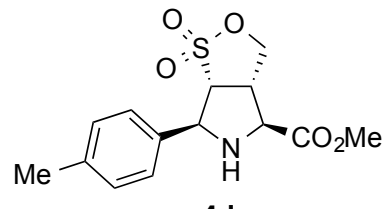

4d
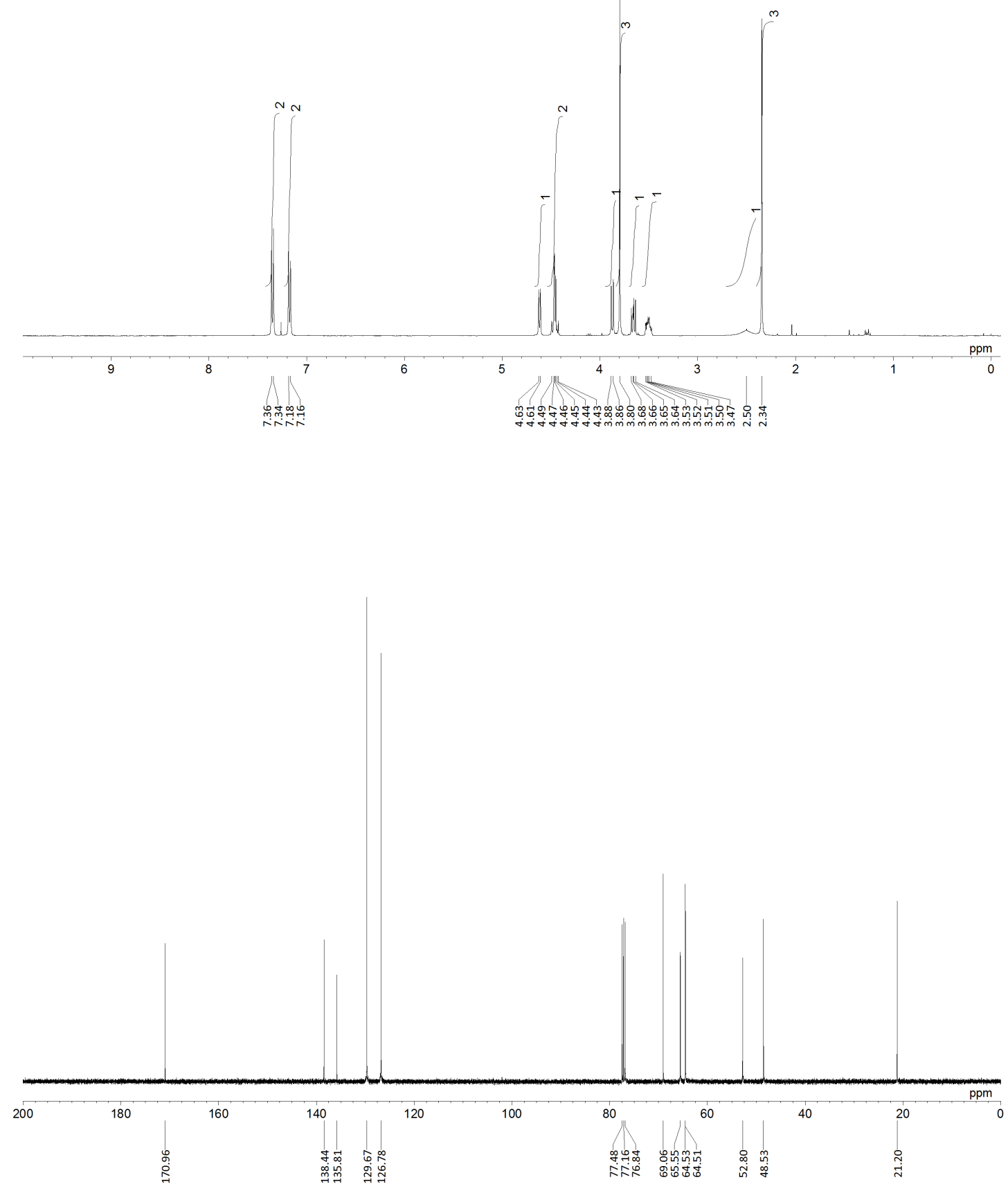
${ }^{1} \mathrm{H}$ NMR $(400 \mathrm{MHz})$ and ${ }^{13} \mathrm{C}$ NMR $(101 \mathrm{MHz})$ spectra of $\mathbf{4 e}\left(\mathrm{CD}_{3} \mathrm{CN}\right)$
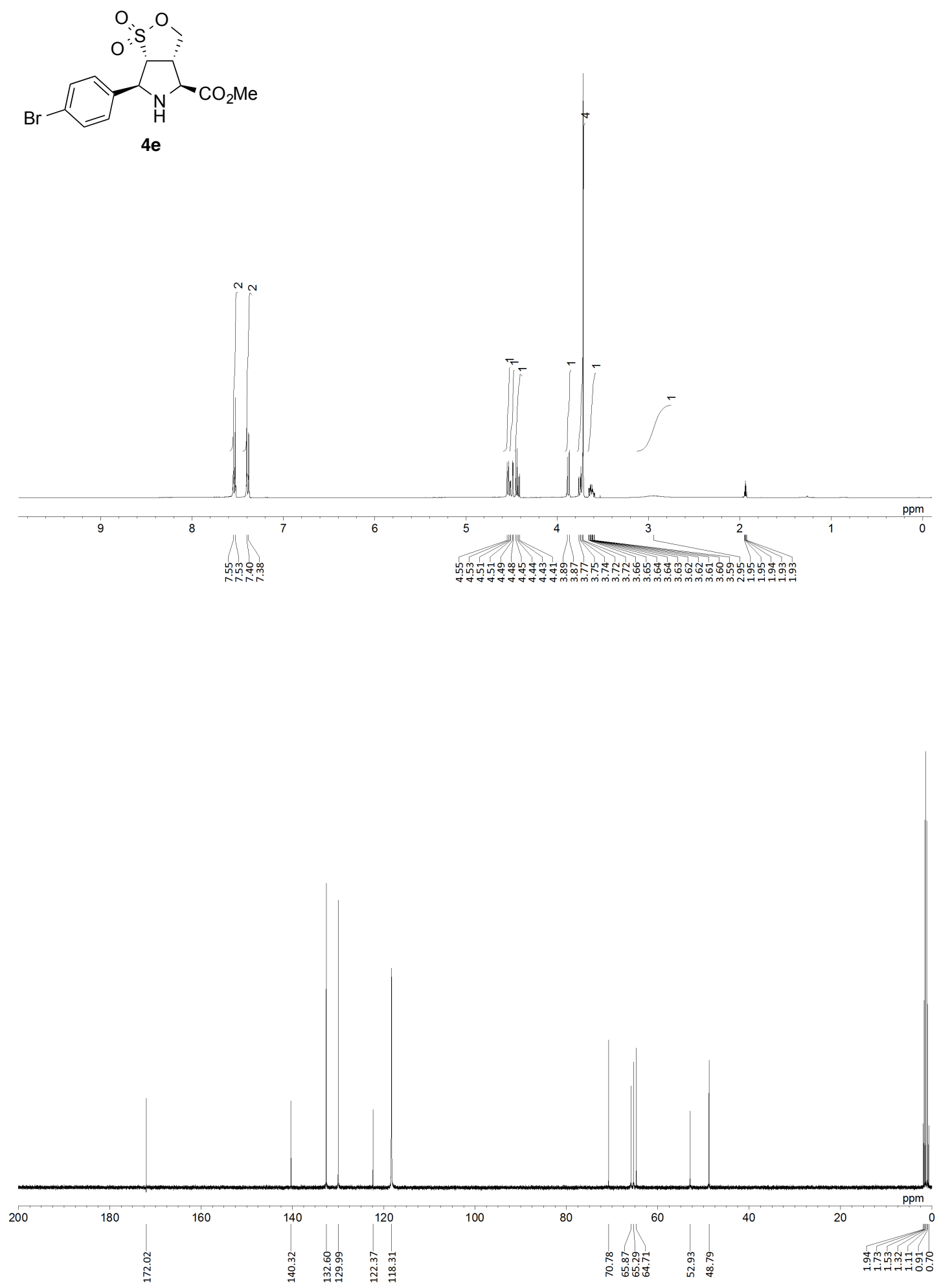
${ }^{1} \mathrm{H}$ NMR $(400 \mathrm{MHz})$ and ${ }^{13} \mathrm{C}$ NMR $(101 \mathrm{MHz})$ spectra of $\mathbf{4} \mathbf{f}\left(\mathrm{CD}_{3} \mathrm{CN}\right)$
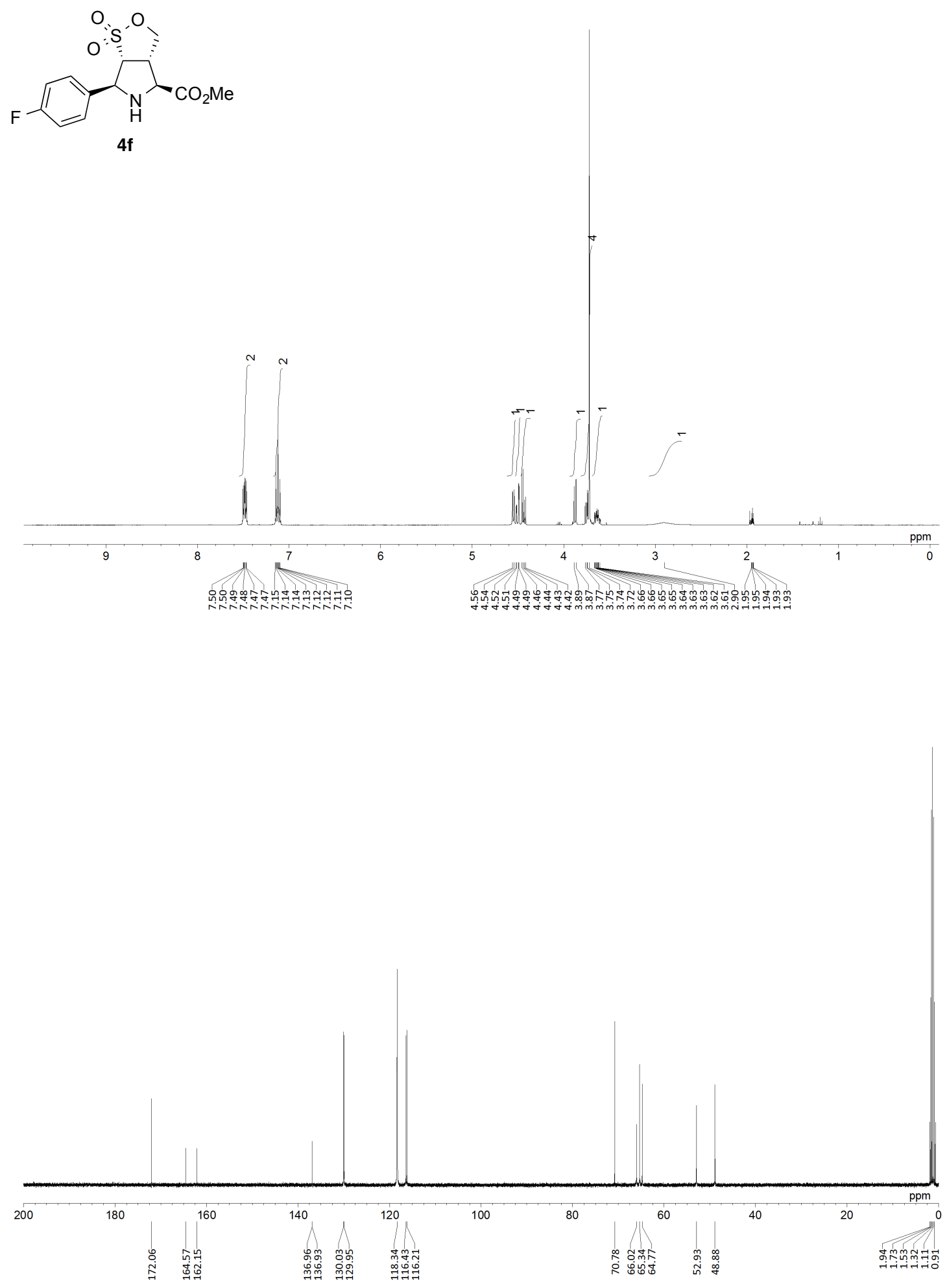
${ }^{1} \mathrm{H}$ NMR (400 MHz) and ${ }^{13} \mathrm{C}$ NMR $(101 \mathrm{MHz})$ spectra of $\mathbf{4 g}\left(\mathrm{CD}_{3} \mathrm{CN}\right)$<smiles>CC(=O)C1NC(c2ccc(C(F)(F)F)cc2)C2C(=O)OC[C@@H]12</smiles>
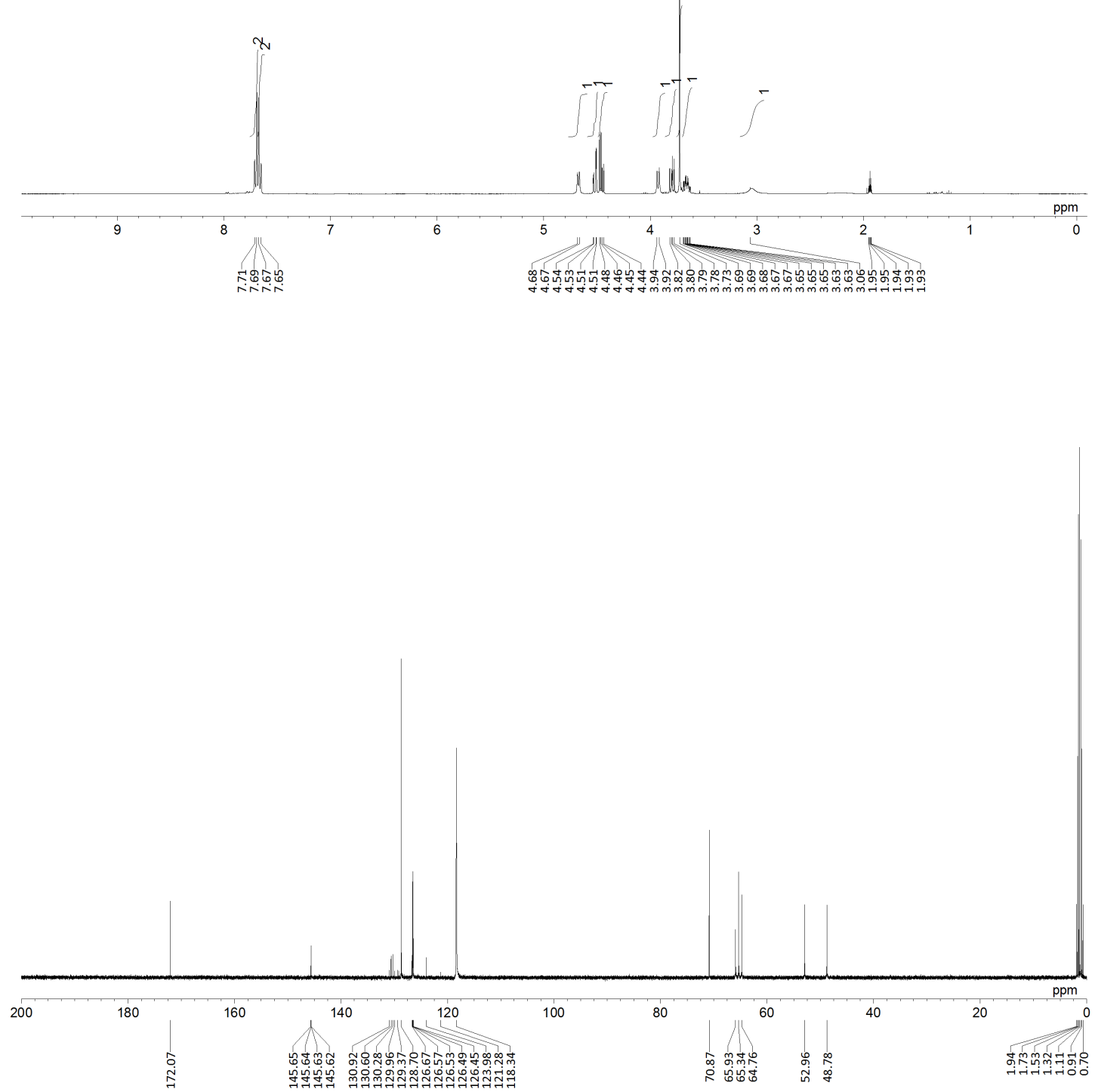
${ }^{1} \mathrm{H}$ NMR $(400 \mathrm{MHz})$ and ${ }^{13} \mathrm{C}$ NMR $(101 \mathrm{MHz})$ spectra of $\mathbf{4 h}\left(\mathrm{CDCl}_{3}\right)$
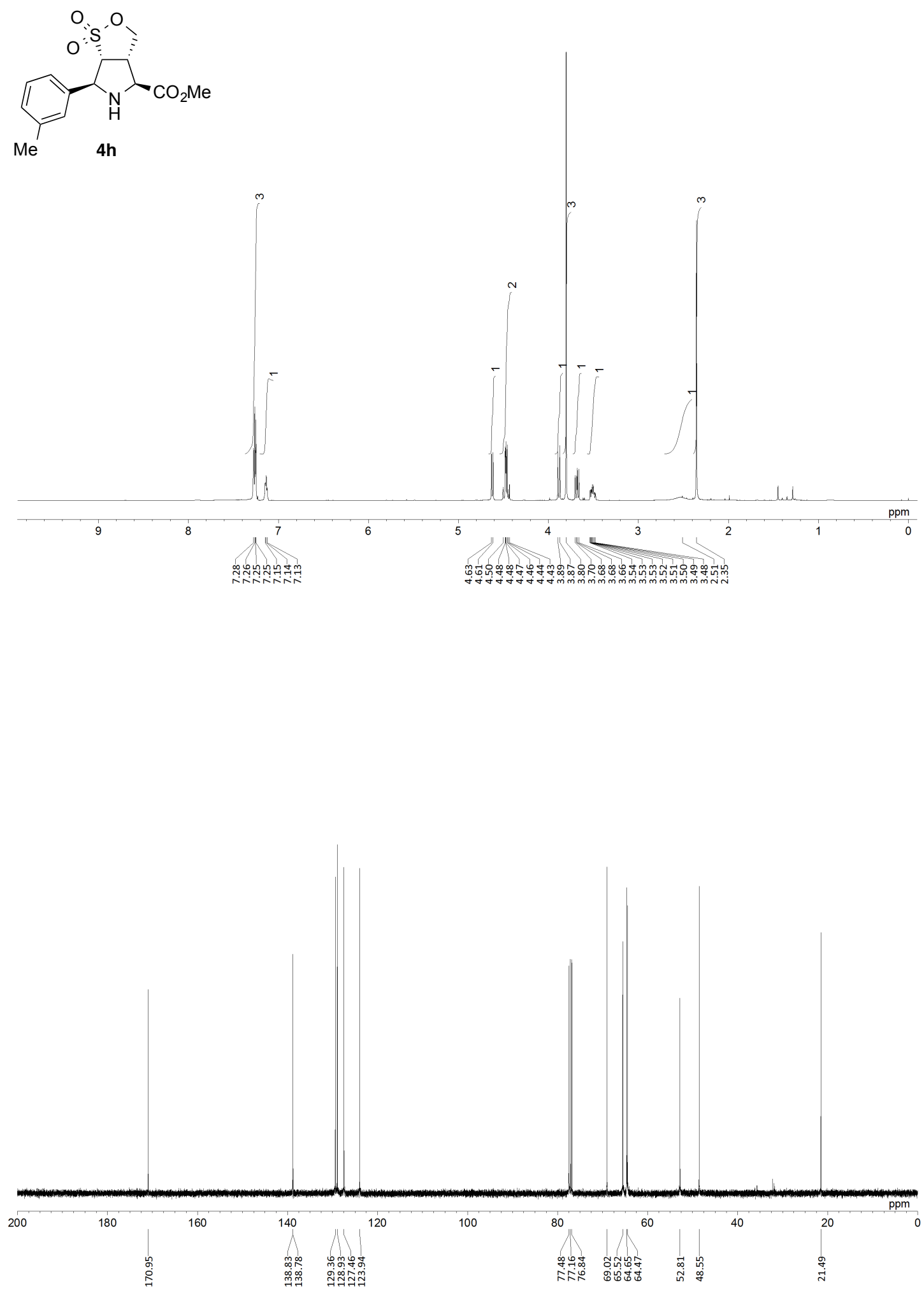
${ }^{1} \mathrm{H}$ NMR $(400 \mathrm{MHz})$ and ${ }^{13} \mathrm{C}$ NMR $(101 \mathrm{MHz})$ spectra of $\mathbf{4 i}\left(\mathrm{CD}_{3} \mathrm{CN}\right)$
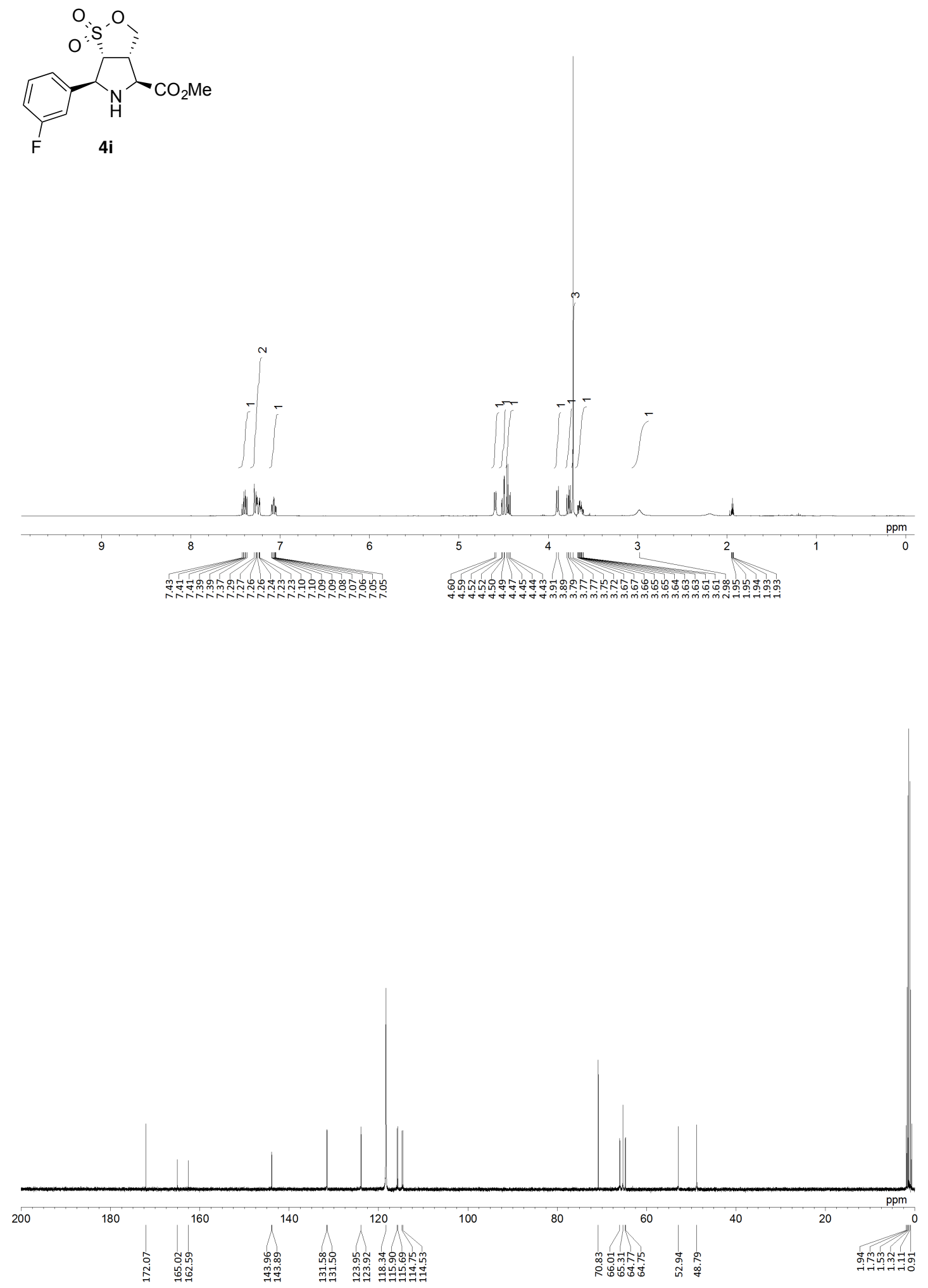
${ }^{1} \mathrm{H}$ NMR $(400 \mathrm{MHz})$ and ${ }^{13} \mathrm{C}$ NMR $(101 \mathrm{MHz})$ spectra of $\mathbf{4 j}\left(\mathrm{CD}_{3} \mathrm{CN}\right)$

${ }^{1} \mathrm{H}$ NMR $(400 \mathrm{MHz})$ and ${ }^{13} \mathrm{C}$ NMR $(101 \mathrm{MHz})$ spectra of $\mathbf{4 k}\left(\mathrm{CDCl}_{3}\right)$
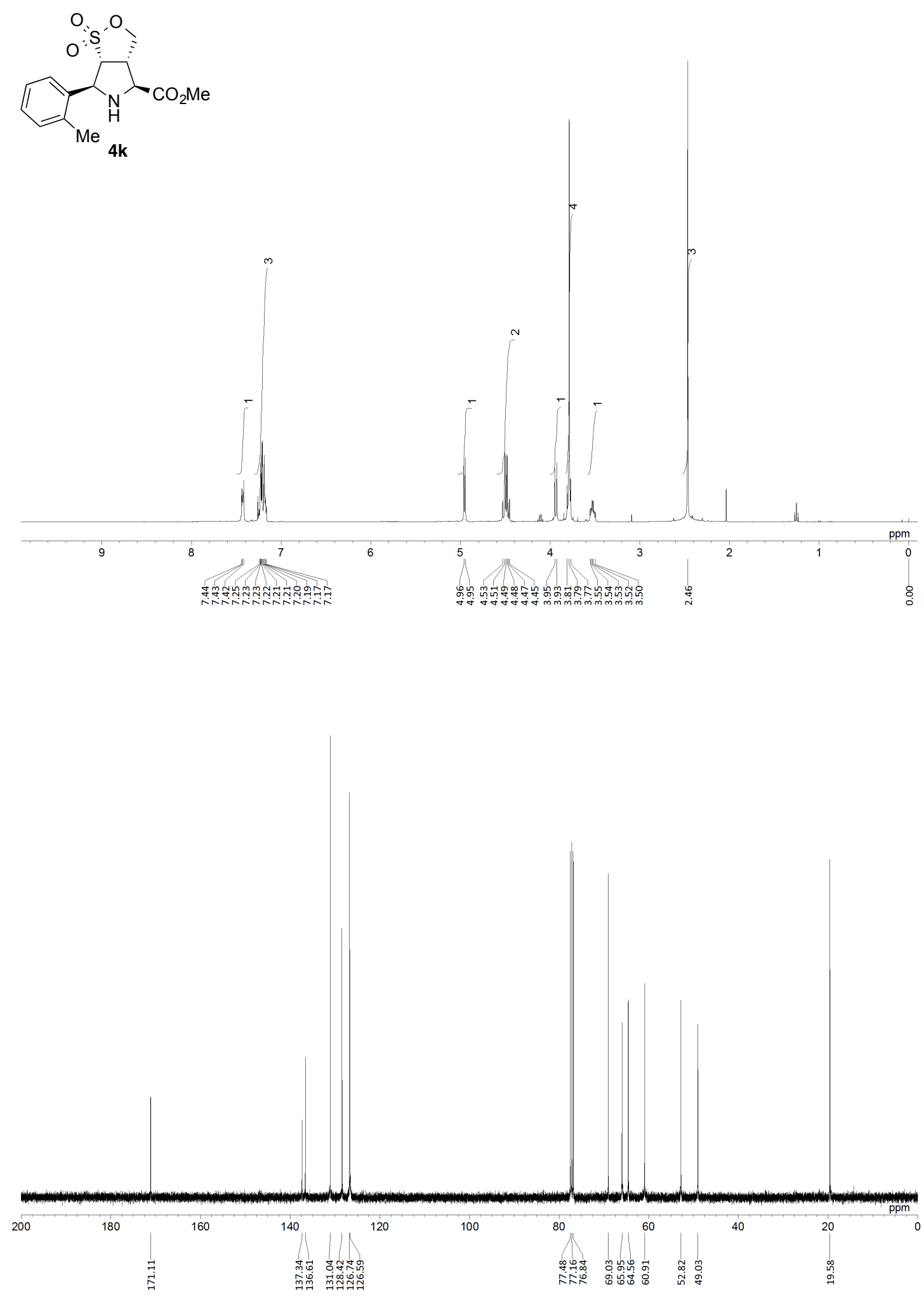
${ }^{1} \mathrm{H}$ NMR $(400 \mathrm{MHz})$ and ${ }^{13} \mathrm{C}$ NMR $(101 \mathrm{MHz})$ spectra of $4 \mathbf{l}\left(\mathrm{CD}_{3} \mathrm{CN}\right)$<smiles>CC(=O)C1NC(c2cccs2)C2COC(=O)[C@@H]12</smiles>

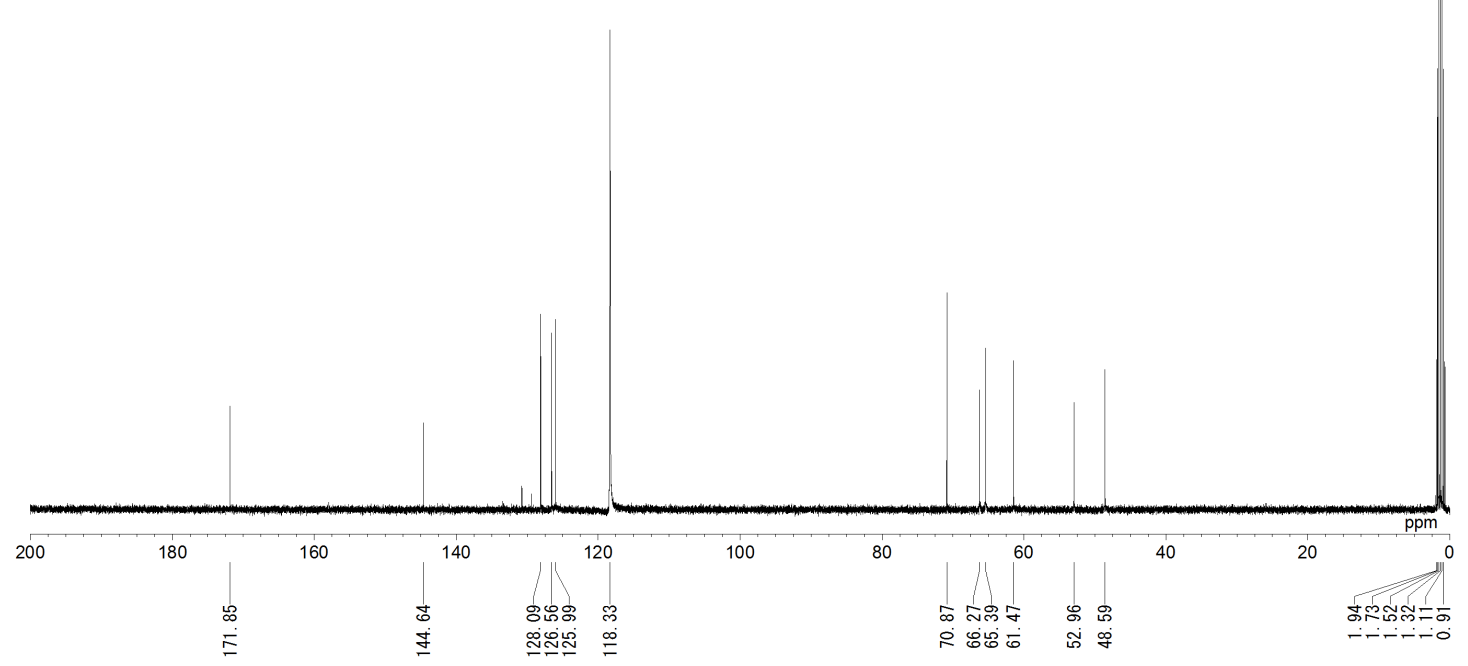


${ }^{1} \mathrm{H}$ NMR $(400 \mathrm{MHz})$ and ${ }^{13} \mathrm{C}$ NMR $(101 \mathrm{MHz})$ spectra of $\mathbf{5 a}\left(\mathrm{CD}_{3} \mathrm{CN}\right)$
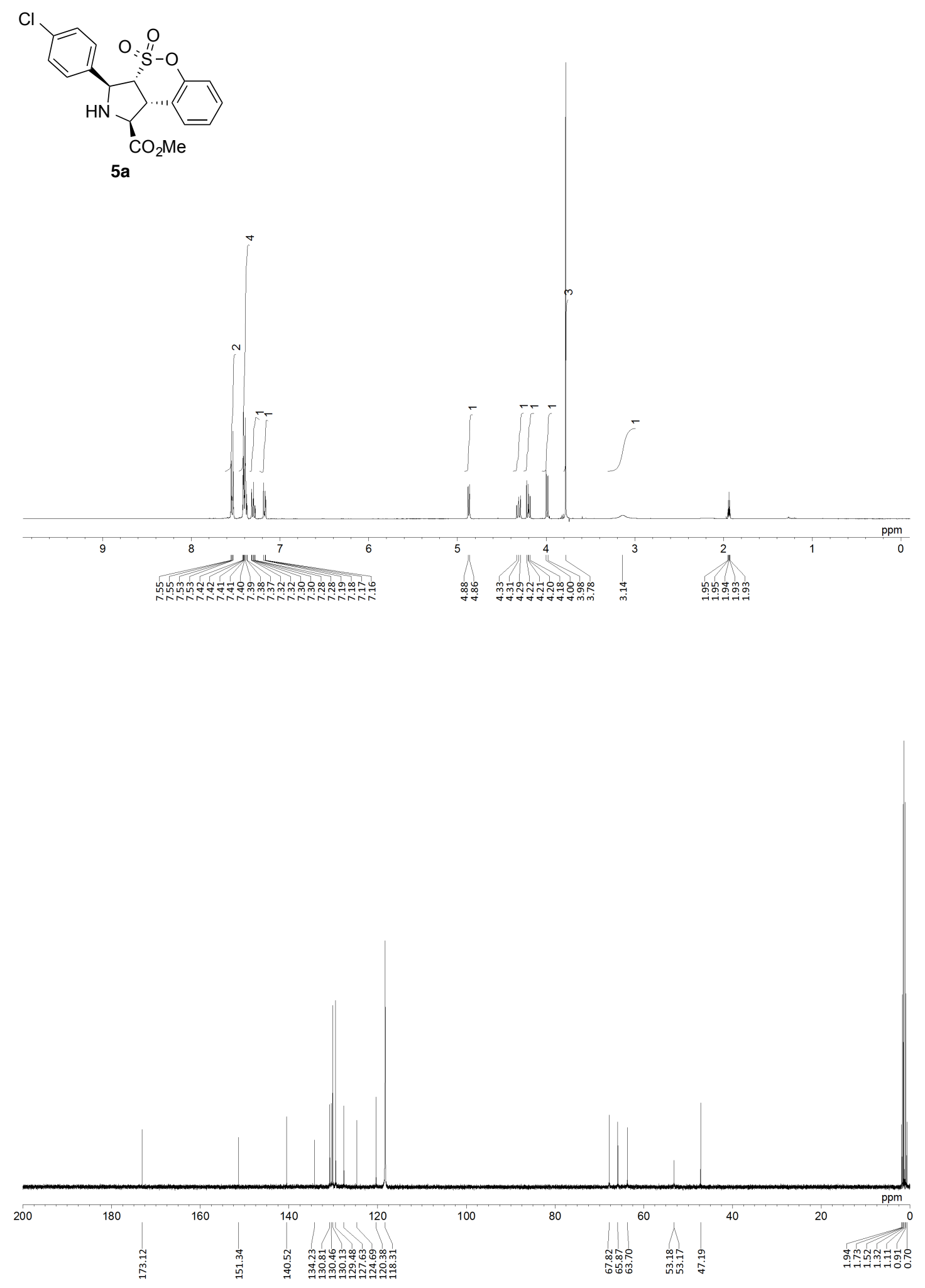
${ }^{1} \mathrm{H}$ NMR $(400 \mathrm{MHz})$ and ${ }^{13} \mathrm{C}$ NMR $(101 \mathrm{MHz})$ spectra of $\mathbf{5 b}\left(\mathrm{CD}_{3} \mathrm{CN}\right)$
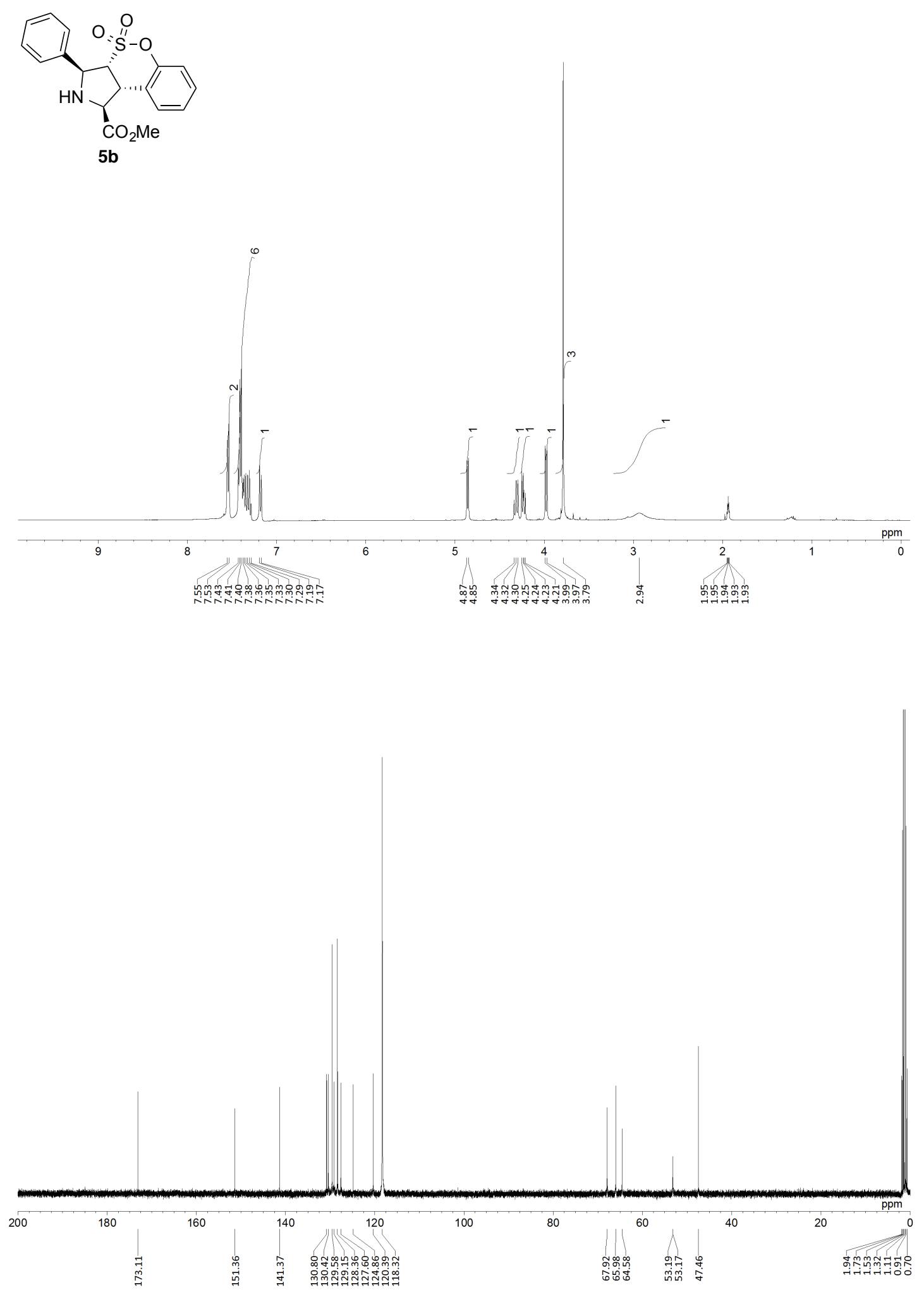
${ }^{1} \mathrm{H}$ NMR $(400 \mathrm{MHz})$ and ${ }^{13} \mathrm{C}$ NMR $(101 \mathrm{MHz})$ spectra of $\mathbf{5 c}\left(\mathrm{CD}_{3} \mathrm{CN}\right)$
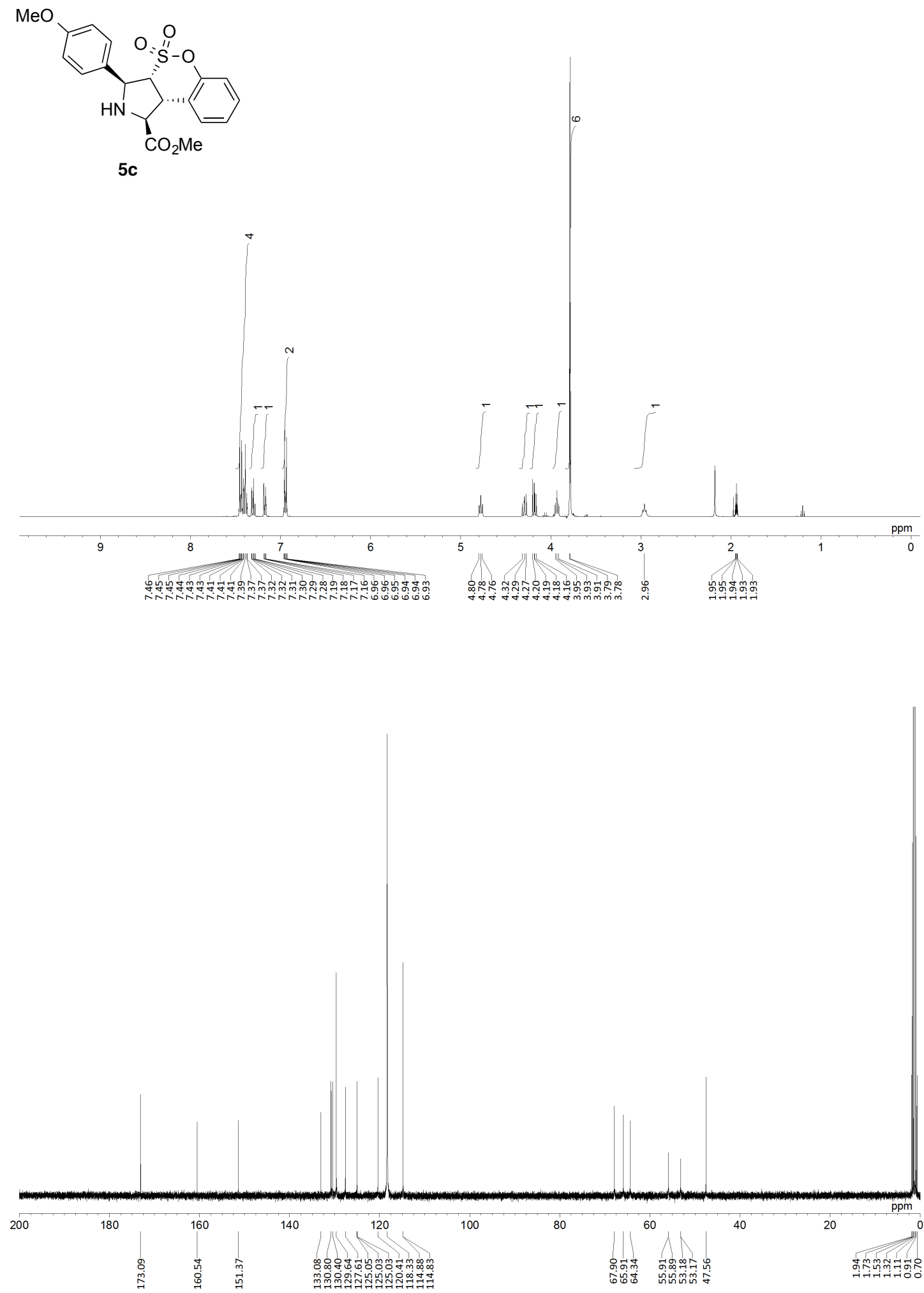
${ }^{1} \mathrm{H}$ NMR $(400 \mathrm{MHz})$ and ${ }^{13} \mathrm{C}$ NMR $(101 \mathrm{MHz})$ spectra of $\mathbf{5 d}\left(\mathrm{CD}_{3} \mathrm{CN}\right)$
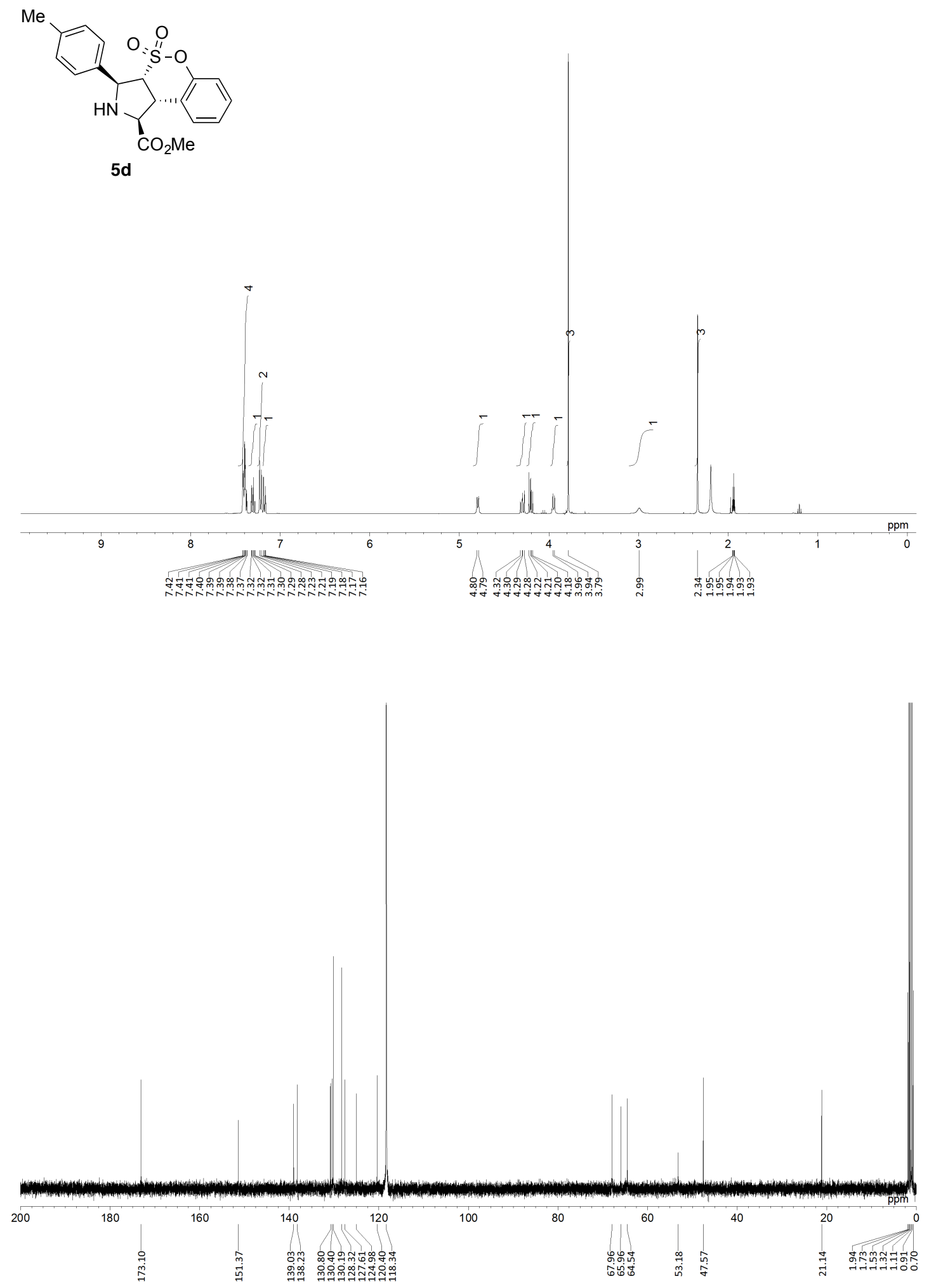
${ }^{1} \mathrm{H}$ NMR $(400 \mathrm{MHz})$ and ${ }^{13} \mathrm{C}$ NMR $(101 \mathrm{MHz})$ spectra of $\mathbf{5 e}\left(\mathrm{CD}_{3} \mathrm{CN}\right)$

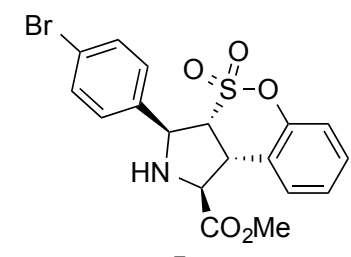

$5 e$
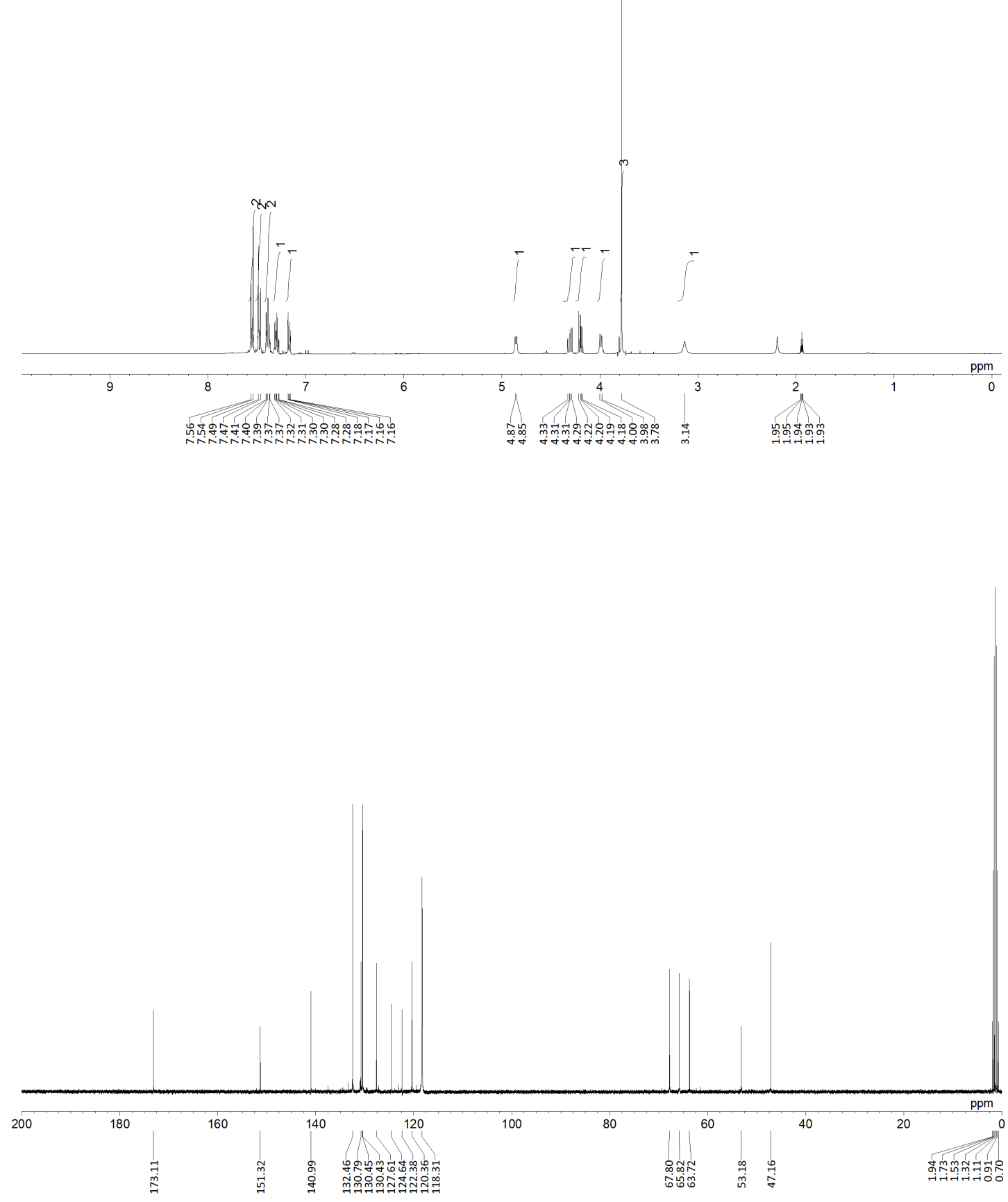
${ }^{1} \mathrm{H}$ NMR $(400 \mathrm{MHz})$ and ${ }^{13} \mathrm{C}$ NMR $(101 \mathrm{MHz})$ spectra of $\mathbf{5 f}\left(\mathrm{CD}_{3} \mathrm{CN}\right)$
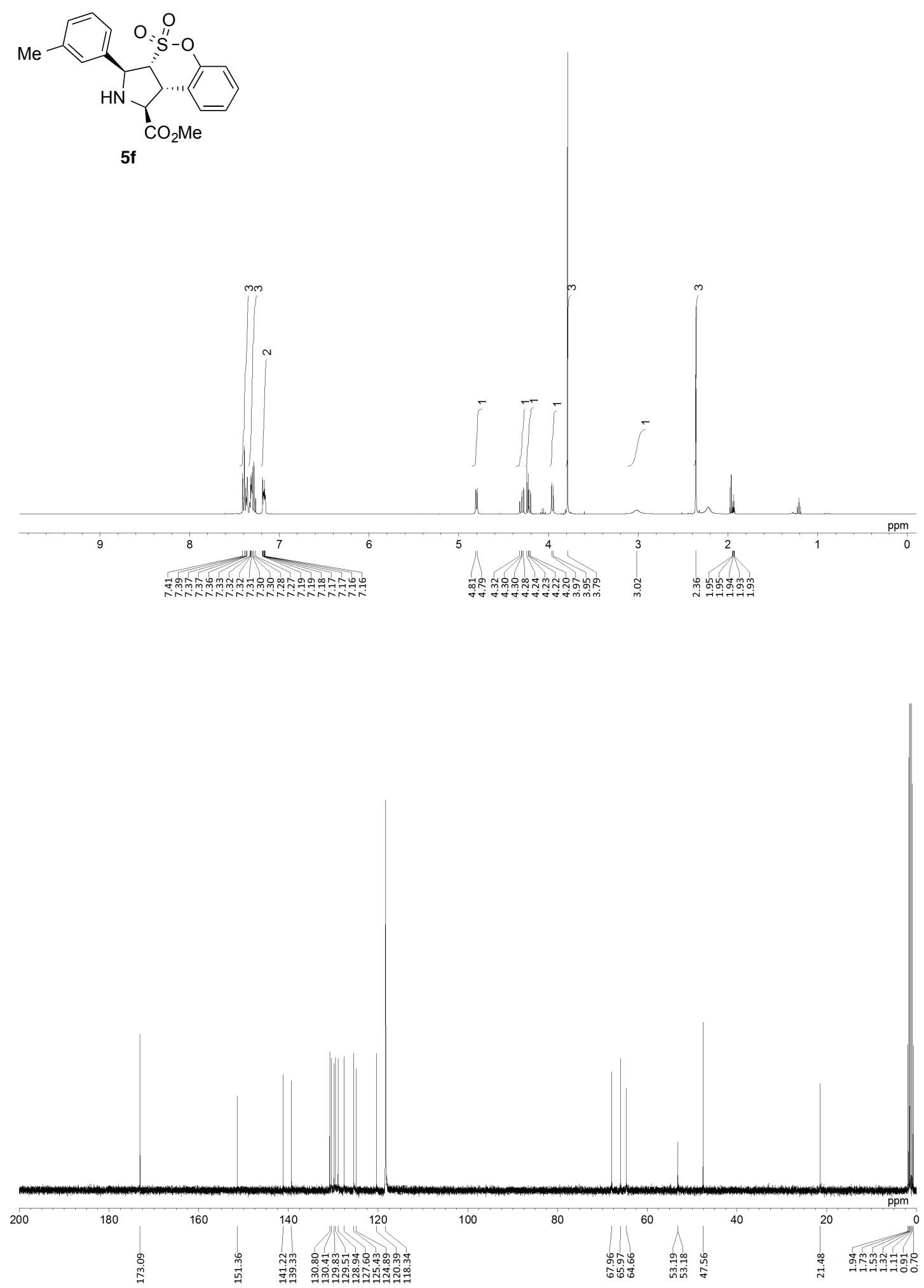
${ }^{1} \mathrm{H}$ NMR $(400 \mathrm{MHz})$ and ${ }^{13} \mathrm{C}$ NMR $(101 \mathrm{MHz})$ spectra of $\mathbf{5 g}\left(\mathrm{CD}_{3} \mathrm{CN}\right)$<smiles>COC(=O)C1NC(c2ccccc2C(C)(C)C)C(S(=O)(=O)Oc2ccccc2)C1c1ccccc1</smiles>

$5 \mathrm{~g}$
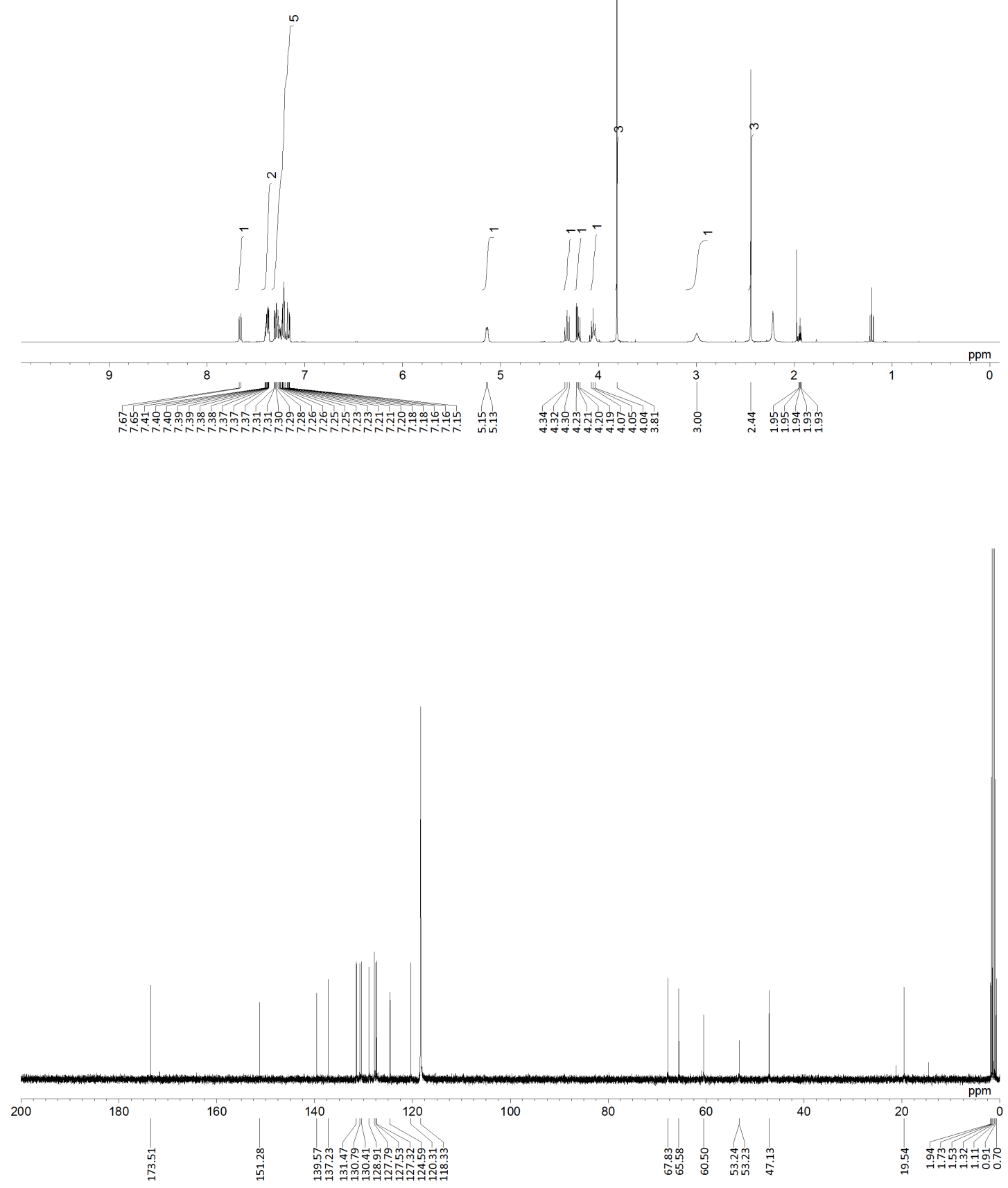
${ }^{1} \mathrm{H}$ NMR $(400 \mathrm{MHz})$ and ${ }^{13} \mathrm{C}$ NMR $(101 \mathrm{MHz})$ spectra of $\mathbf{5} \mathbf{h}\left(\mathrm{CD}_{3} \mathrm{CN}\right)$

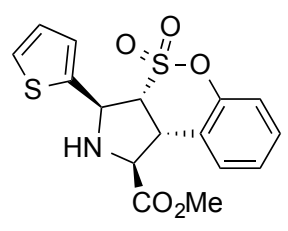

$5 \mathrm{~h}$

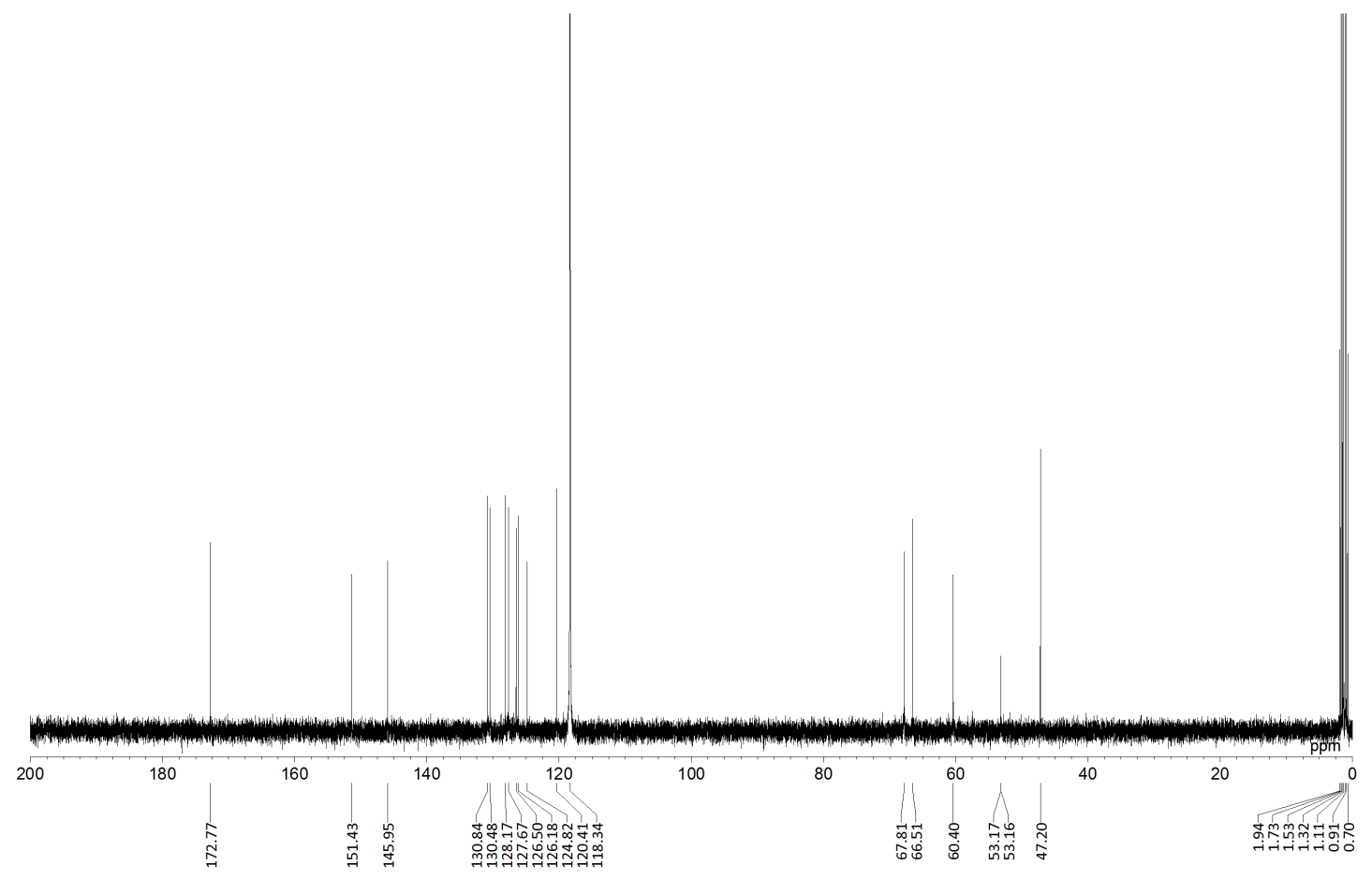


${ }^{1} \mathrm{H}$ NMR (400 MHz) and ${ }^{13} \mathrm{C}$ NMR (101 MHz) spectra of $\mathbf{5 i}$ (DMSO- $d_{6}$ )
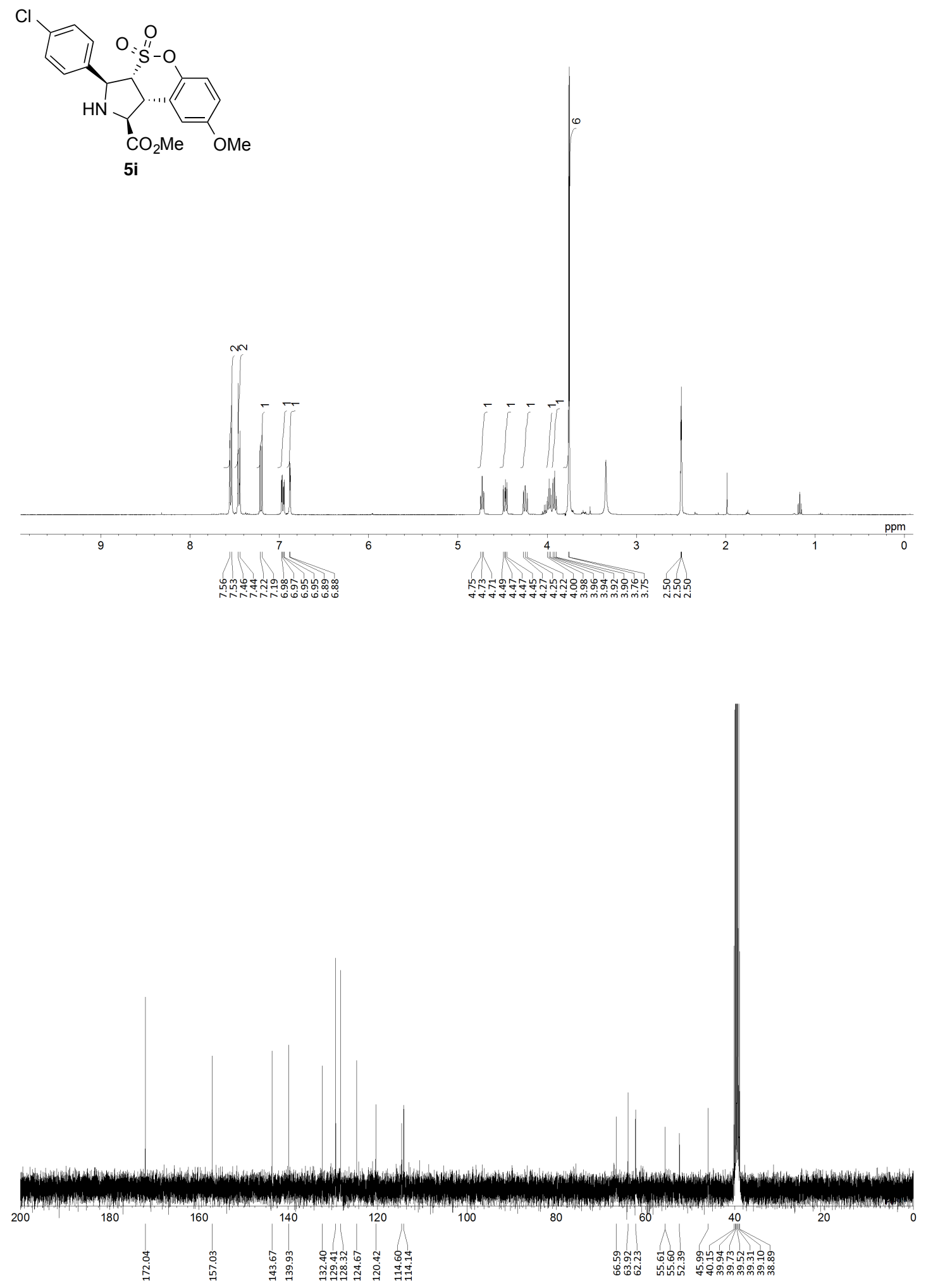
${ }^{1} \mathrm{H}$ NMR (400 MHz) and ${ }^{13} \mathrm{C}$ NMR (101 MHz) spectra of $\mathbf{5 j}$ (DMSO- $d_{6}$ )
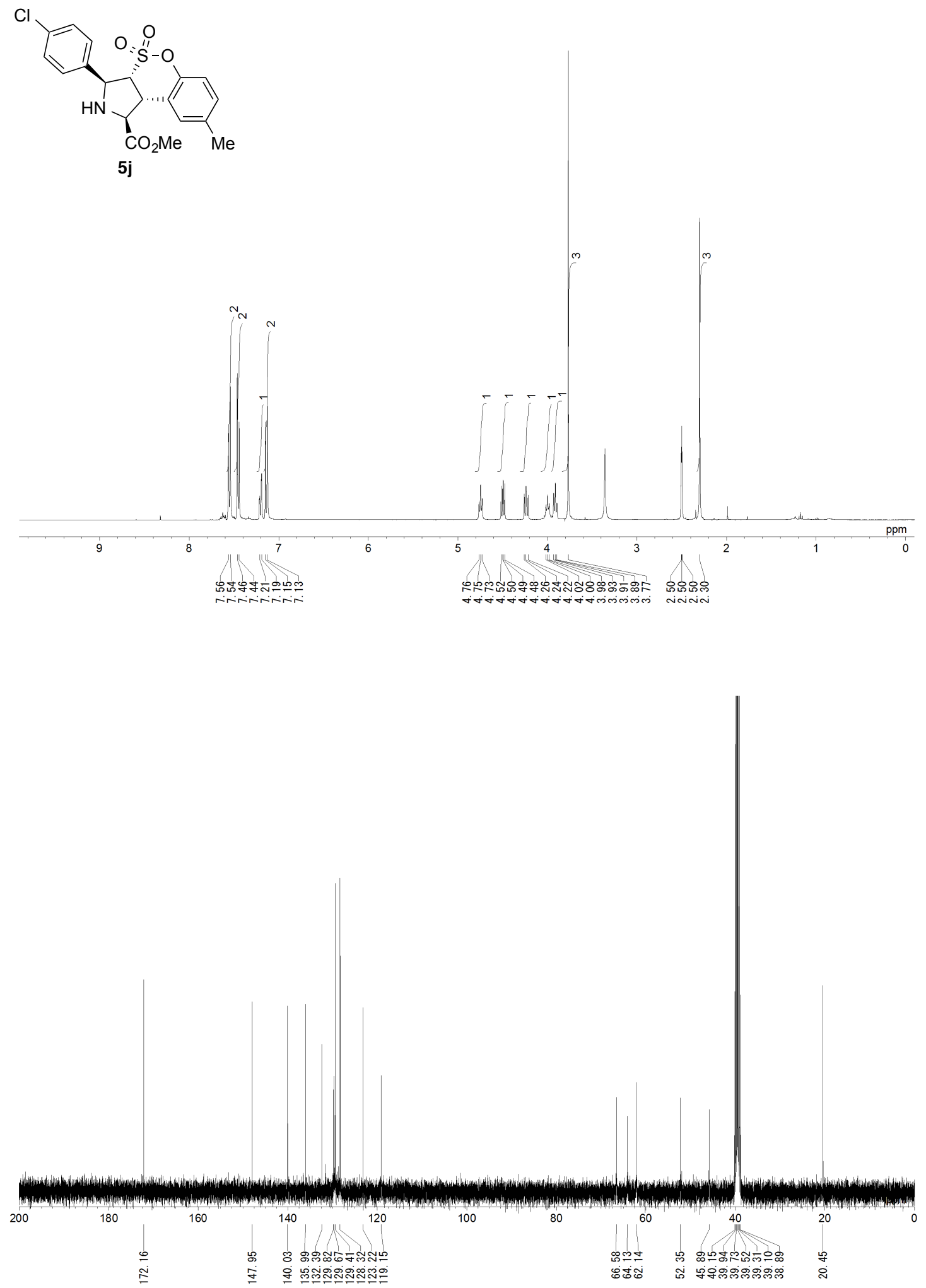
${ }^{1} \mathrm{H}$ NMR $(400 \mathrm{MHz})$ and ${ }^{13} \mathrm{C}$ NMR $(101 \mathrm{MHz})$ spectra of $\mathbf{5 k}$ (DMSO- $\left.d_{6}\right)$
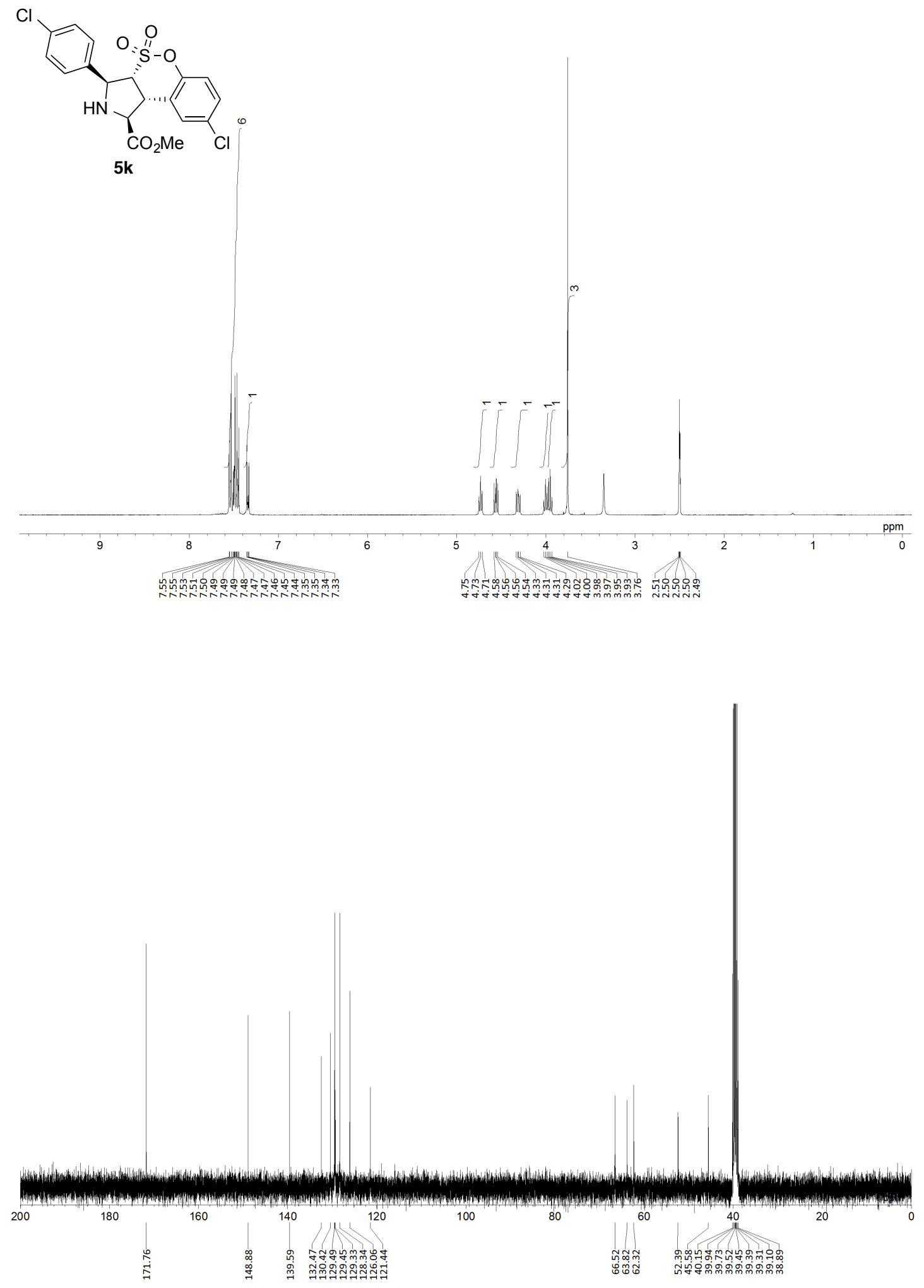
${ }^{1} \mathrm{H}$ NMR $(400 \mathrm{MHz})$ and ${ }^{13} \mathrm{C}$ NMR $(101 \mathrm{MHz})$ spectra of $\mathbf{5 l}\left(\mathrm{CD}_{3} \mathrm{CN}\right)$
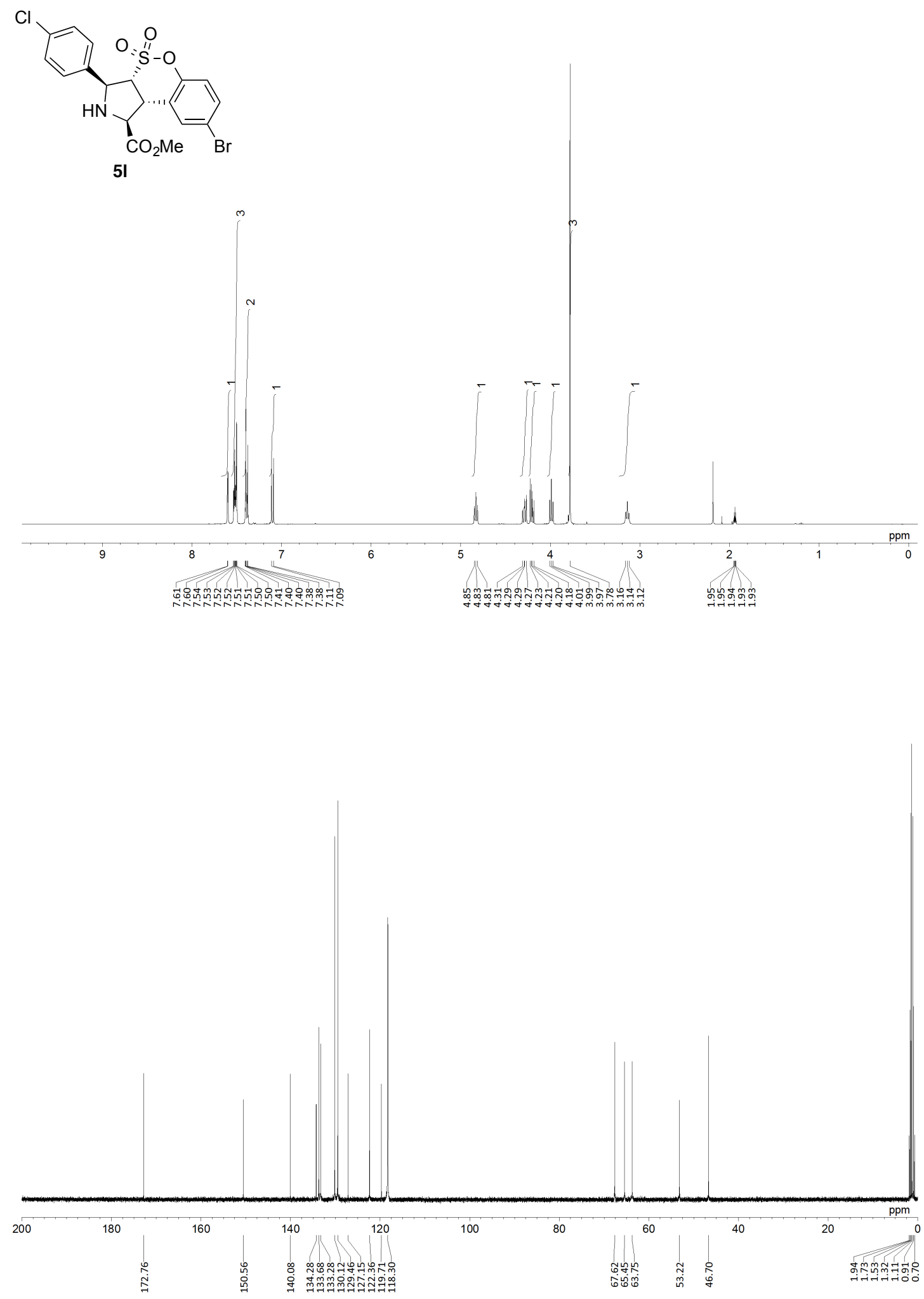


\section{HPLC spectra of Compounds}

HPLC spectra of $\mathbf{4 a}$ and $r a c-4 a$

2 hex70 181 min 1.0m/ id-3 sf-256 20190522_0522 2 hex70 181min 1.0m/ id-3 sf-256 20190522_001 2019/05/22 16:52:00

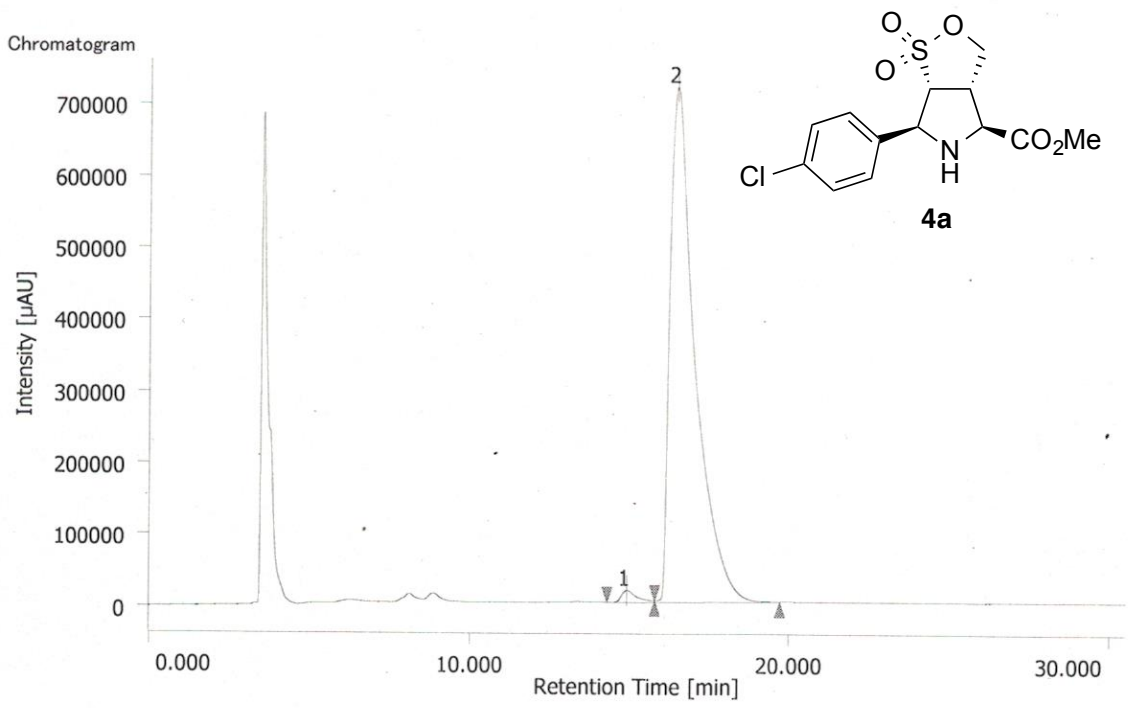

Peak Information

\begin{tabular}{|l|l|l|l|l|l|l|}
\hline \# & Peak Name & tR $[\mathrm{min}]$ & Area $[\mu \mathrm{V} \cdot \mathrm{sec}]$ & Height [uV] & Area\% & Height\% \\
\hline
\end{tabular}

\begin{tabular}{|r|r|r|r|r|r|}
\hline 1 Unknown & 14.927 & 574725 & 16732 & 1.453 & 2.277 \\
\hline 2 Unknown & 16.457 & 38973376 & 718093 & 98.547 & 97.723 \\
\hline
\end{tabular}

2 A70 1.0m/ id-3 rac_p-cl 20190423.04232 A70 1.0m/ id-3 rac_p-cl 20190423_001 2020/03/30 12:21:08

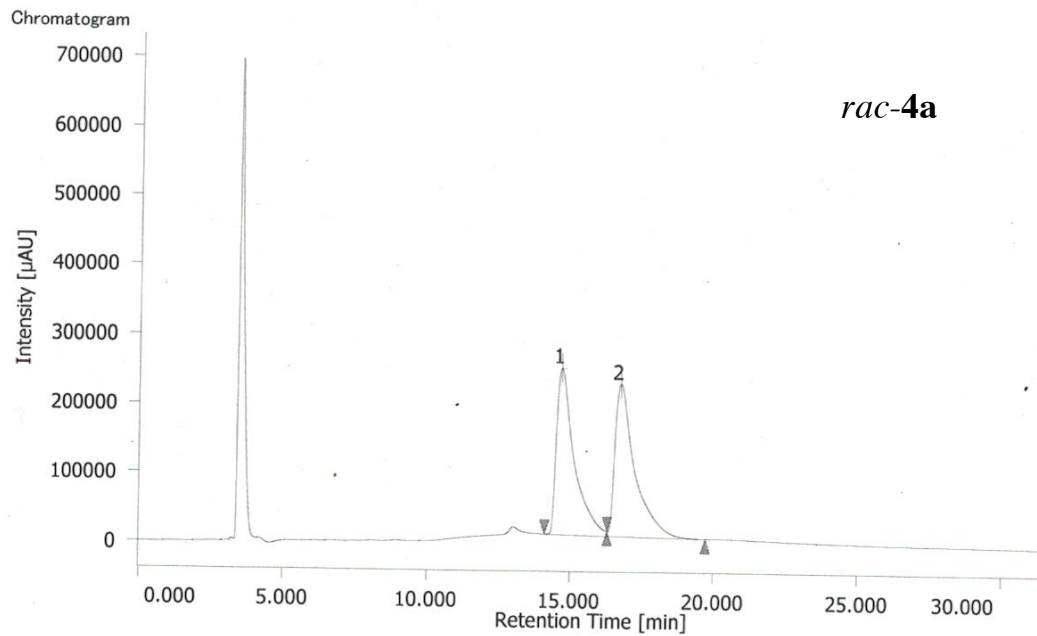

Peak Information

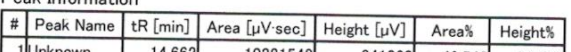

\begin{tabular}{|r|r|r|r|r|r|}
\hline 1 Unknown & 14.662 & 10381549 & 241860 & 49.543 & 52.253 \\
\hline
\end{tabular}

\begin{tabular}{|l|r|r|r|r|r|}
\hline 2 Unknown & 16.735 & 10572955 & 221003 & 50457 & 47747 \\
\hline
\end{tabular}


HPLC spectra of $\mathbf{4 b}$ and $r a c-4 b$

1 hex70 181min 1.0m/ IH-3 sf-281_ph 20190706_0706 1 hex70 181 min 1.0m/ IH-3 sf-281_oh 20190706_001 2019/07/06 21:31:34

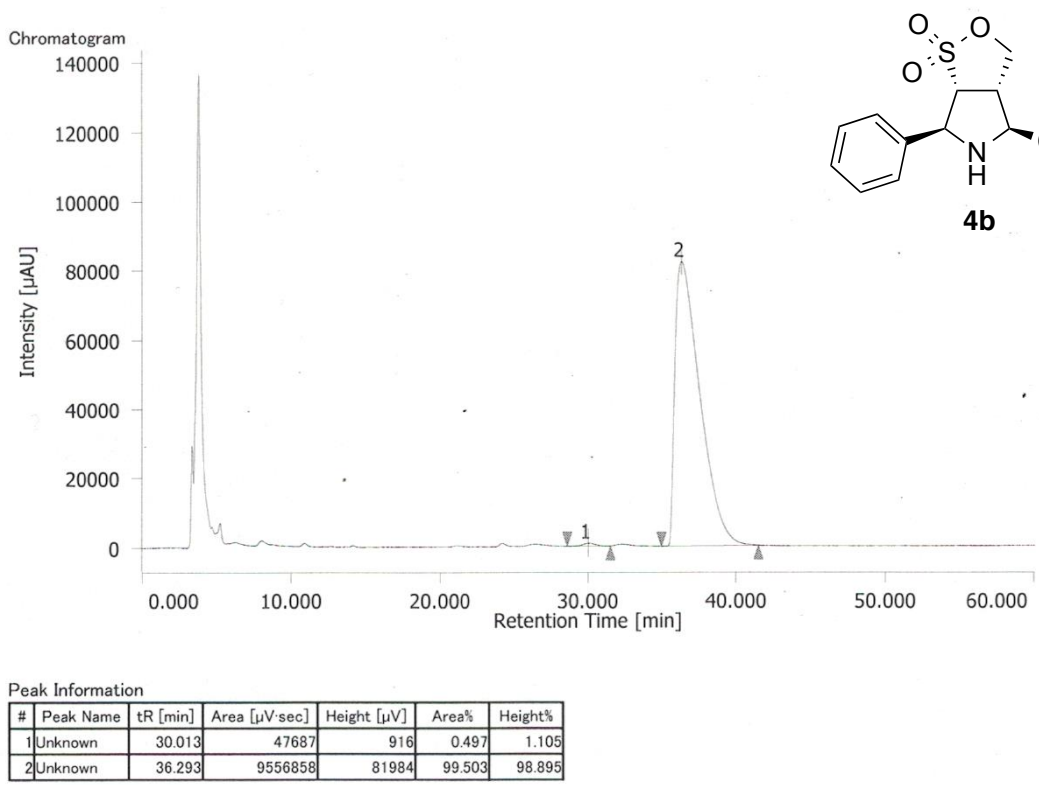

1 hex70 181min 1.0m/ IH-3 sf-rac ph 201907060706 I hex70 181min 1.0m/ IH-3 sf-rac ph 20190706001 2019/07/06 16:32.31

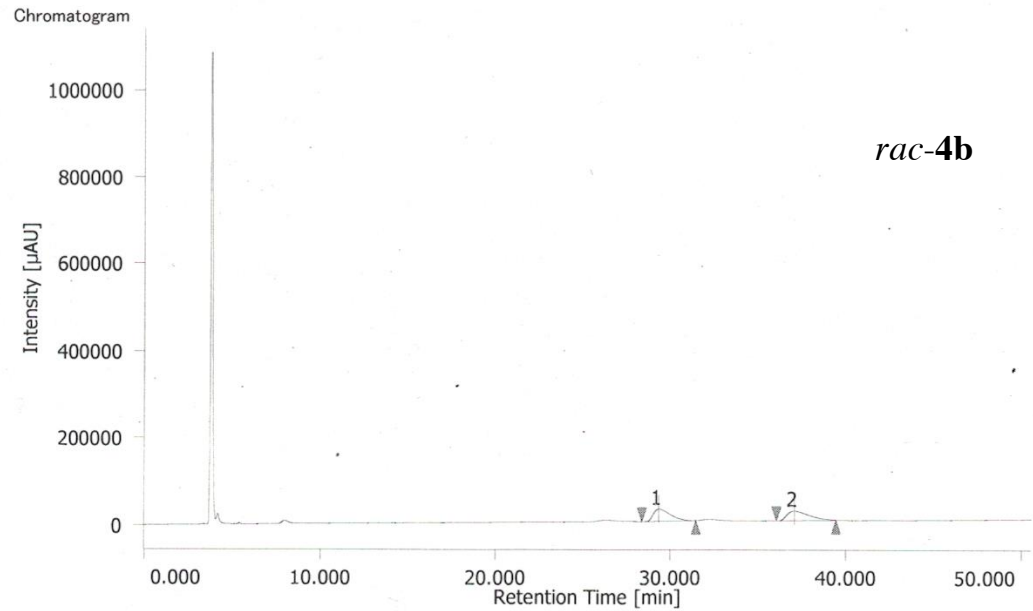

Peak Information

\begin{tabular}{|l|l|l|l|l|l|}
\hline Peak Name & tR [min] & Area $[\mu \mathrm{V} \cdot$ sec $]$ & Height $[\mu \mathrm{V}]$ & Area\% & Height\% \\
\hline & & &
\end{tabular}

\begin{tabular}{|l|r|r|r|r|r|}
\hline 1 Unknown & 29.347 & 1879133 & 27774 & 49.814 & 56.13 \\
\hline 2Unknown & 37.067 & 1893142 & 21707 & 50.186 & 43.869 \\
\hline
\end{tabular} 
HPLC spectra of $\mathbf{4 c}$ and $r a c-4 c$

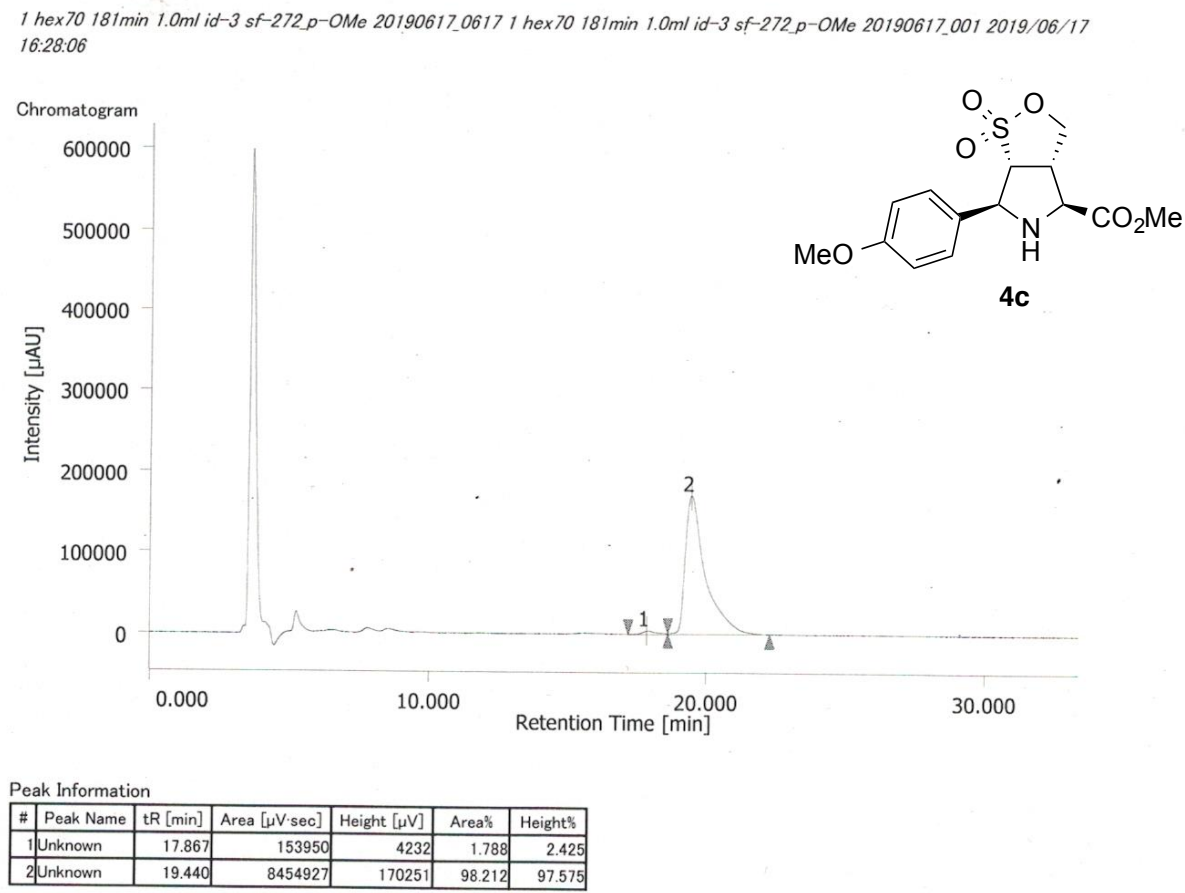

I hex70 181 min 1.0m/ id-3 rac_p-OMe 20190617_06171 hex70 181 min 1.0m/ id-3 rac_p-OMe 20190617_001 2019/06/17 15:56:20

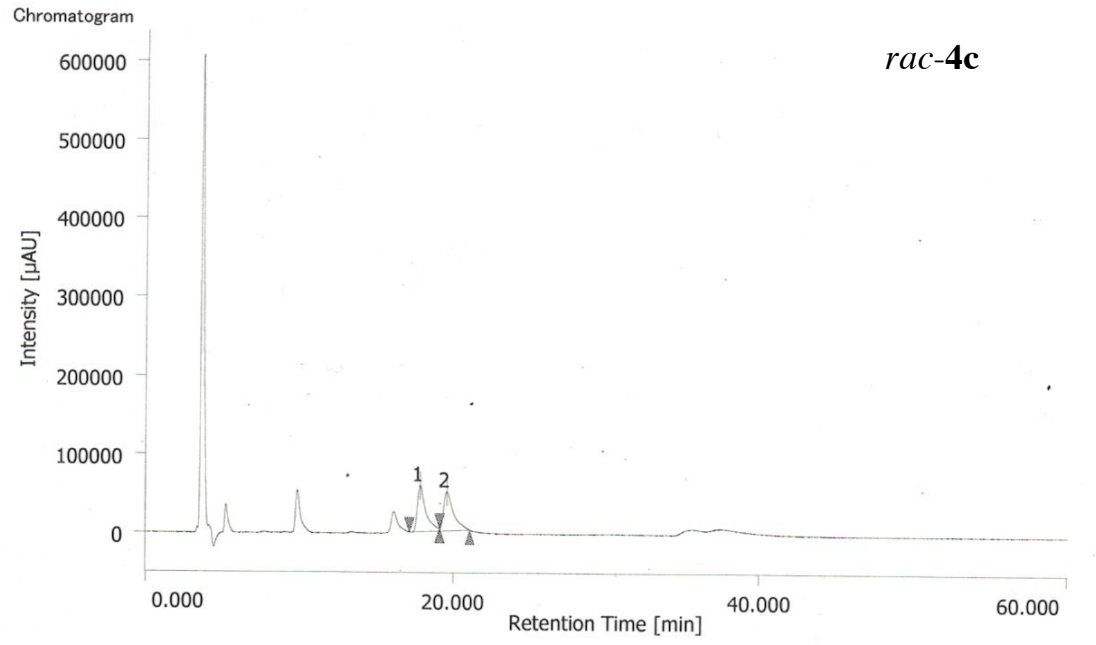

Peak Information

\begin{tabular}{|l|l|l|l|l|l|l|}
$\#$ & Peak Name & tR $[\mathrm{min}]$ & Area $[\mu \mathrm{V} \cdot \mathrm{sec}]$ & Height $[\mu \mathrm{V}]$ & Area\% & Height\% \\
\hline
\end{tabular}

\begin{tabular}{|l|r|r|r|r|r|}
\hline 1 Unknown & 17.840 & 2222964 & 58218 & 50.255 & 54.19 \\
\hline
\end{tabular}


HPLC spectra of $\mathbf{4 d}$ and rac-4d

I hex70 181min 1.0m/ id-3 sf-271_o-Me 20190617_0617 1 hex70 181min 1.0m/ id-3 sf-271_p-Me 20190617001 2019/06/17 13:05:23

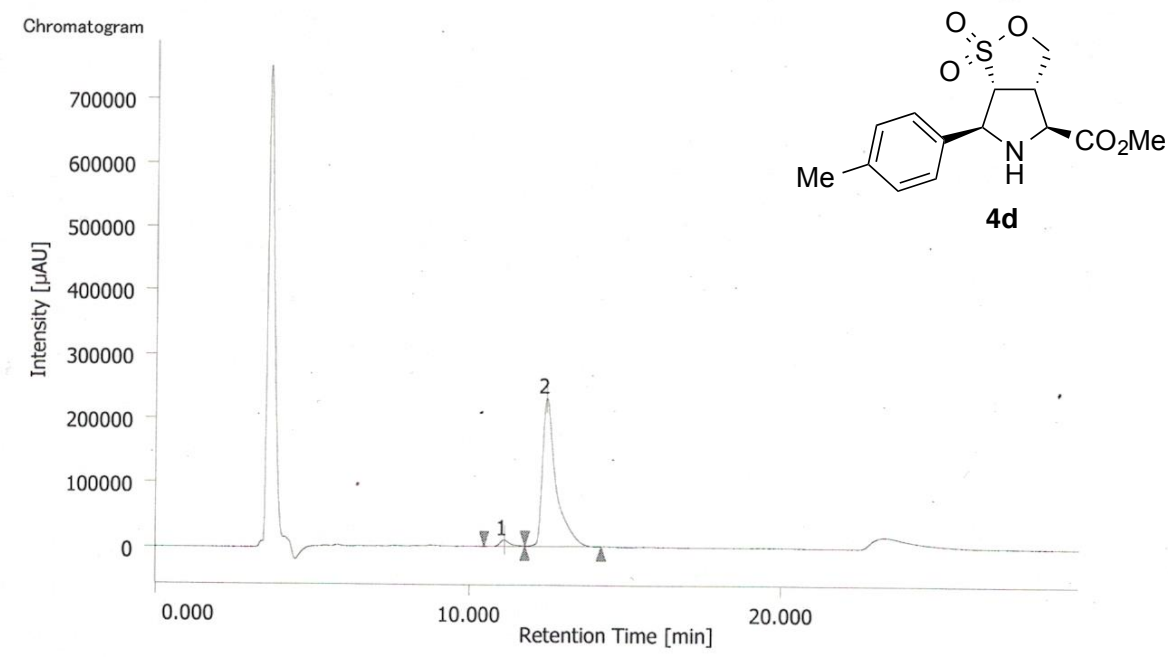

Peak Information

\begin{tabular}{|r|l|r|r|r|r|r|}
\hline$\#$ & Peak Name & tR $[\mathrm{min}]$ & Area $[\mu \mathrm{V} \cdot \mathrm{sec}]$ & Height [uV] & Area\% & Height' \\
\hline 1 & Unknown & 11.133 & 229094 & 9894 & 3220 & 4.126 \\
\hline
\end{tabular}

\begin{tabular}{|l|r|r|r|r|r|}
\hline Unknown & 11.133 & 229094 & 9894 & 3.222 & 4.126 \\
\hline 2 Unknown & 12.467 & 6880150 & 229905 & 96.778 & 95.87 \\
\hline
\end{tabular}

$1 \mathrm{hex} 70181 \mathrm{~min} 1.0 \mathrm{ml}$ id-3 rac_p-Me 20190617_0617 1 hex70 181min 1.0m/ id-3 rac_p-Me 20190617_001 2019/06/17 13:04:18

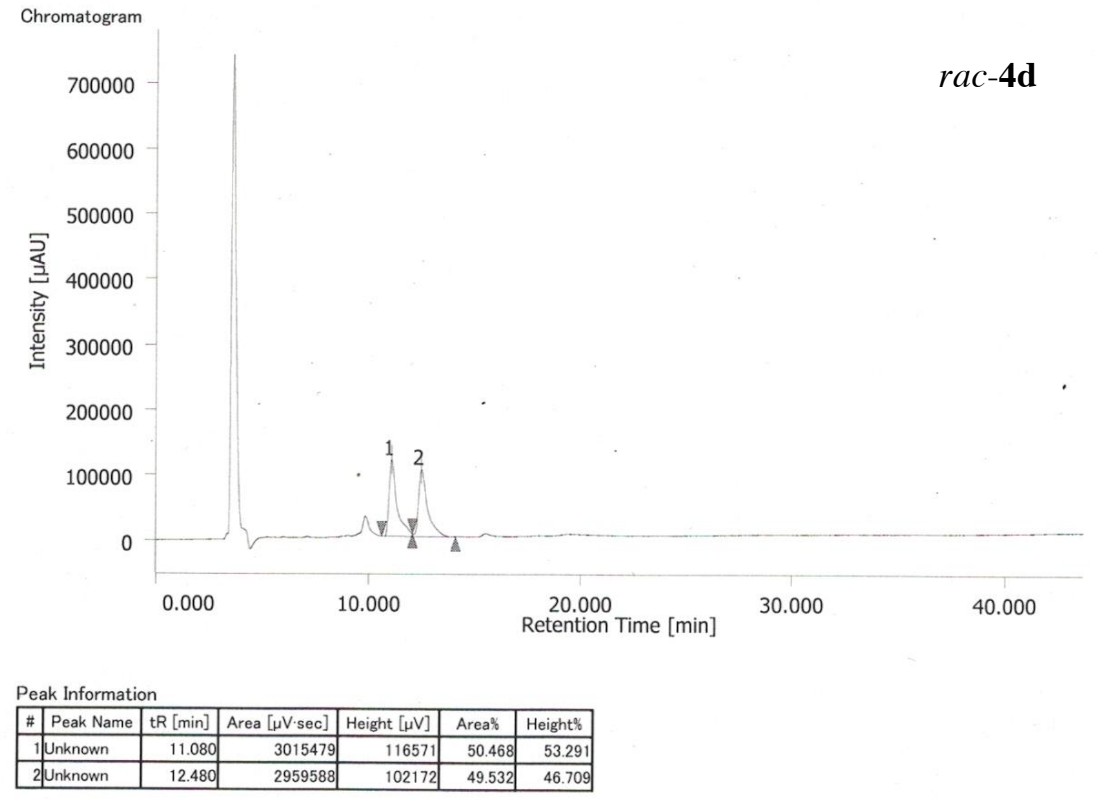


HPLC spectra of $\mathbf{4 e}$ and $r a c-4 e$

1 hex70 181min 1.0m/ id-3 sf-270_p-Br 20190617_0617 1 hex70 181min 1.0m/ id-3 sf-2970_p-Br 20190617_001 2019/06/17 14:59:13

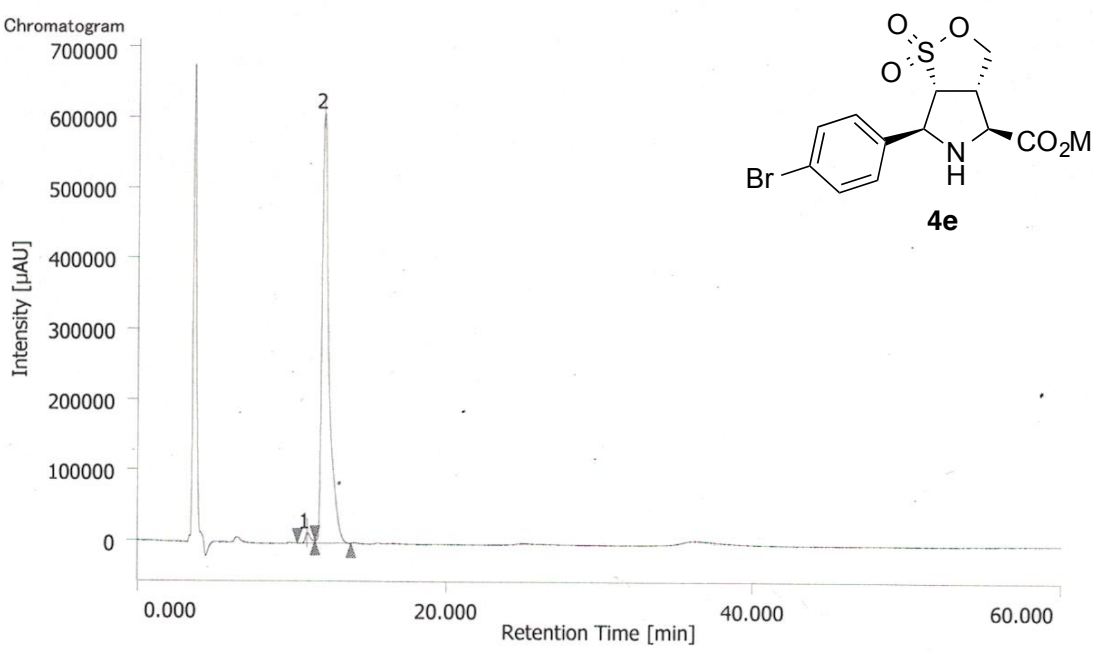

Peak Information

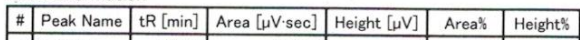

\begin{tabular}{|l|r|r|r|r|r|}
\hline 1 Unknown & 10.987 & 341257 & 14532 & 1.720 & 2.332 \\
\hline 2 Unknown & 12.000 & 19496300 & 608744 & 98.280 & 97.668 \\
\hline
\end{tabular}

\begin{tabular}{l|r|r|}
\hline 2 Unknown & 12.000 & 19496300 \\
\hline
\end{tabular}

I hex70 181 min 1.0m/ id-3 rac_p-Br 20190617 _0617 1 hex70 $181 \mathrm{~min} 1.0 \mathrm{ml}$ id-3 rac_p-Br 20190617_001 2019/06/17 14:58:29

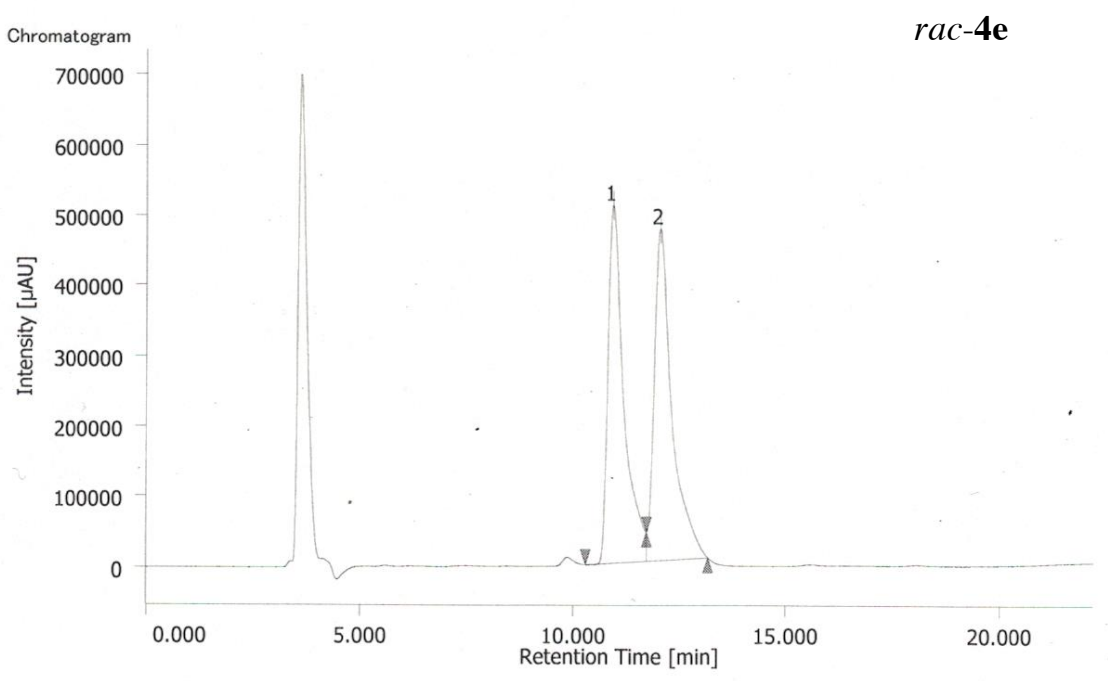

Peak Information

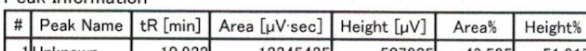

\begin{tabular}{|l|r|r|r|r|r|}
\hline 1 Unknown & 10.933 & 13345435 & 507905 & 49.595 & 51.91 \\
\hline
\end{tabular}


HPLC spectra of $\mathbf{4 f}$ and $r a c-4 f$

1 hex80 181miin 1.0m/ id-3 sf-278_p-F 20190622_0622 1 hex80 181min 1.0m/ id-3 sf-278_p-F 20190622_001 2019/06/22 18:20:36

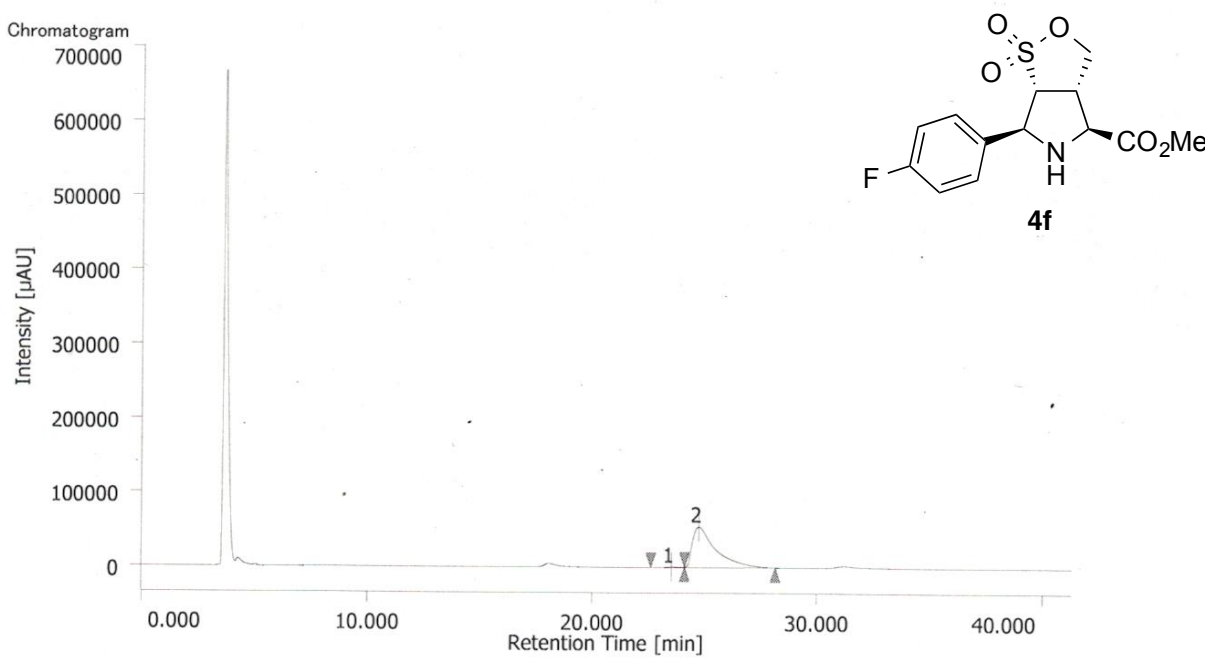

Peak Information

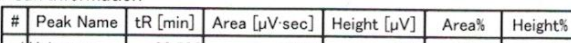

\begin{tabular}{|l|r|r|r|r|r|}
\hline U Unknown & 23.533 & 29397 & 716 & 0.742 & 1.297 \\
\hline
\end{tabular}

\begin{tabular}{|l|r|r|r|r|r|}
\hline 2Unknown & 24.747 & 3934503 & 54443 & 99.258 & 98.703 \\
\hline
\end{tabular}

1 hex80 181miin 1.0m/ id-3 rac_p-F_2 20190622_0622 t hex80 181 miin 1.0m/ id-3 rac_-_-F_2 20190622.001 2019/06/22 17: rac-4f

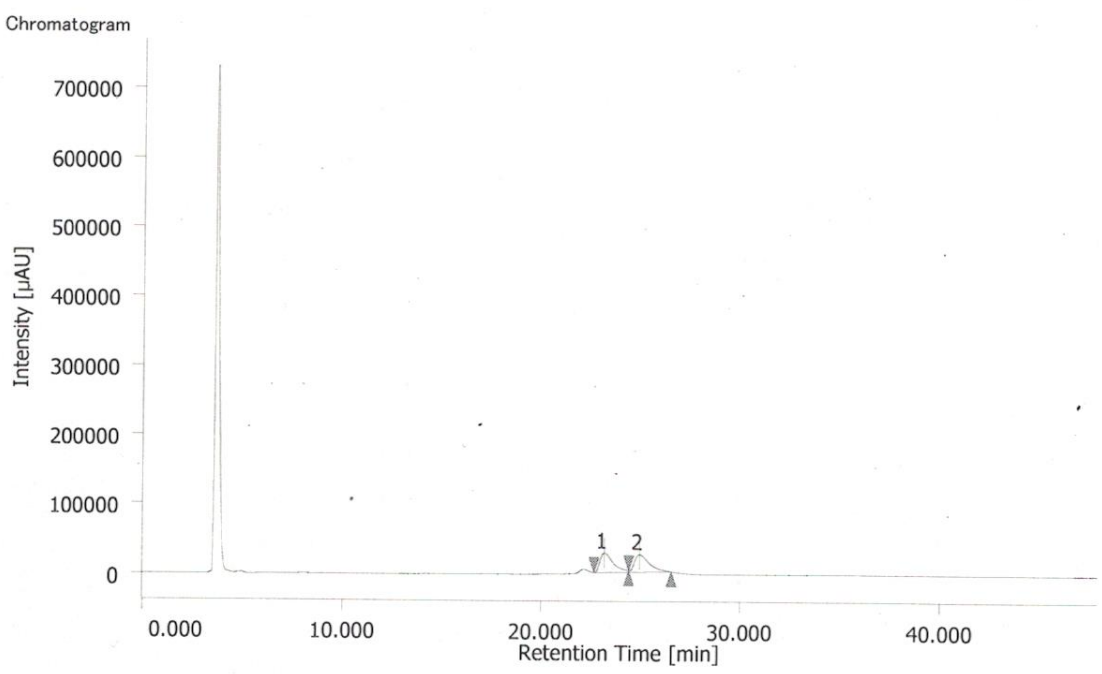

Peak Information

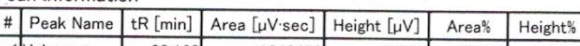

\begin{tabular}{|l|r|r|r|r|r|}
\hline 1 Unknown & 23.160 & 1312458 & 27871 & 49.944 & 52.73 \\
\hline 2Unknown & 24.960 & 1315407 & 24977 & 50.056 & 47.26 \\
\hline
\end{tabular}


HPLC spectra of $\mathbf{4 g}$ and $r a c-4 g$

1 hex70 181min 1.0m/ id-3 sf-267p-cf3 20190605.06051 hex70 $181 \mathrm{~min} 1.0 \mathrm{~m} / \mathrm{id}-3 \mathrm{sf}-267$ p-cf3 20190605 001 2019/06/05 14:02:07

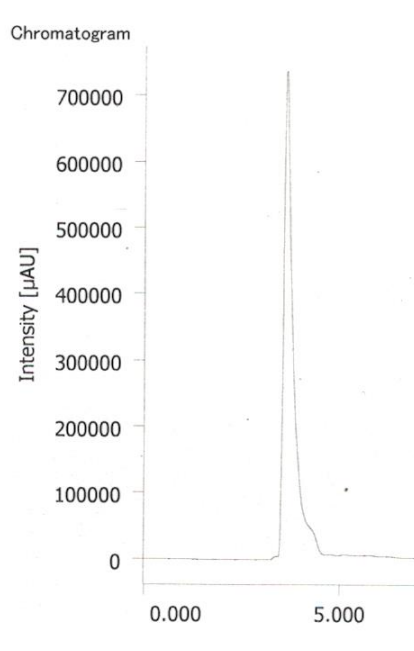

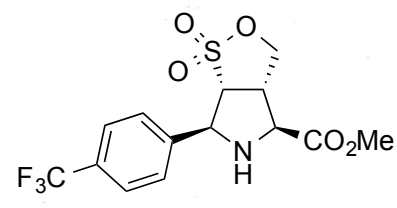

$4 \mathrm{~g}$

Peak Information

\begin{tabular}{|l|l|l|l|l|l|l|}
\hline Peak Name & tR $[\mathrm{min}]$ & Area $[\mu \mathrm{V} \cdot \mathrm{sec}]$ & Height $[\mu \mathrm{V}]$ & Area\% & Height\% \\
\hline
\end{tabular}

\begin{tabular}{|l|r|r|r|r|r|}
\hline 1 Unknown & 7.693 & 196420 & 9701 & 2.339 & 2.83 \\
\hline 2Unknown & 8.467 & 8199815 & 332548 & 97.661 & 97.165 \\
\hline
\end{tabular}

1 hex70 181 min 1.0m/ id -3 rac_p-cf3 20190605_0605 I hex70 181 min 1.0m/ id-3 rac_p-cf3 20190605_001 2019/06/05 13:37:32

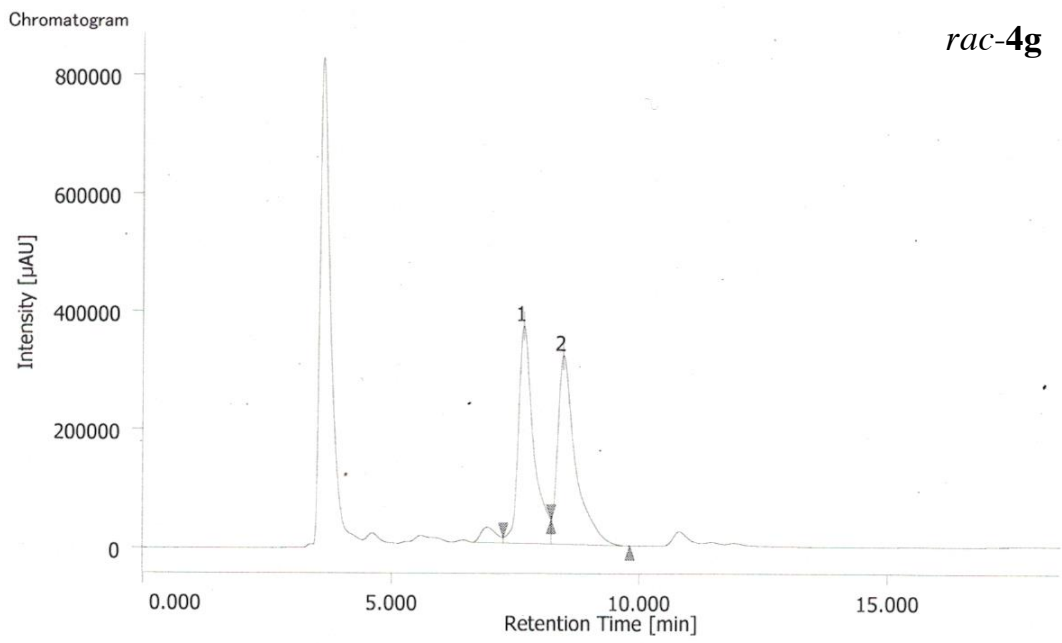

Peak Information

\begin{tabular}{|l|l|l|l|l|l|l|}
\hline Peak Name & tR $[\mathrm{min}]$ & Area $[\mu \mathrm{V} \cdot \mathrm{sec}]$ & Height $[\mu \mathrm{V}]$ & Area\% & Height\% \\
\hline
\end{tabular}

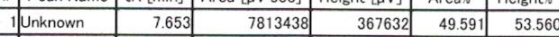

\begin{tabular}{|l|r|r|r|r|r|}
\hline J Unknown & 7.653 & 7813438 & 367632 & 49.591 & 53.560 \\
\hline
\end{tabular}

\begin{tabular}{l|l|l|l|l|l}
318756 & 50.409 & 46.440 \\
\hline
\end{tabular}


HPLC spectra of $\mathbf{4 h}$ and $r a c-4 h$

1 hex70 181 min 1.0m/ ih-3 sf-467 m-Me_0203 1 hex70 181 min 1.0m/ ih-3 sf-467 m-Me_001 2020/02/03 20:40:33

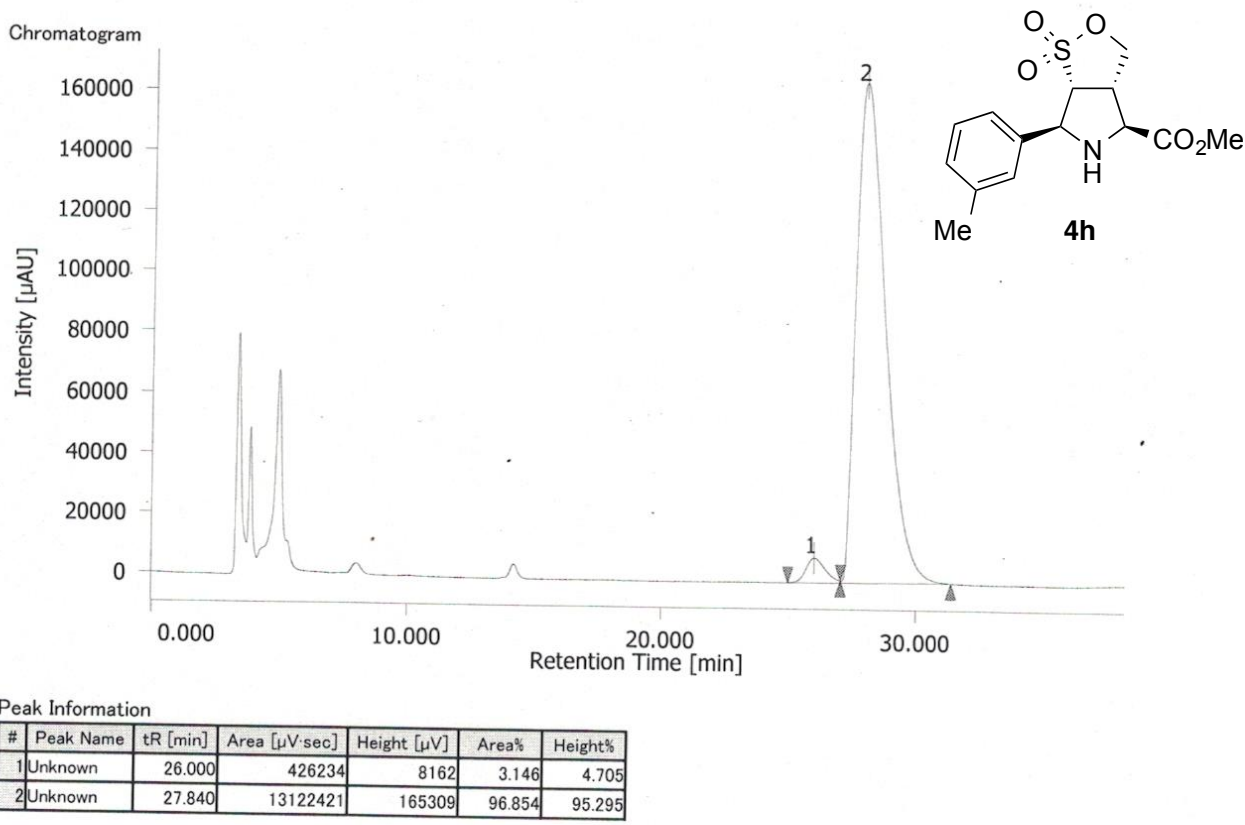

1 hex70 181min 1.0m/ IH-3 sf-rac_m-Me 20190706_0706 1 hex70 181min 1.0m/ IH-3 sfrac_m-Me 20190706_001 2019/07/06 19:30:05

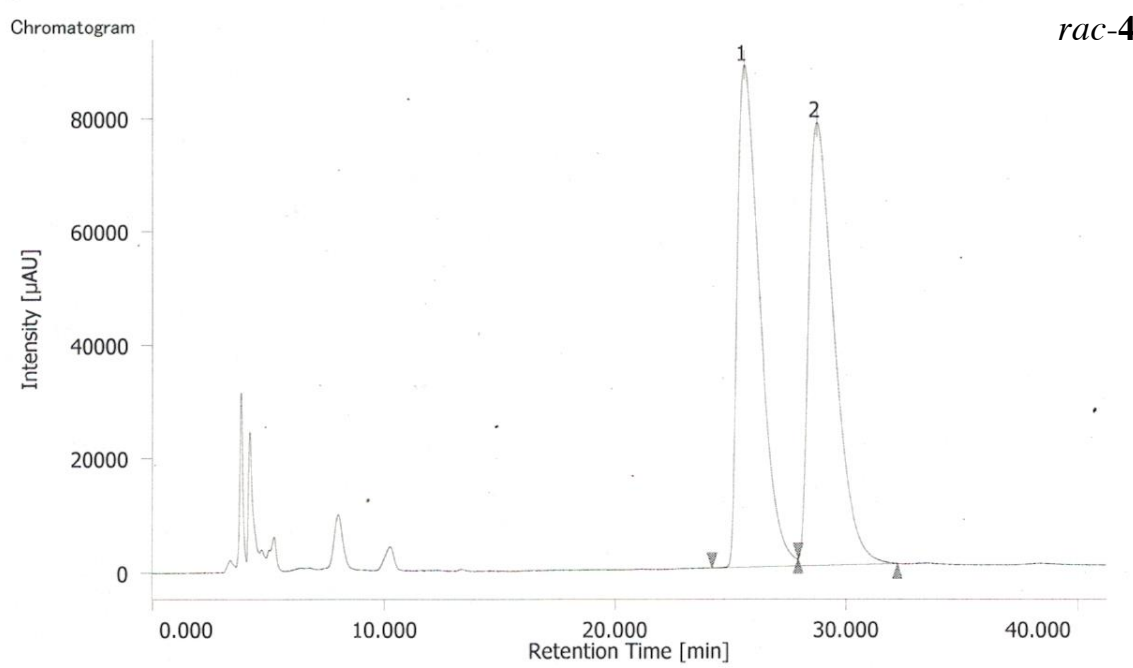

Peak Information

\begin{tabular}{|l|l|r|r|r|r|r|}
\hline$\#$ & Peak Name & tR $[\mathrm{min}]$ & Area $[\mu \mathrm{V} \cdot \mathrm{sec}]$ & Height $[\mu \mathrm{V}]$ & \multicolumn{1}{|c|}{ Area\% } & Height\% \\
\hline 1 & Unknown & 25.573 & 5945767 & 88272 & 49.937 & 53.109 \\
\hline
\end{tabular}

\begin{tabular}{|l|r|r|r|r|r|}
\hline 1 Unknown & 25.573 & 5945767 & 88272 & 49.937 & 53.109 \\
\hline
\end{tabular}

\begin{tabular}{l|r|r|r|r|r|}
\hline 2 Unknown & 28.720 & 5960667 & 77938 & 50.063 & 46.891 \\
\hline
\end{tabular}


HPLC spectra of $\mathbf{4 i}$ and $r a c-4 \mathbf{i}$

I hex70 181min 1.0m/ id-3 sf-273_m-F 20190624_0624 t hex70 181 min 1.0m/ id-3 sf-273_m-F 20190624_001 2019/06/24 20:12:22

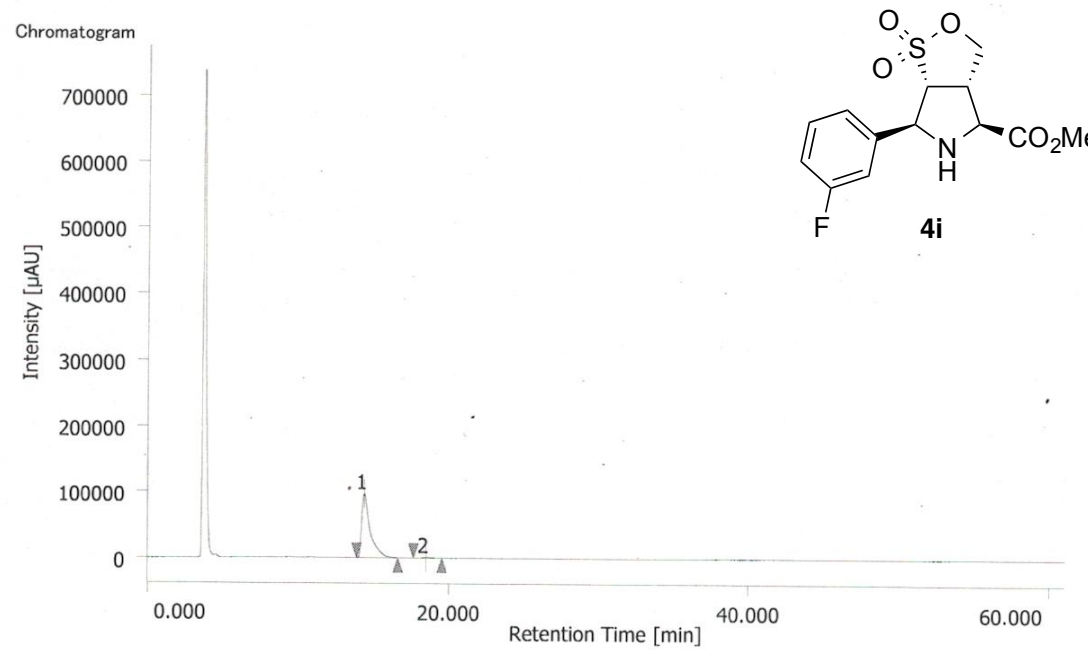

Peak Information

\begin{tabular}{|l|l|l|l|l|l|}
\hline Peak Name & tR $[\mathrm{min}]$ & Area $[\mu \mathrm{V} \cdot \mathrm{sec}]$ & Height $[\mu \mathrm{V}]$ & Area\% & Height\% \\
\hline
\end{tabular}

\begin{tabular}{|l|r|r|r|r|r|}
\hline 1 Unknown & 14.373 & 3734053 & 96441 & 98.829 & 98.786 \\
\hline
\end{tabular}

\begin{tabular}{|l|r|r|r|r|r|}
\hline 2 U Unknown & 18.480 & 44243 & 1185 & 1.171 & 1.214 \\
\hline
\end{tabular}

1 hex70 181min 1.0m/ id-3 rac_m-F 20190624_0624 I hex70 181 min 1.0ml id-3 rac_m-F 20190624_001 2019/06/24 15:29:22

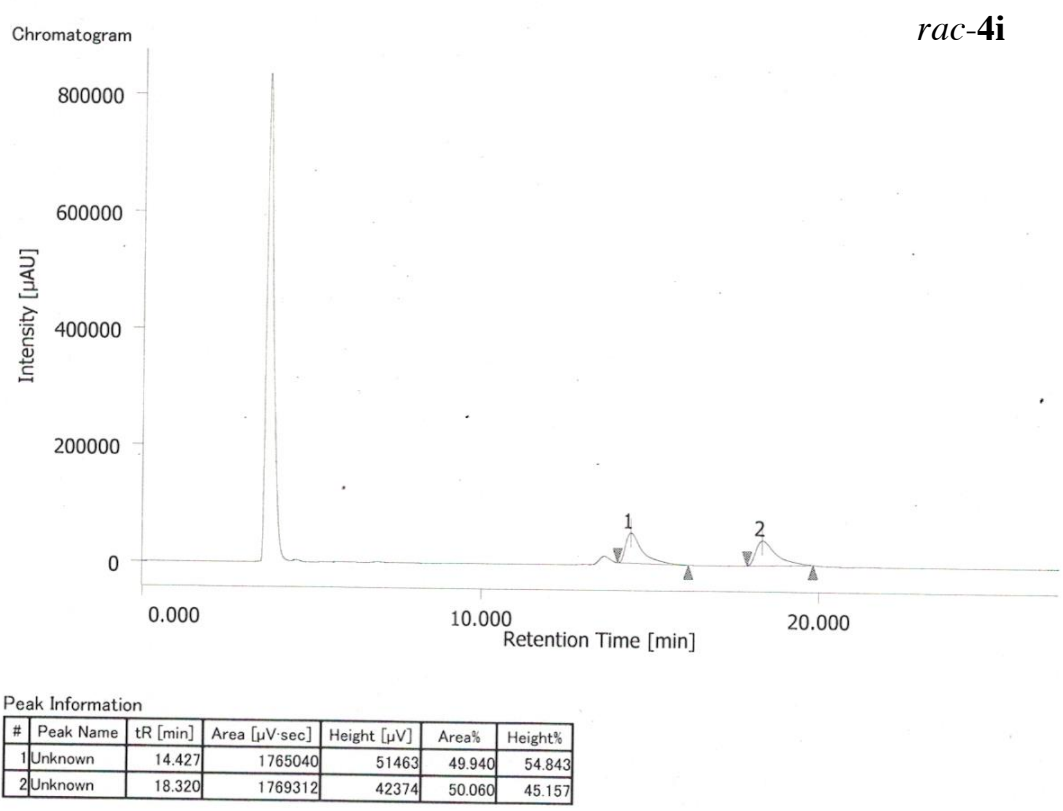


HPLC spectra of $\mathbf{4} \mathbf{j}$ and $r a c-\mathbf{4} \mathbf{j}$

1 hex70 $181 \mathrm{~min} 1.0 \mathrm{ml}$ id-3 sf-266-o-Br-2 20190605_0605 t hex70 $181 \mathrm{~min} 1.0 \mathrm{~m} / \mathrm{id}-3 \mathrm{sf}-266_{-} \mathrm{o}-\mathrm{Br}_{-} 2$ 20190605_001 2019/06/26 13:10:11

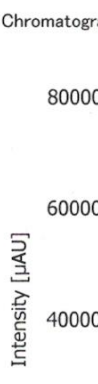

200000

0
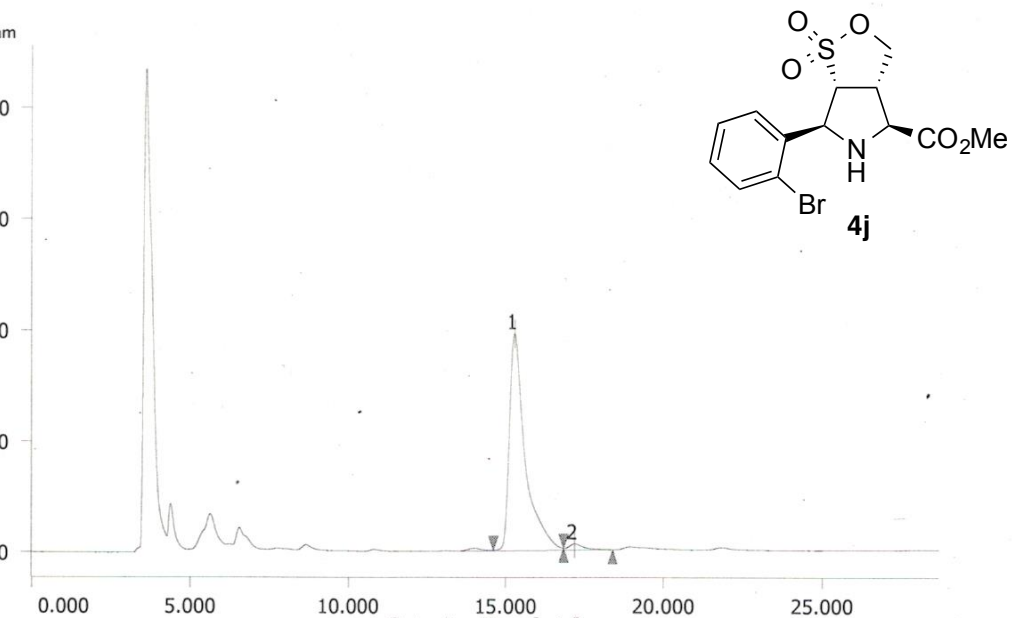

Peak Information

\# Peak Name $\mid$\begin{tabular}{l|r|r|r|r|} 
tR [min] & Area $[\mu \mathrm{V} \cdot \mathrm{sec}]$ & Height $[\mu \mathrm{V}]$ & Area\% & Height\% \\
\hline
\end{tabular}

\begin{tabular}{|l|r|r|r|r|r|}
\hline 1 Unknown & 15.280 & 13571612 & 390998 & 97.306 & 97.15 \\
\hline
\end{tabular}

\begin{tabular}{|l|r|r|r|r|r|r|}
\hline 2 Unknown & 17.173 & 375738 & 11450 & 2.694 & 2.84 \\
\hline
\end{tabular}

1 hex70 181min 1.0m/ id-3 rac_o-Br 20190605,06051 hex70 $181 \mathrm{~min} 1.0 \mathrm{ml}$ id-3 rac_o-Br 20190605_001 2019/06/05 12:31:47

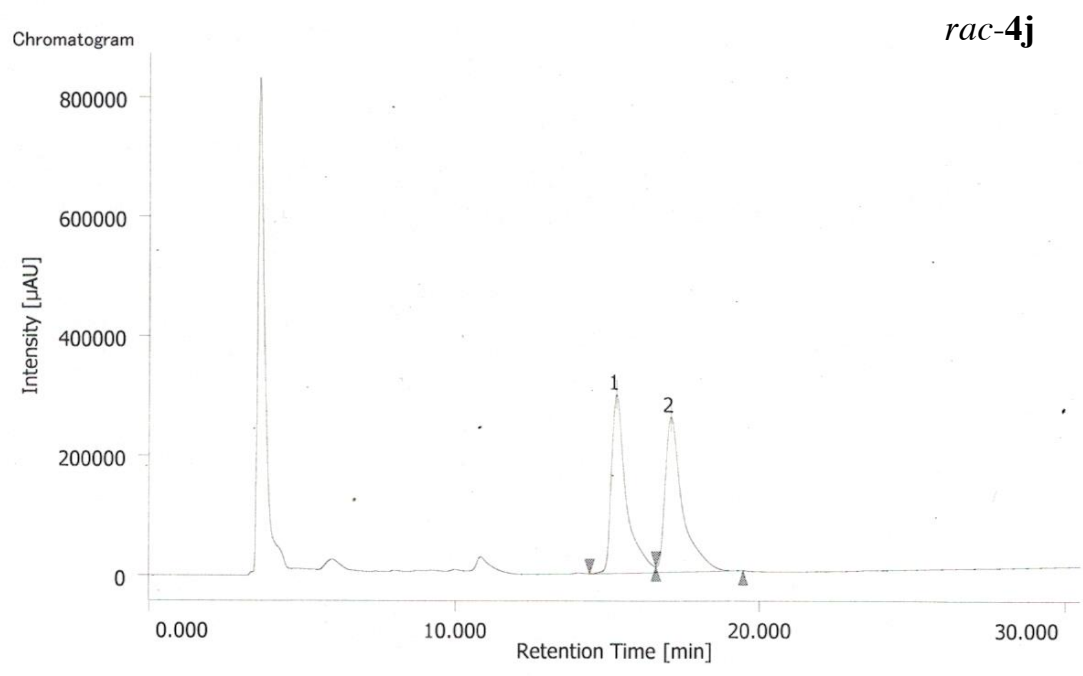

Peak Information

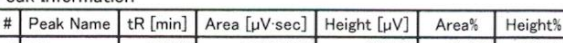

\begin{tabular}{|l|r|r|r|r|r|}
\hline 1 Unknown & 15.293 & 10191802 & 298701 & 49.865 & 53.613 \\
\hline 2Unknown & 17.080 & 10247119 & 258440 & 50.135 & 46.38 \\
\hline
\end{tabular}


HPLC spectra of $\mathbf{4 k}$ and $r a c-4 k$

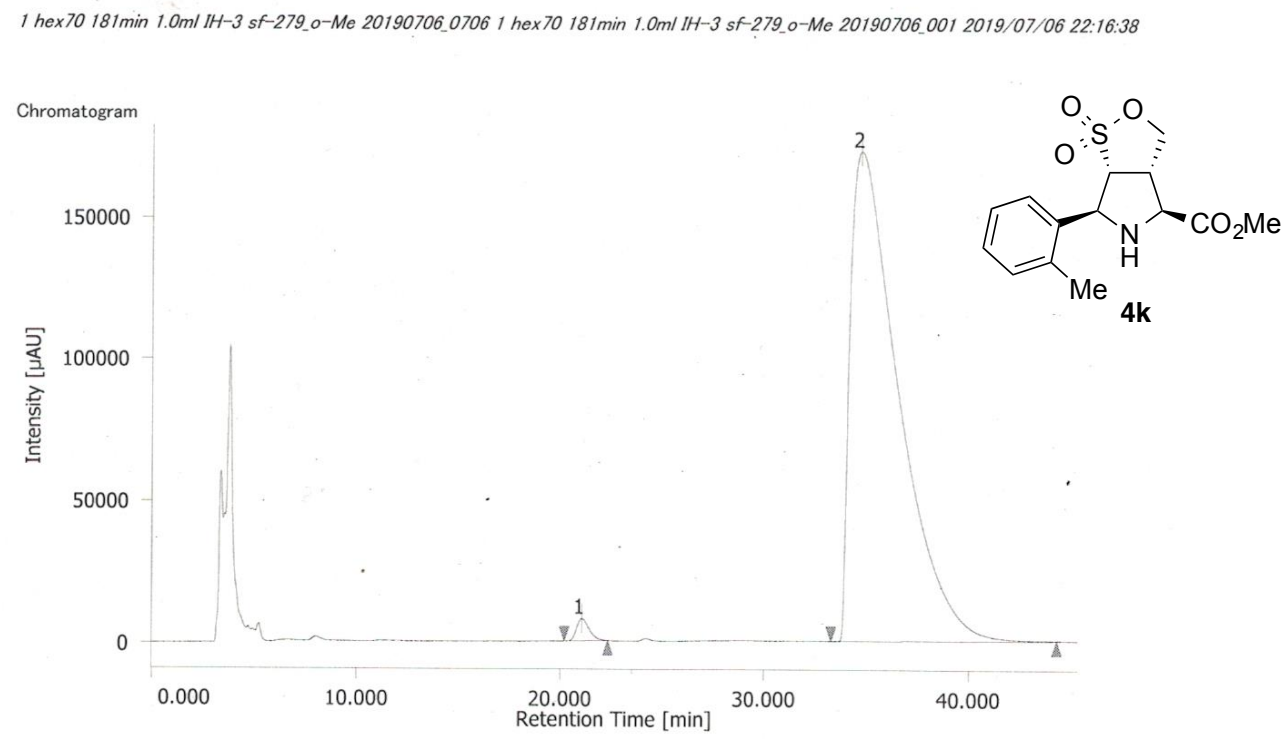

Peak Information

\begin{tabular}{|l|l|r|r|r|r|r|}
\hline$\#$ & Peak Name & tR $[\mathrm{min}]$ & Area $[\mu \mathrm{V} \cdot \mathrm{sec}]$ & Height $[\mu \mathrm{V}]$ & Area\% & Height: \\
\hline
\end{tabular}

\begin{tabular}{|l|r|r|r|r|r|}
\hline Uunknown & 21.040 & 334173 & 7825 & 1.171 & 4.350 \\
\hline 2 Unknown & 34.667 & 28205487 & 172046 & 98.829 & 95.650 \\
\hline
\end{tabular}

1 hex70 $181 \mathrm{~min} 1.0 \mathrm{ml}$ IH-3 sf-rac_o-Me_2 20190706_0706 I hex70 $181 \mathrm{~min} 1.0 \mathrm{ml} / \mathrm{HH}-3$ sf-rac_o-Me_2 20190706_001 2019/07/06 18:44:57

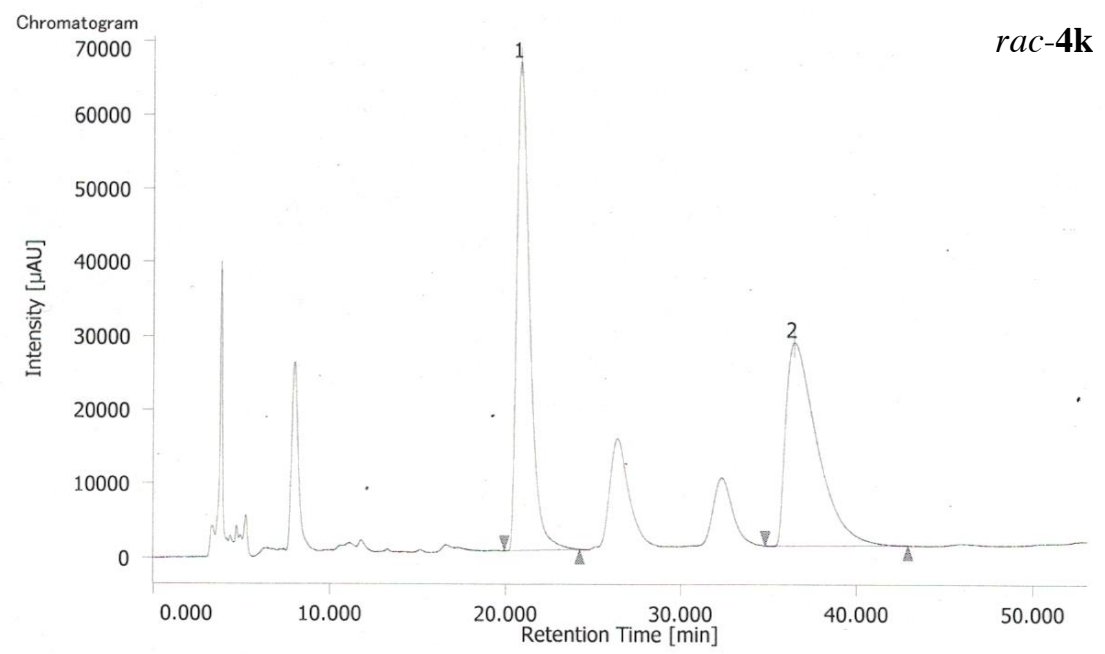

Peak Information

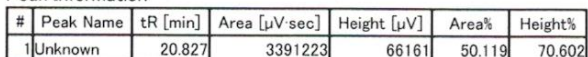

\begin{tabular}{|c|c|c|c|c|c|}
\hline Unknown & \begin{tabular}{l|}
20.827 \\
\end{tabular} & 3391223 & 66161 & 50.119 & 70.602 \\
\hline 2 Unknown & 36.413 & 3375179 & 27549 & 49.881 & 29.398 \\
\hline
\end{tabular}


HPLC spectra of $\mathbf{4 l}$ and $r a c-41$

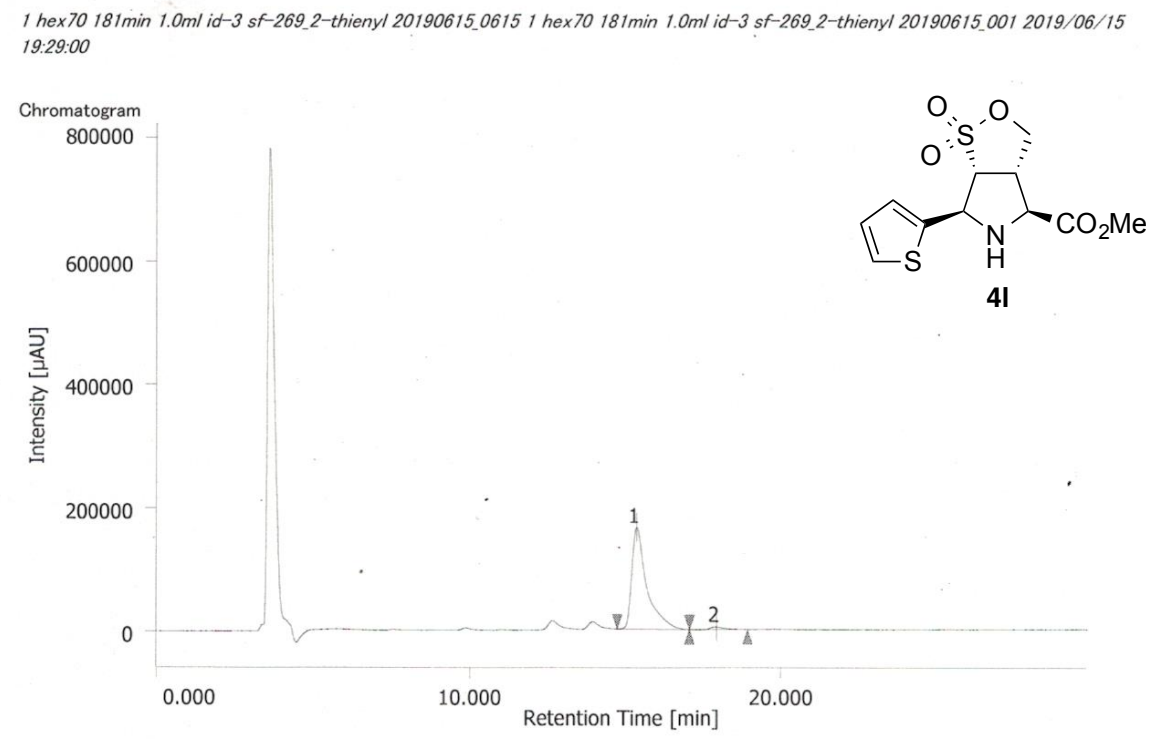

Peak Information

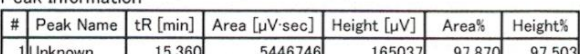

\begin{tabular}{|l|r|r|r|r|r|}
\hline 1 Unknown & 15.360 & 5446746 & 165037 & 97.870 & 97.503 \\
\hline 2 Unknown & 17.920 & 118536 & 4226 & 2130 & 2497 \\
\hline
\end{tabular}

1 hex70 181min 1.0m/ id-3 rac_2-thienyl 20190615_06151 hex70 $181 \mathrm{~min} 1.0 \mathrm{ml} / \mathrm{id}^{-3} \mathrm{rac}$ 2-thienyl 20190615_001 2019/06/15 19:37:21

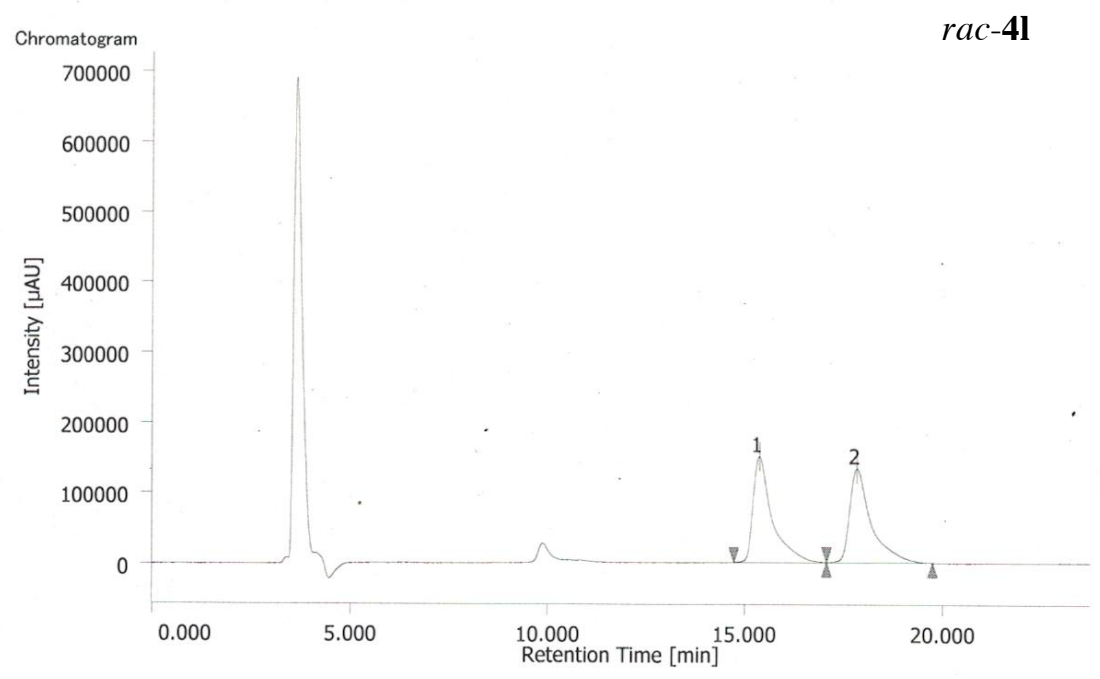

Peak Information

\begin{tabular}{|l|l|r|r|r|r|r|}
\hline$\#$ & Peak Name & tR $[\mathrm{min}]$ & Area $[\mu \mathrm{V} \cdot \mathrm{sec}]$ & Height $[\mu \mathrm{V}]$ & Area\% & Height\% \\
\hline 1 Unknown & 15.373 & 4993528 & 149630 & 50.168 & 53032 \\
\hline
\end{tabular}

\begin{tabular}{|l|r|r|r|r|r|}
\hline 1 Unknown & 15.373 & 4993528 & 149630 & 50.168 & 53.032 \\
\hline
\end{tabular}


HPLC spectra of $\mathbf{5 a}$ and $r a c-5 a$

1 hex70 181min 1.0m/ id-3 sf-400 20191105.1105 1 hex70 181min 1.0m/ id-3 sf-400 20191105_001 2020/03/17 14:35:46

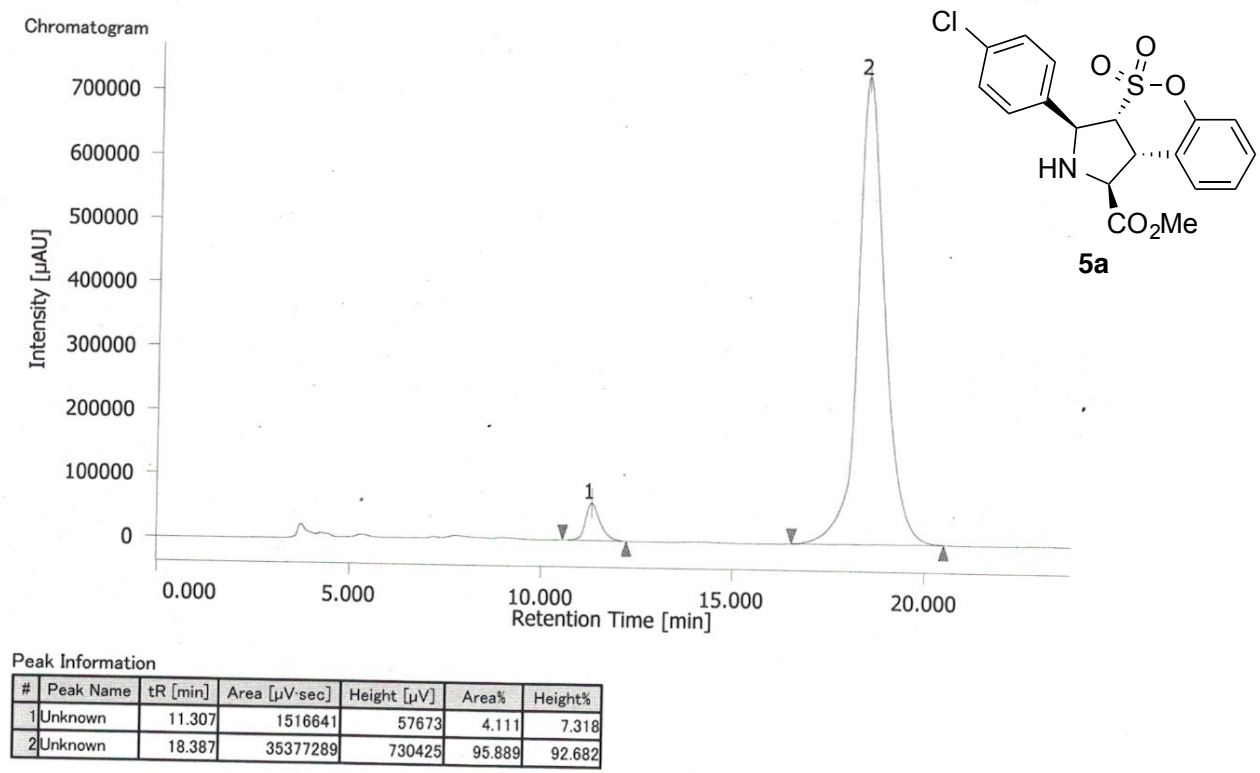

1 hex70 181min 1.0m/ id-3 sf-249_rac 20190622_06221 hex70 181 miin 1.0m/ id-3 sf-249_rac 20190622.001 2019/06/22 14:45:01

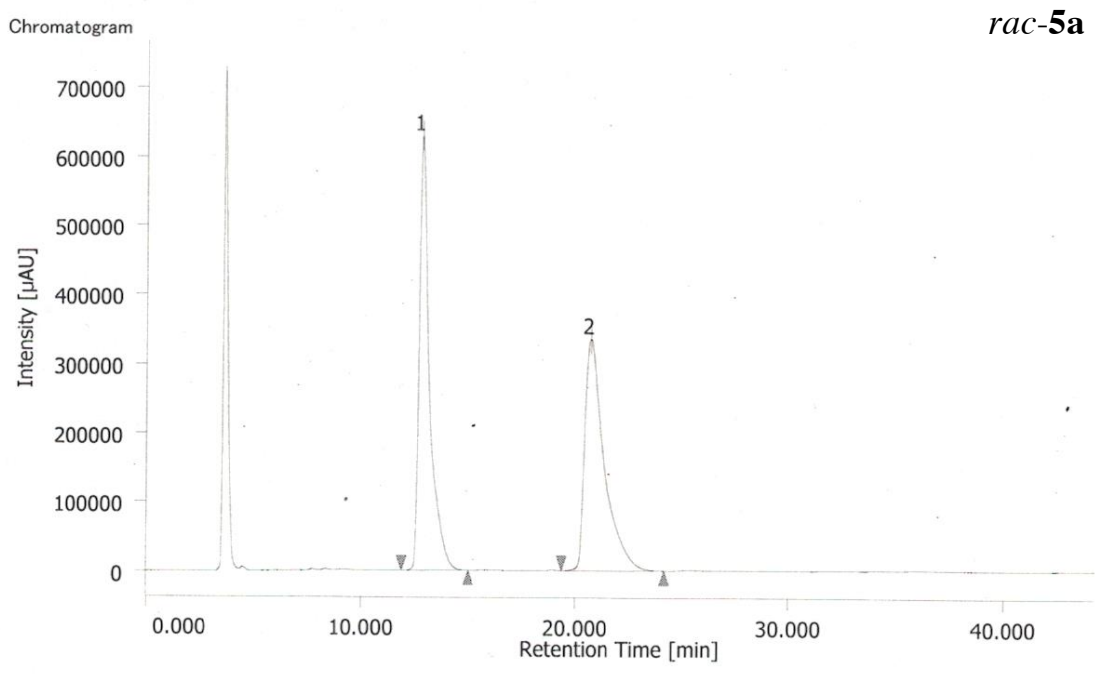

Peak Information

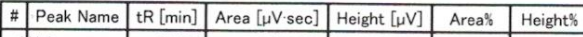

\begin{tabular}{|l|r|r|r|r|r|}
\hline 1 Unknown & 12.800 & 21077488 & 629473 & 49.746 & 65.27 \\
\hline
\end{tabular}

\begin{tabular}{|l|r|r|r|r|r|}
\hline 2 Unknown & 20.707 & 21293089 & 334805 & 50.254 & 34.721 \\
\hline
\end{tabular} 
HPLC spectra of $\mathbf{5 b}$ and $r a c-\mathbf{5 b}$

1 hex70181min 1.0ml id-3 sf-436 chiral_ph 20191204_1204 I hex70 181min 1.0m/ id-3 sf-436 chiral_ph 20191204_001 2019/12/21 14:41:19

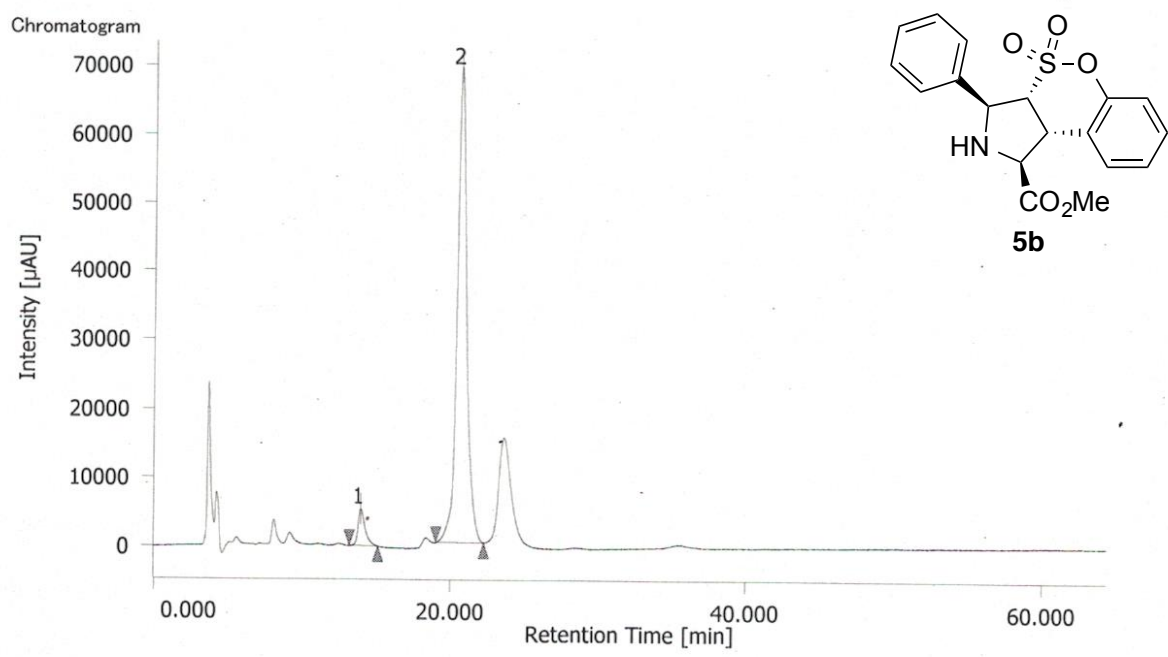

Peak Information

\begin{tabular}{|l|l|r|r|r|r|r|}
\hline$\#$ & Peak Name & tR $[\mathrm{min}]$ & Area $[\mu \mathrm{V} \cdot \mathrm{sec}]$ & Height $[\mu \mathrm{V}]$ & Area\% & Height\% \\
\hline
\end{tabular}

\begin{tabular}{|r|r|r|r|r|r|}
\hline I Unknown & 13.960 & 178043 & 5369 & 5.462 & 7.197 \\
\hline
\end{tabular}

\begin{tabular}{|l|r|r|r|r|r|}
\hline 2 Unknown & 20.573 & 3081543 & 69235 & 94.538 & 92.803 \\
\hline
\end{tabular}

1 hex70 181min 1.0m/ id-3 sf-435 rac_ph 20191204_12041 hex70 181min 1.0m/ id-3 sf-435 rac_ph 20191204_001 2019/12/21 14:40:48

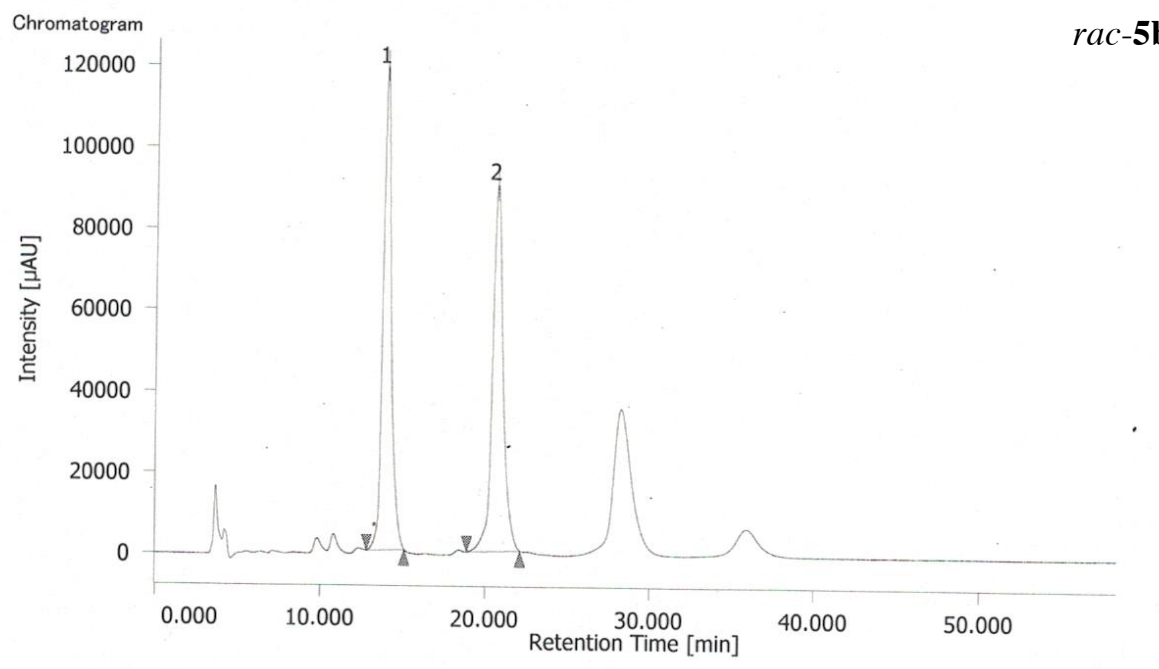

Peak Information

\begin{tabular}{|l|r|r|r|r|r|r|}
\hline Peak Name & tR [min] & Area [ $\mu \mathrm{V} \cdot \mathrm{sec}]$ & Height [uV] & Area\% & Height\% \\
\hline
\end{tabular}

\begin{tabular}{|c|c|c|c|c|c|}
\hline Unknown & 13.853 & 4134325 & 119077 & 50.401 & 56.908 \\
\hline 2 Unknown & 20.587 & 4068533 & 90168 & 49.599 & 43.092 \\
\hline
\end{tabular}


HPLC spectra of $\mathbf{5 c}$ and $r a c-5 c$

1 hex70 181min 1.0m/ id-3 sf-498 p-OMe_02031 hex70 181min 1.0m/ id-3 sf-498 p-OMe_001 2020/02/03 19:28:45

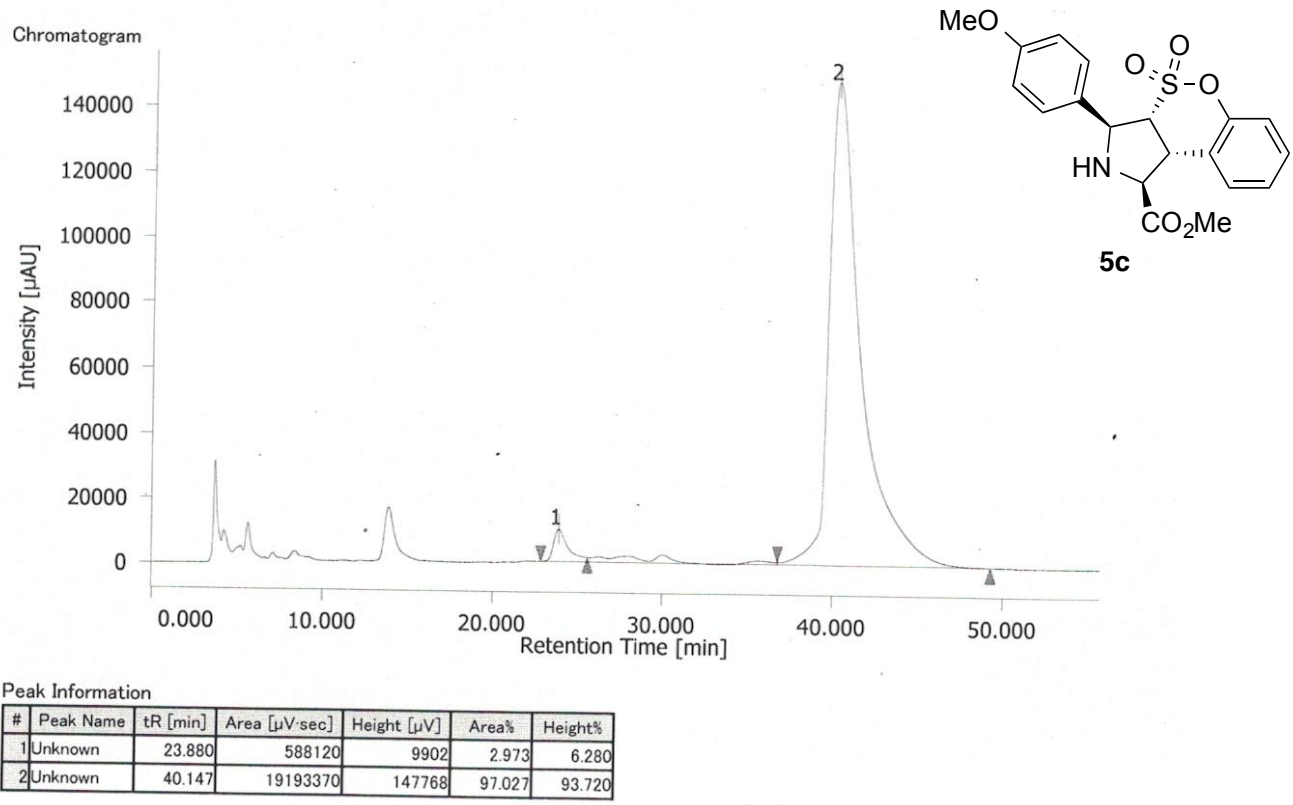

1 hex70 181 min 1.0m/ id-3 sf-432 rac_p-OMe 20191204_12041 hex70 181min 1.0m/ id-3 sf-432 rac_p-OMe 20191204_001 2019/12/21 $14: 39: 43$

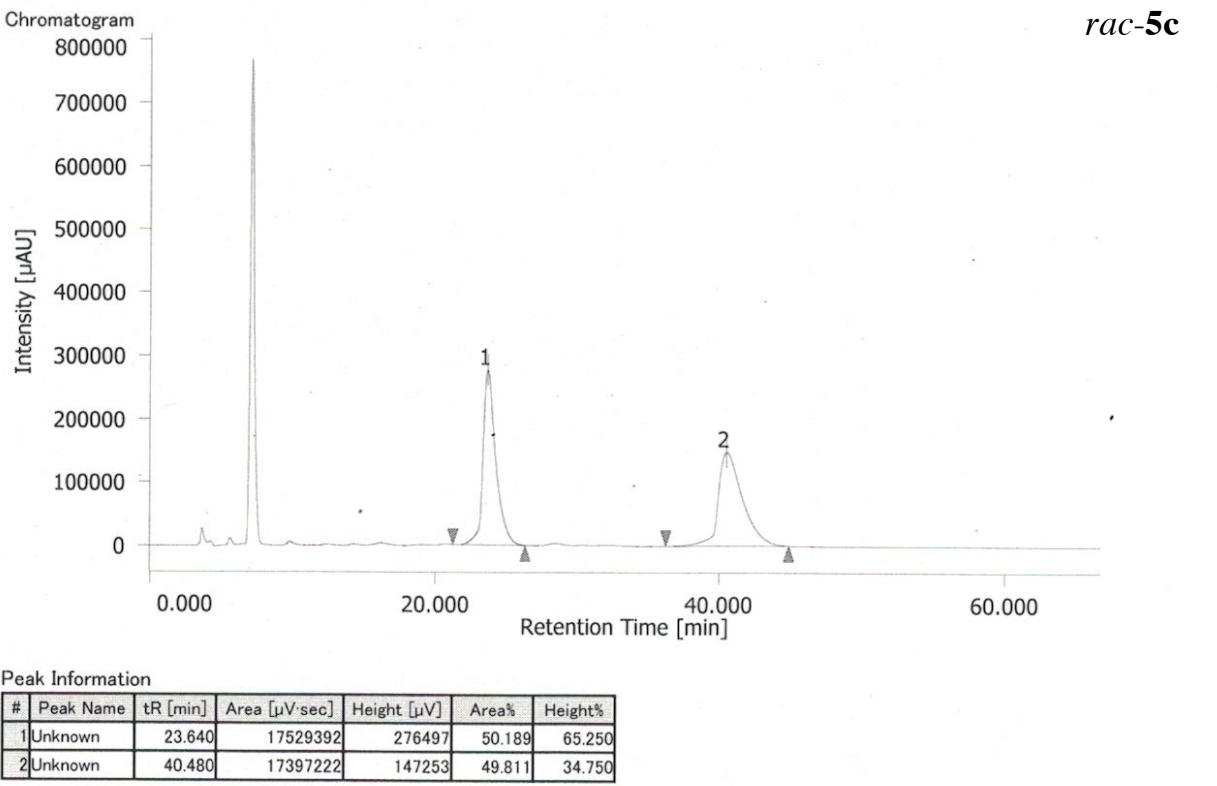


HPLC spectra of 5d and rac-5d

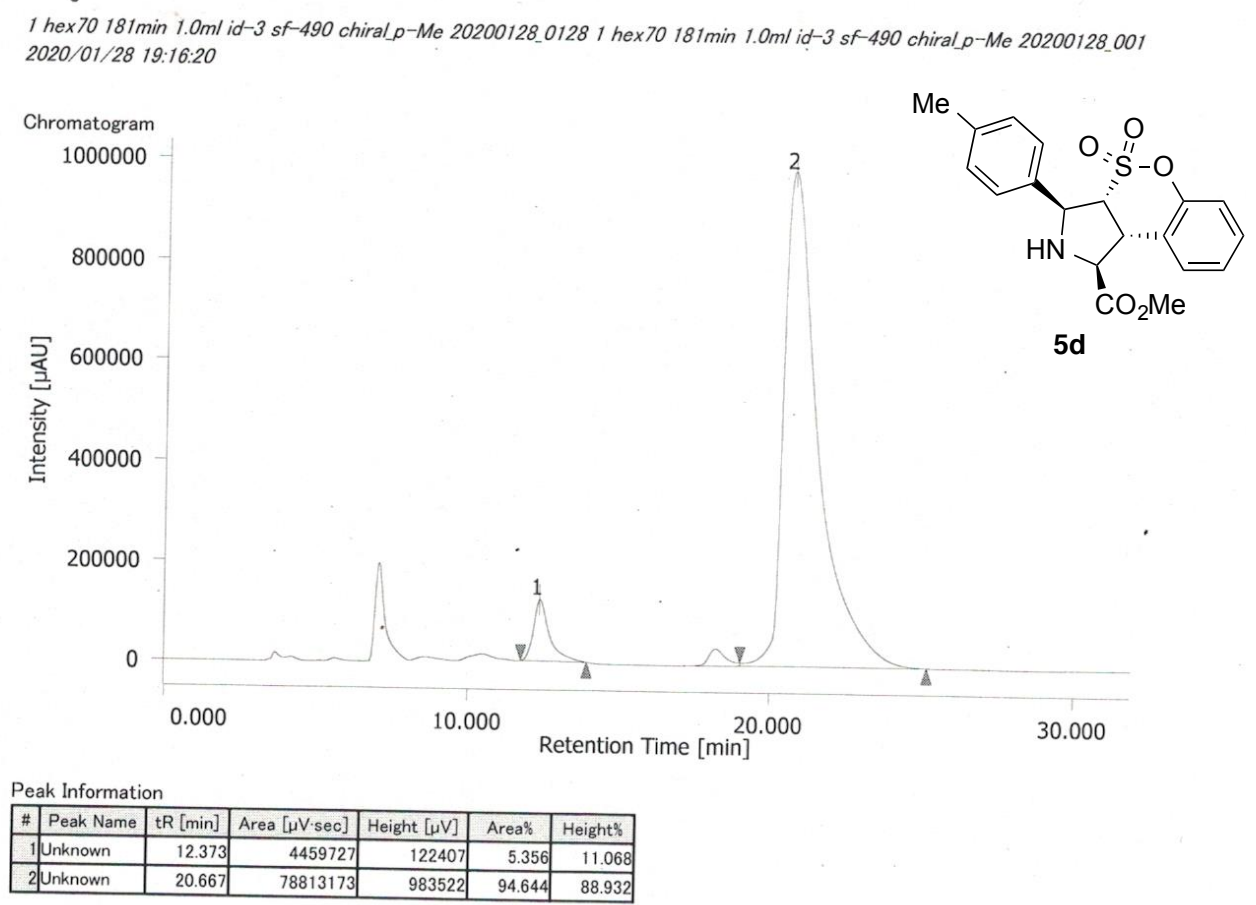

1 hex70 $181 \mathrm{~min} 1.0 \mathrm{ml}$ id-3 sf-444 rac_p-Me_1219 1 hex70 181 min 1.0m/ id-3 sf-444 rac_p-Me_001 2019/12/21 14:38:18

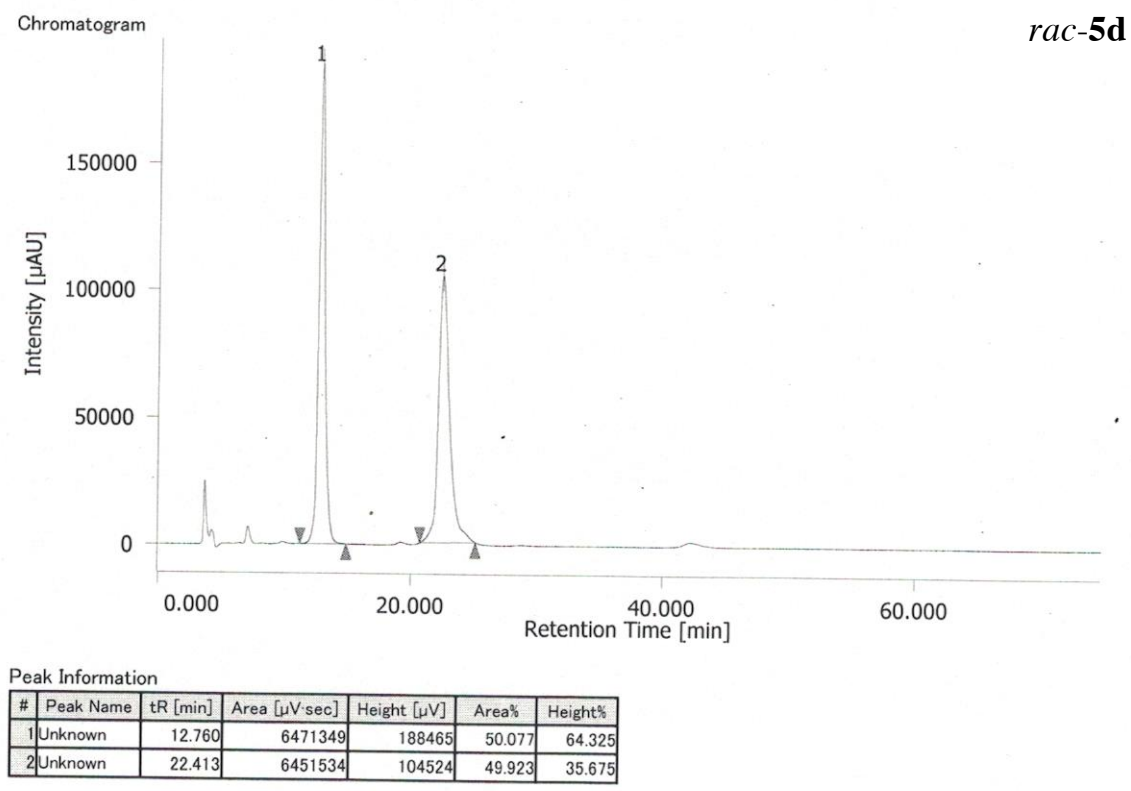


HPLC spectra of $5 \mathbf{e}$ and $r a c-5 e$
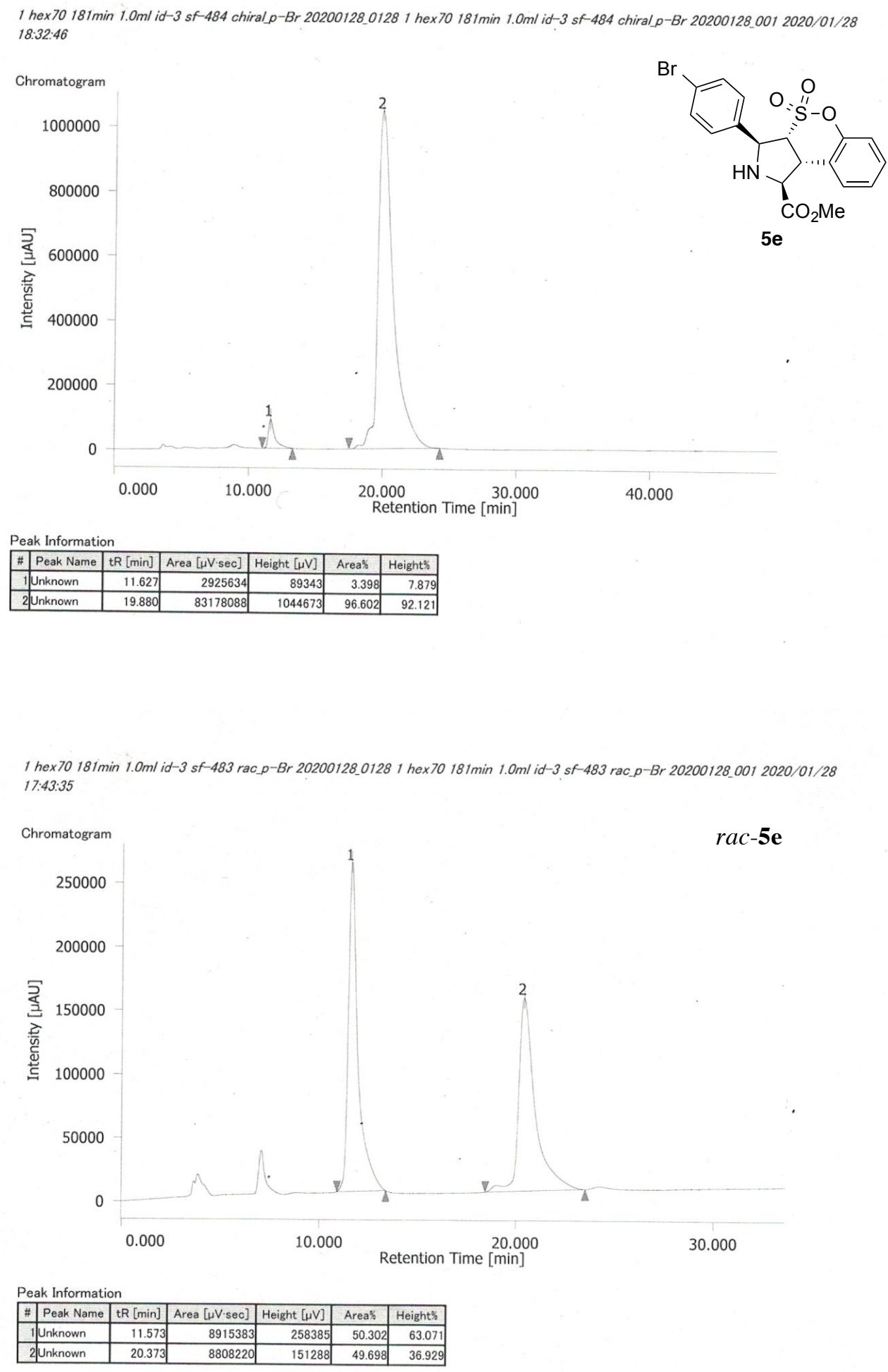
HPLC spectra of $\mathbf{5 f}$ and $r a c-5 f$

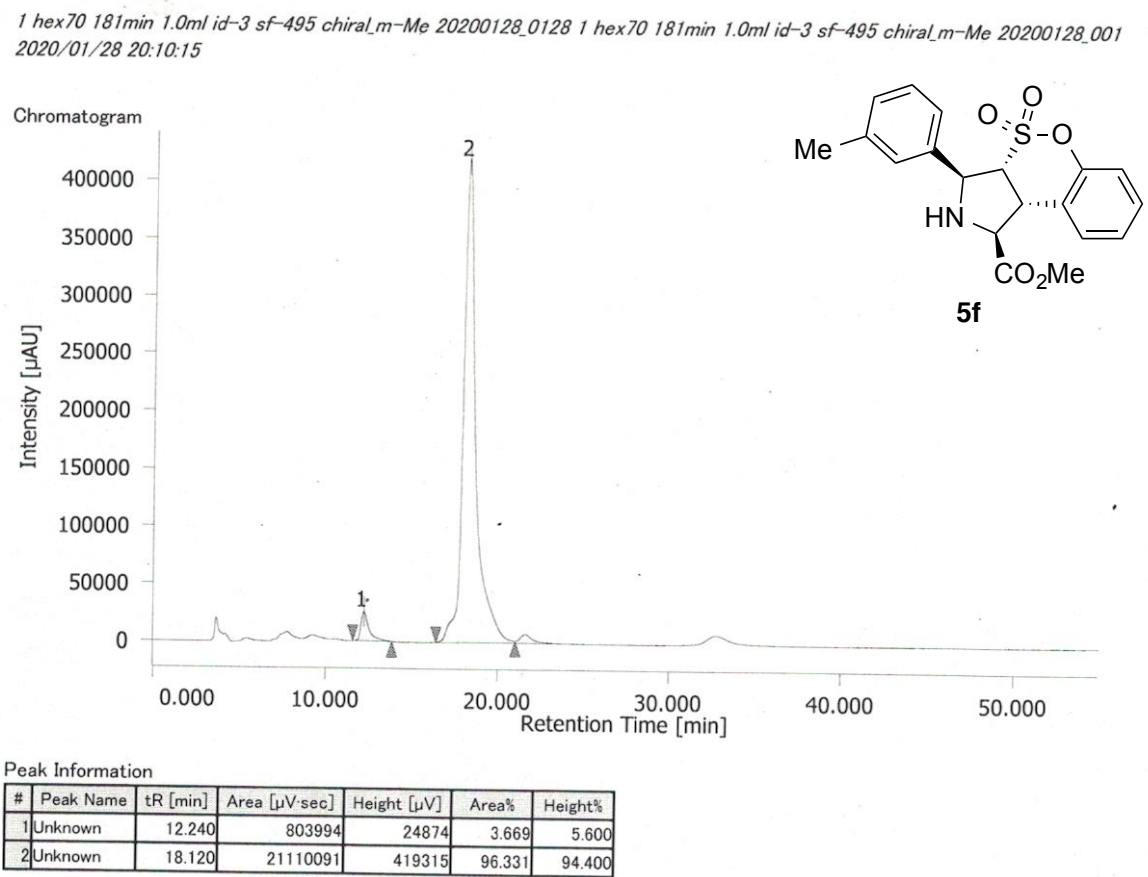

1 hex70 18tmin 1.0m/ id-3 sf-445 rac_m-Me 20191221_1221 1 hex70 181min 1.0ml id-3 sf-445 rac_m-Me 20191221 001 2019/12/21 15:09:11

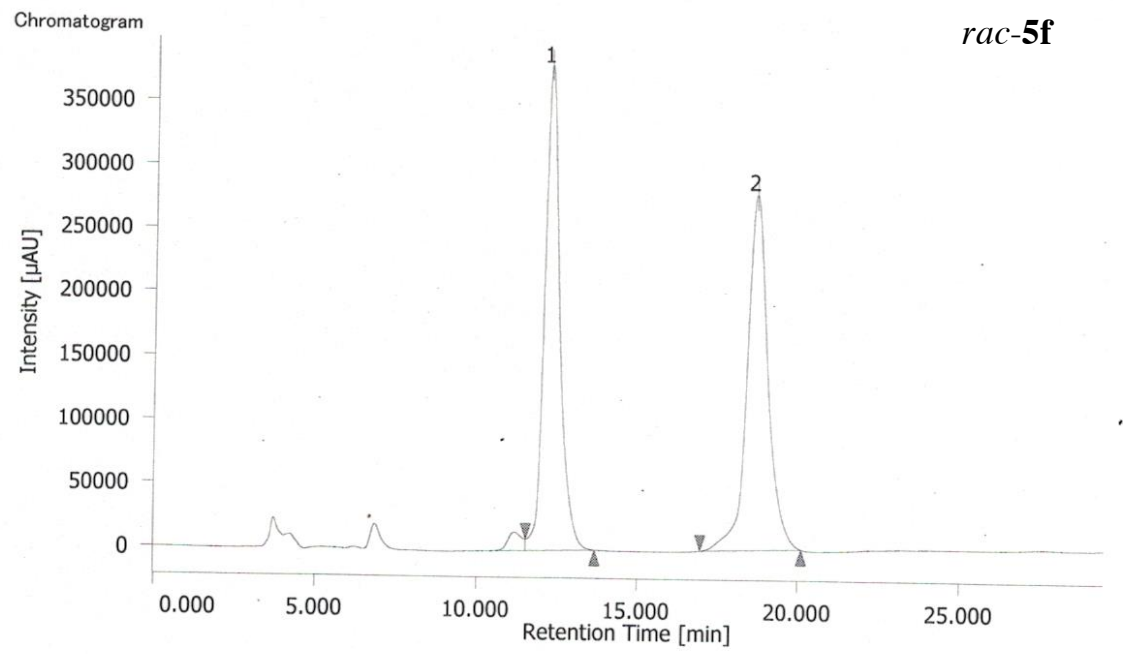

Peak Information

\# \begin{tabular}{l|r|r|r|r|r|} 
Peak Name & tR $[\mathrm{min}]$ & Area $[\mu \mathrm{V} \cdot \mathrm{sec}]$ & Height $[\mu \mathrm{V}]$ & Area\% & Height\% \\
\hline
\end{tabular}

\begin{tabular}{|l|r|r|r|r|r|}
\hline 1 Unknown & 12.187 & 12477325 & 379768 & 49.959 & 57.75 \\
\hline
\end{tabular}

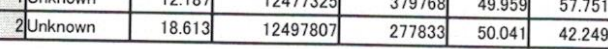


HPLC spectra of $\mathbf{5 g}$ and $r a c-5 \mathbf{g}$

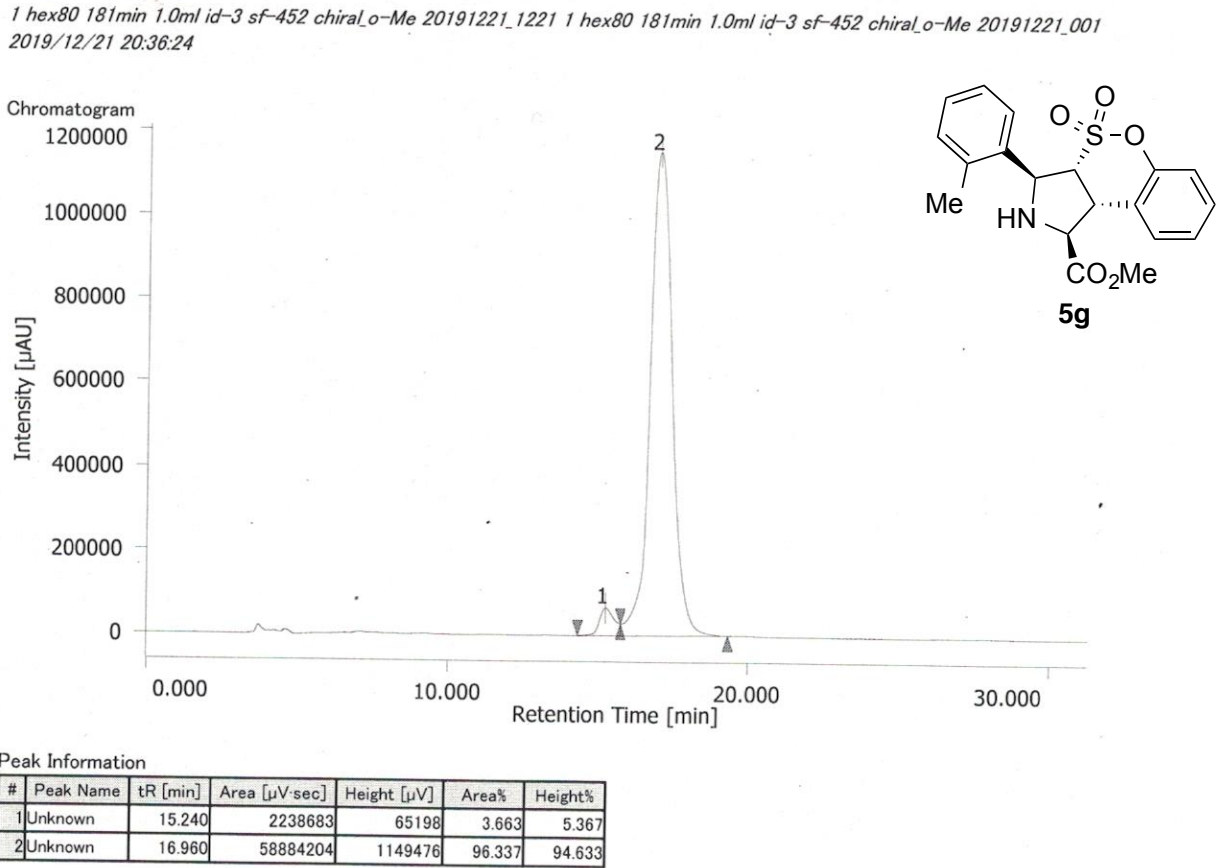

1 hex80 181min 1.0m/ id-3 sf-446 rac_o-Me 20191221_1221 1 hex80 $181 \mathrm{~min}$ 1.0ml id-3 sf-446 o-Me 20191221_001 2019/12/21 20:04:46

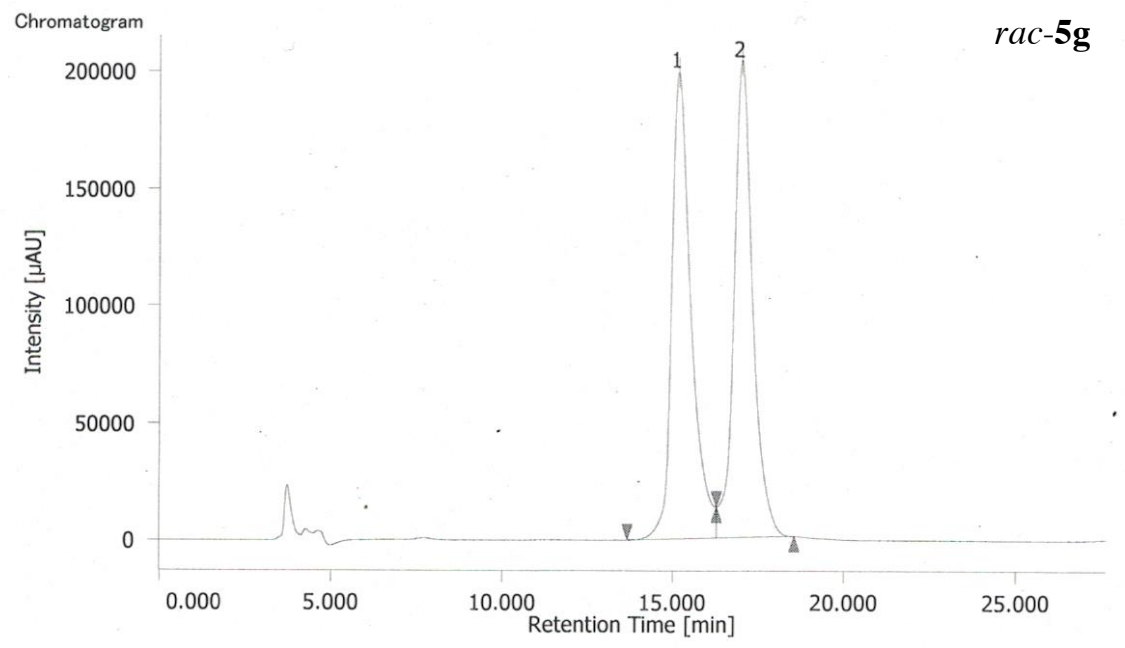

Peak Information

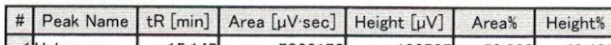

\begin{tabular}{|l|r|r|r|r|r|}
\hline U Unknown & 15.147 & 7866172 & 198737 & 50.363 & 49.405 \\
\hline
\end{tabular}

\begin{tabular}{|l|r|r|r|r|r|}
\hline 2 Unknown & 16.987 & 7752688 & 203527 & 49.637 & 50.595 \\
\hline
\end{tabular} 
HPLC spectra of $\mathbf{5 h}$ and rac-5h
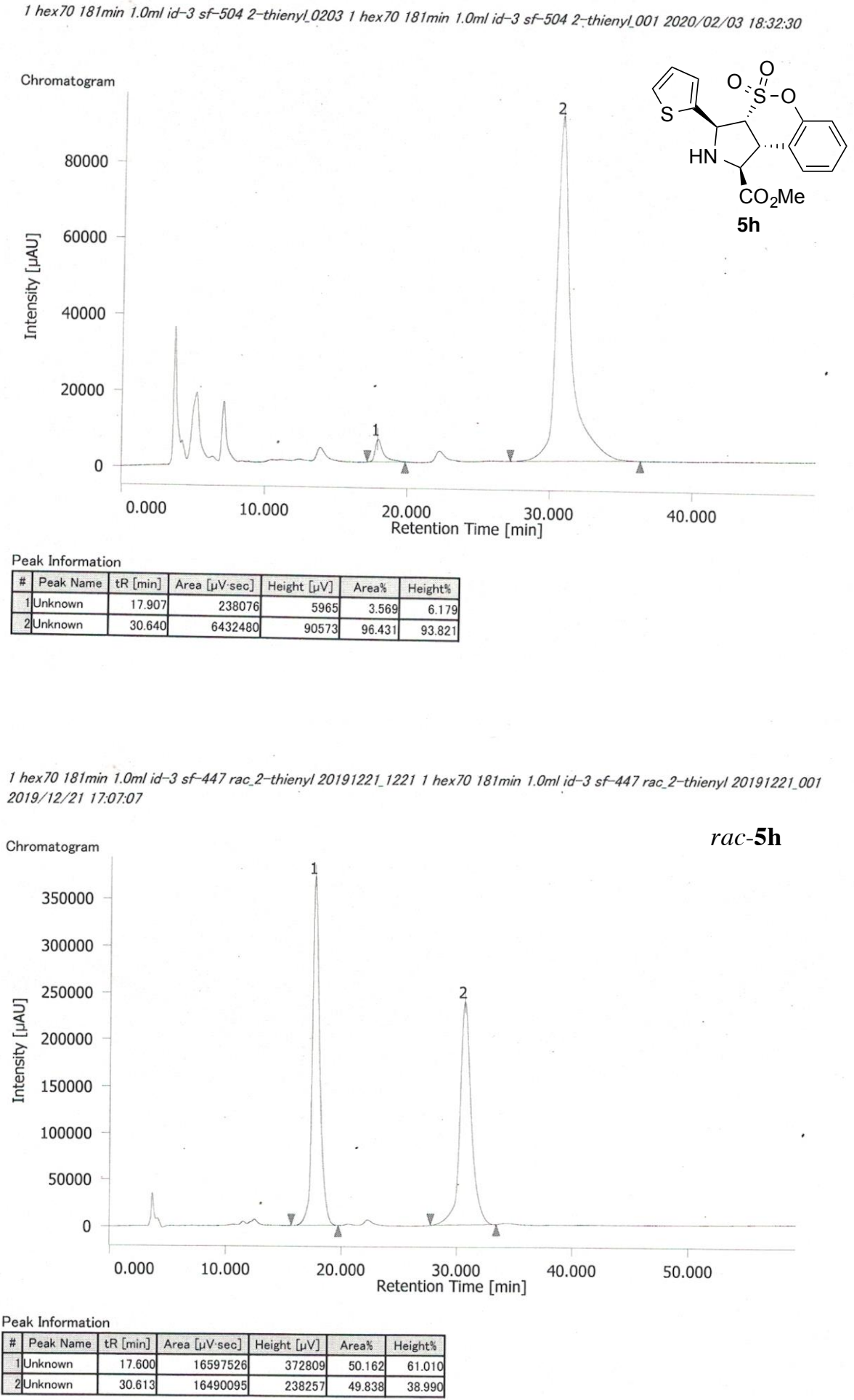
HPLC spectra of $\mathbf{5 i}$ and $r a c-5 i$

1 hex70 181min 1.0m/ id-3 sf-505 6-OMe_0203 1 hex70 $181 \mathrm{~min} 1.0 \mathrm{ml}$ id-3 sf-505 6-OMe_001 2020/02/08 17:21:48

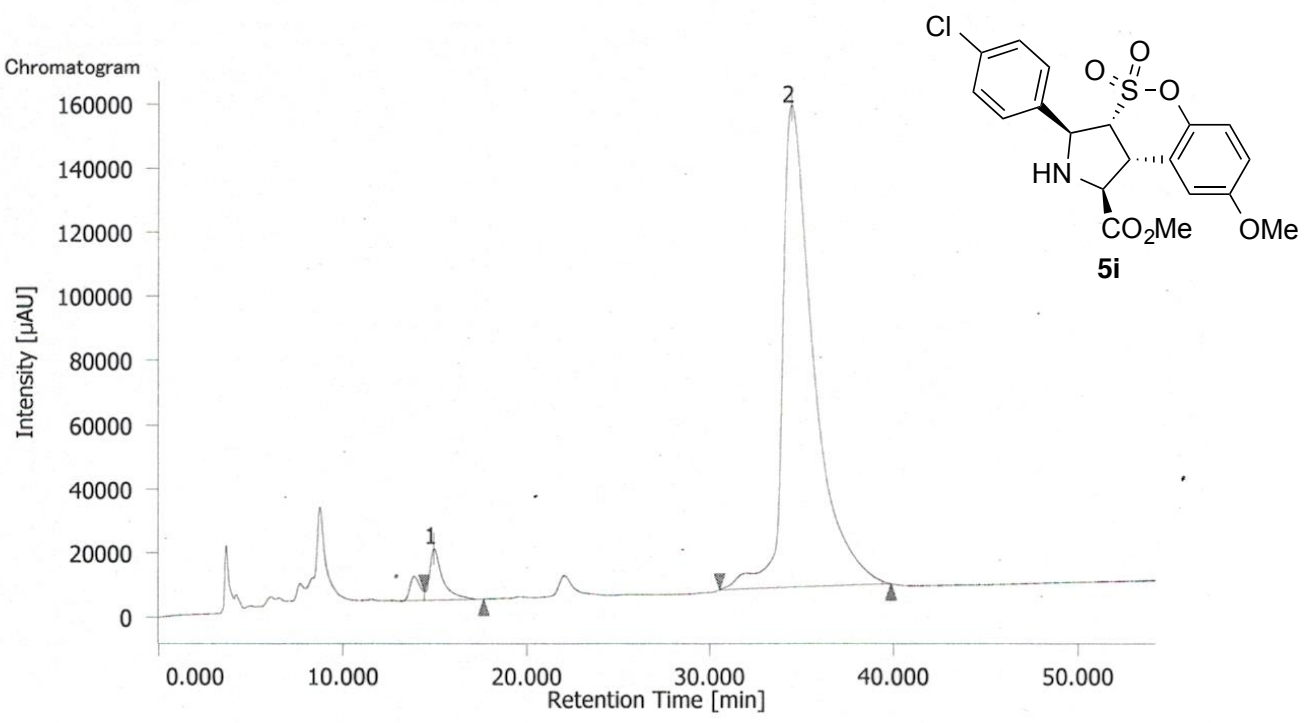

Peak Information

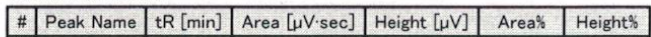

\begin{tabular}{|l|r|r|r|r|r|}
\hline 1 Unknown & 14.947 & 778955 & 16025 & 4.206 & 9.647 \\
\hline 2 Unknown & 34.400 & 17740777 & 150097 & 95.794 & 90.353 \\
\hline
\end{tabular}

1 hex70 $181 \mathrm{~min} 1.0 \mathrm{ml}$ id-3 sf-418_rac-p-Cl_6-OMe_1125 1 hex70 $181 \mathrm{~min} 1.0 \mathrm{ml}$ id-3 sf-418_rac-p-Cl_6-OMe_001 2019/12/21 14:46:07

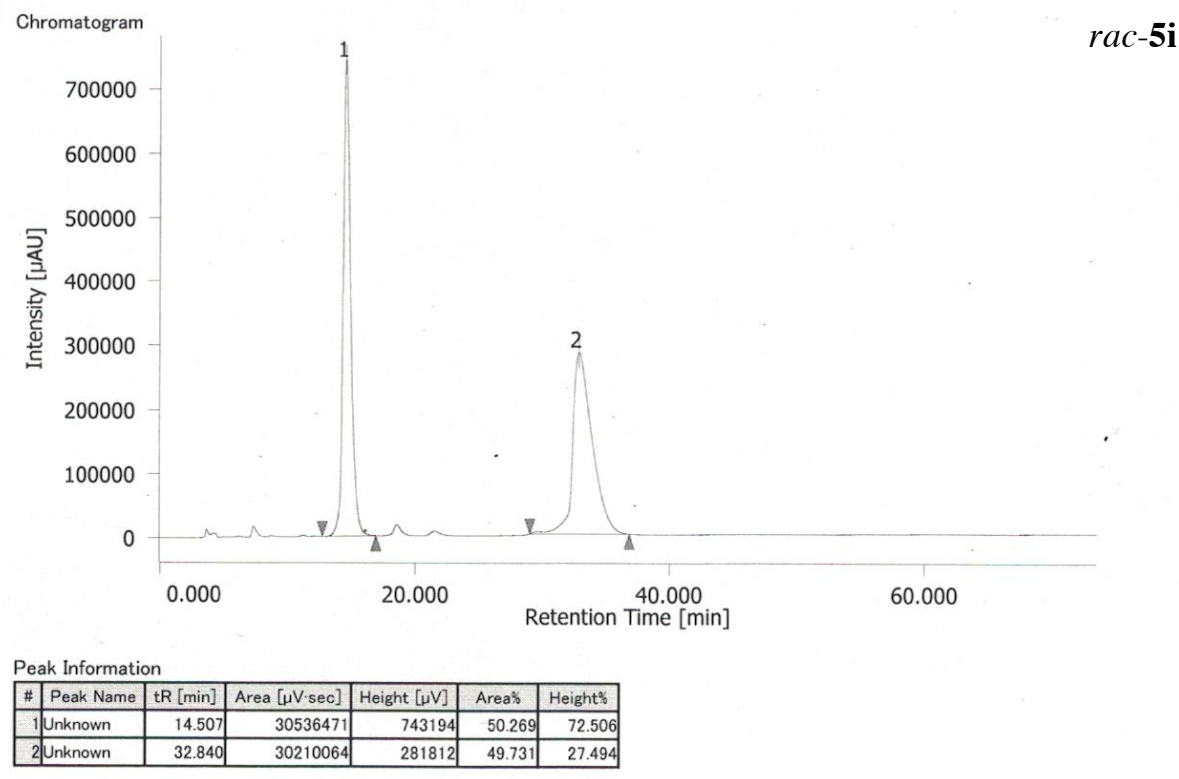


HPLC spectra of $\mathbf{5 j}$ and $r a c-\mathbf{5} \mathbf{j}$
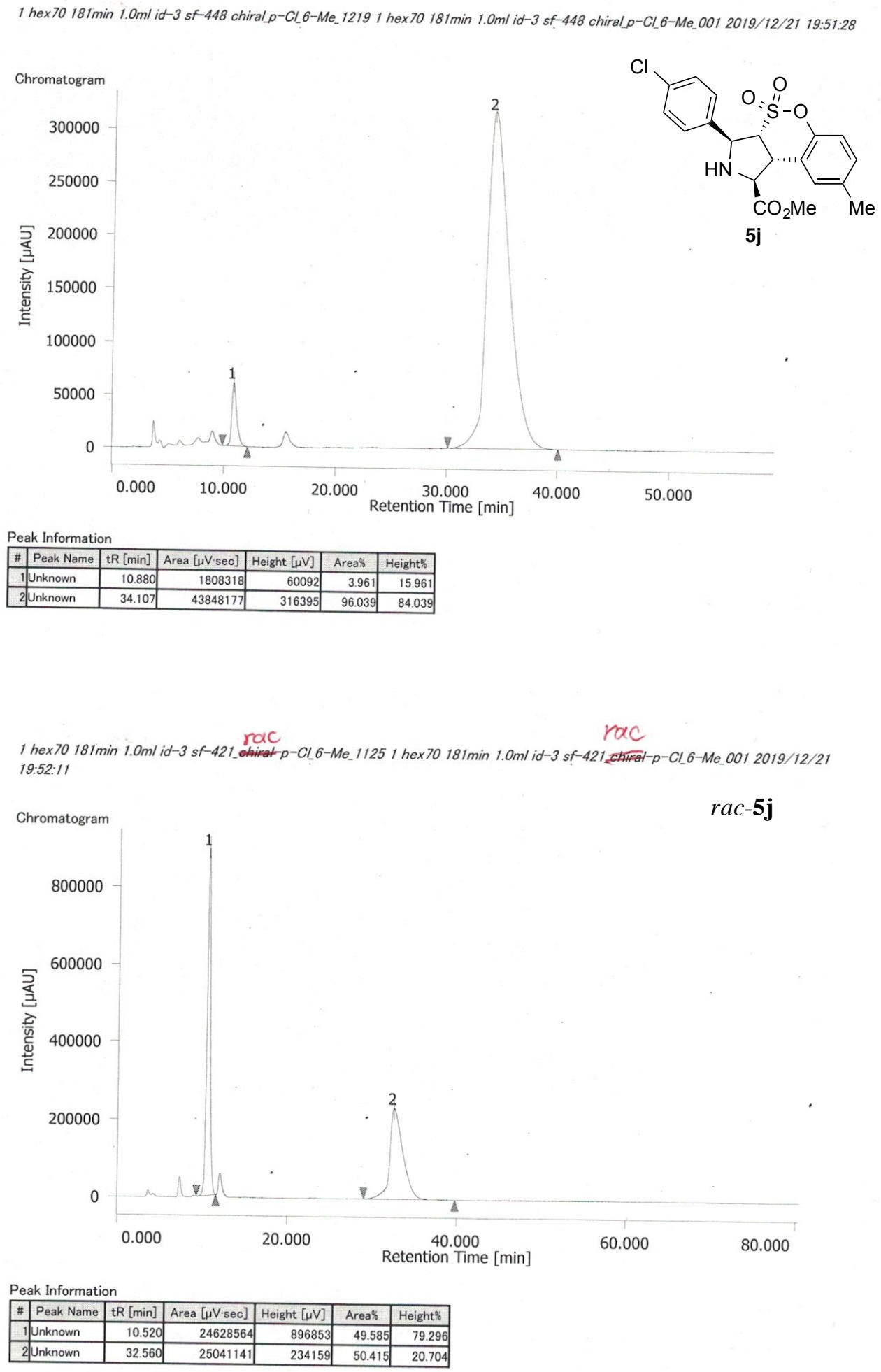
HPLC spectra of $\mathbf{5 k}$ and rac-5k

1 hex70 $181 \mathrm{~min} 1.0 \mathrm{ml}$ id-3 sf-419_chiral-p-Cl_6-Cl_1125 1 hex70 $181 \mathrm{~min} 1.0 \mathrm{ml}$ id-3 sf-419_chiral-p-Cl_6-Cl_001 2019/12/21 14:44:42

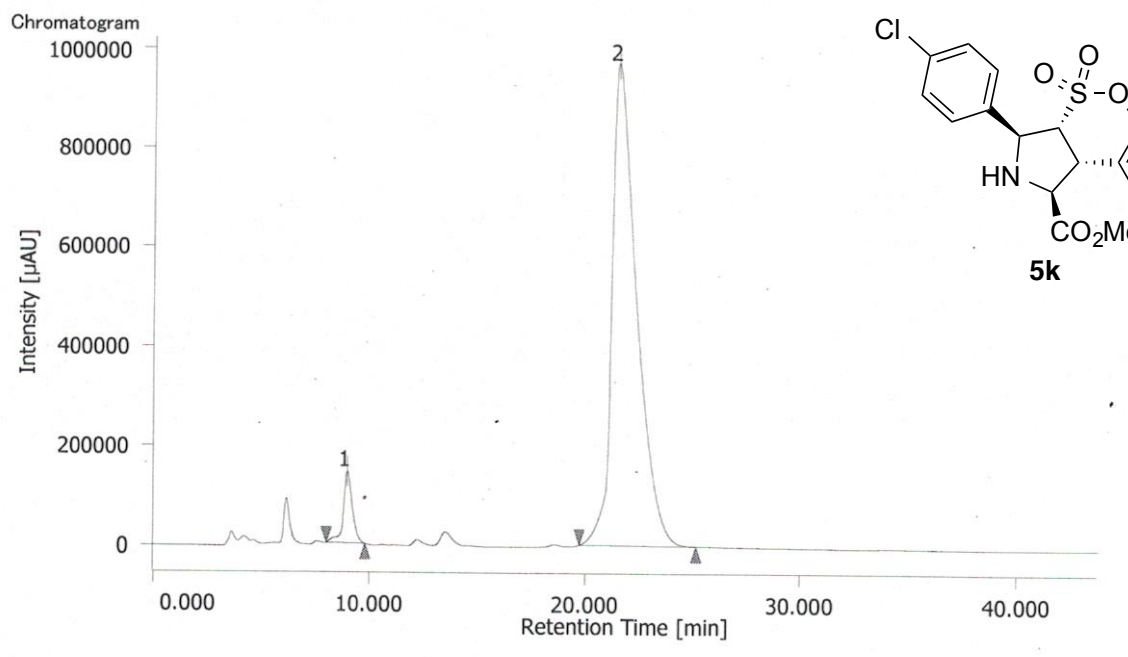

Peak Information

\begin{tabular}{|l|l|r|r|r|r|r|}
\hline$\#$ & Peak Name & tR $[\mathrm{min}]$ & Area $[\mu \mathrm{V} \cdot \mathrm{sec}]$ & Height $[\mu \mathrm{V}]$ & Area\% & Height/\% \\
\hline 1 & Unknown & 8.960 & 4086743 & 142868 & 5.008 & 1286 \\
\hline
\end{tabular}

\begin{tabular}{|l|r|r|r|r|r|}
\hline 1Unknown & 8.960 & 4086743 & 142868 & 5.008 & 12.866 \\
\hline 2Unknown & 21.467 & 77510661 & 967591 & 94.992 & 87.134 \\
\hline
\end{tabular}

$1 \mathrm{hex} 70181 \mathrm{~min} 1.0 \mathrm{ml}$ id-3 sf-413_rac-p-Cl_6-Cl $11251 \mathrm{hex} 70181 \mathrm{~min} 1.0 \mathrm{ml}$ id-3 sf-413_rac-p-Cl_6-Cl_oo1 2019/12/21 14:45:29

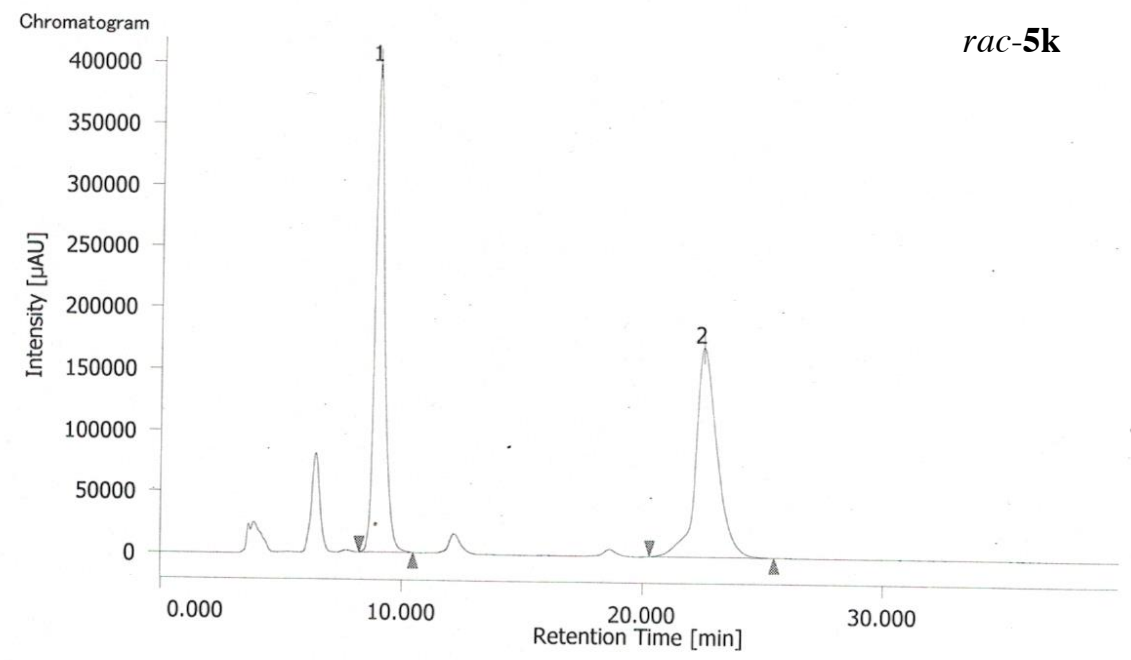

Peak Information

\begin{tabular}{|l|l|r|r|r|r|r|}
\hline$\#$ & Peak Name & tR [min] & Area $[\mu \mathrm{V} \cdot \mathrm{sec}]$ & Height $[\mu \mathrm{V}]$ & Area\% & Height\% \\
\hline
\end{tabular}

\begin{tabular}{|l|r|r|r|r|r|}
\hline 1 Unknown & 8.933 & 10718726 & 397862 & 50.428 & 70.045 \\
\hline
\end{tabular}

\begin{tabular}{|l|r|r|r|r|r|}
\hline 2 Unknown & 22.507 & 10536842 & 170150 & 49.572 & 29.955 \\
\hline
\end{tabular}


HPLC spectra of $\mathbf{5 l}$ and $r a c-5 \mathbf{l}$

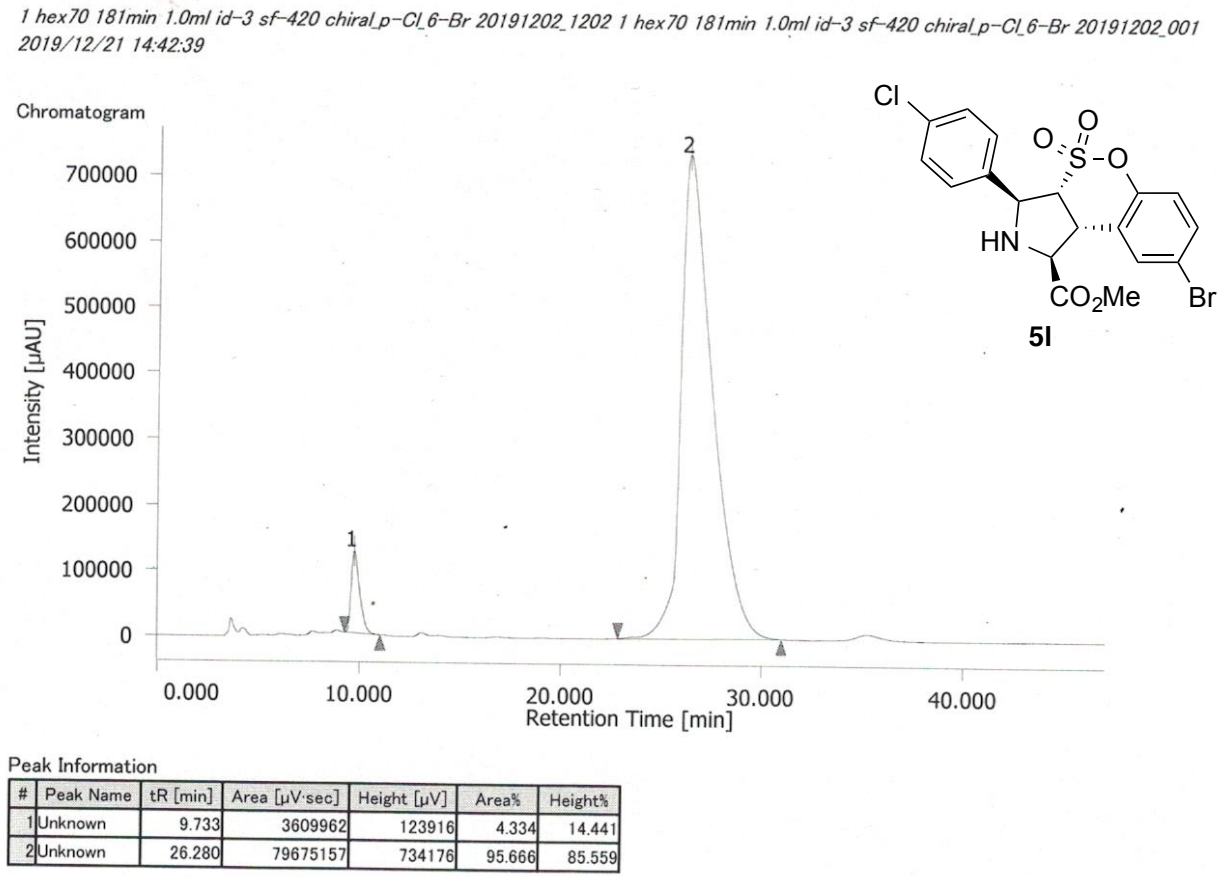
1 hex70 $181 \mathrm{~min} 1.0 \mathrm{ml}$ id-3 sf-414 rac_p-Cl_6-Br 20191202.12021 hex70 $181 \mathrm{~min} 1.0 \mathrm{ml}$ id-3 sf-414 rac_p-Cl_6-Br 20191202_001
$2019 / 12 / 21$ 14:43:20

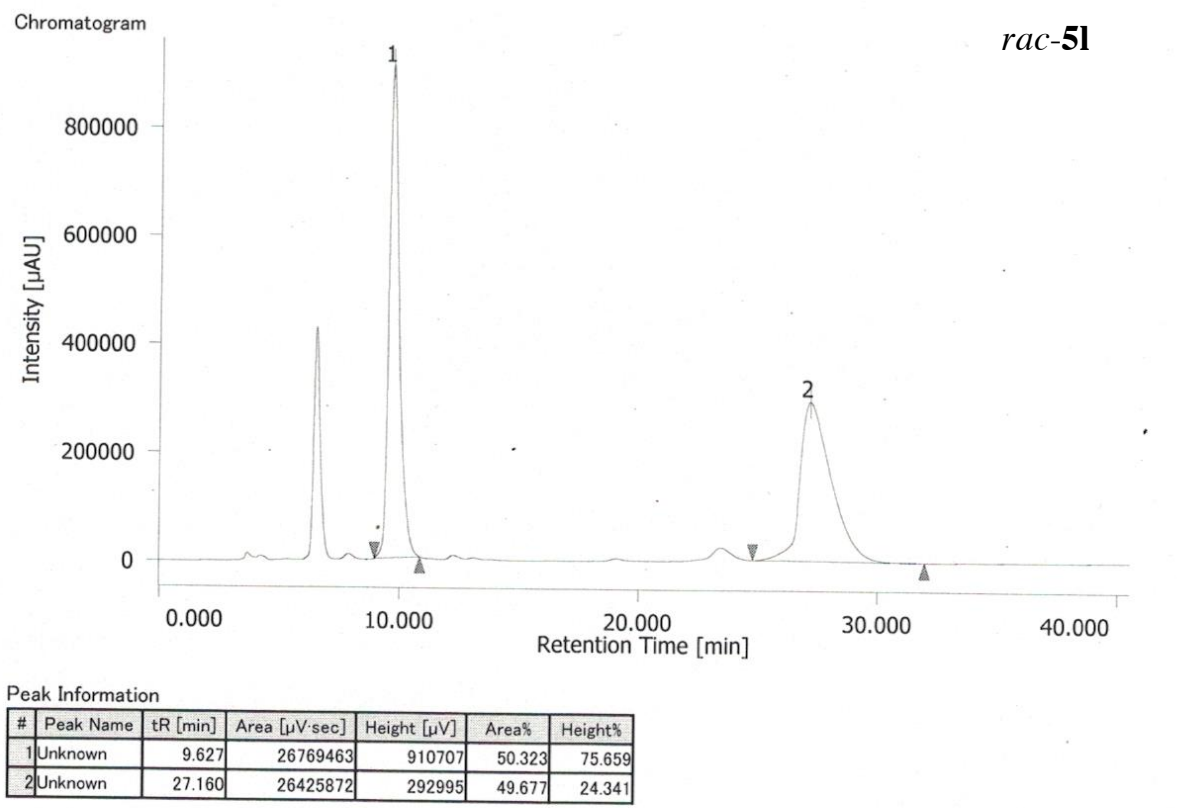




\section{X-Ray Crystal Structure Analysis}

Single crystals of $\mathbf{4 l}$ and $\mathbf{5 h}$ were crystallized from dichloromethane/acetonitrile by vapor diffusion technique using hexane as an antisolvent. A suitable single crystal was selected and mounted on the glass fiber and transferred to the goniometer of a Rigaku VariMax Saturn CCD diffractometer with graphite-monochromated Mo K $\alpha$ radiation $(\lambda=71.073 \mathrm{pm})$. Yadokari-XG 2009 program $^{1}$ was used as a graphical interface. The structure was solved and refined by SIR-2004 ${ }^{2}$ by SHELX-97 programs. $^{3}$

The refinement was performed on anisotropically for all non-hydrogen atoms. Hydrogen atoms were placed using AFIX instructions.

Figure S1. Thermal ellipsoid (50 \% probability) plot of 41, CCDC No. 1993601

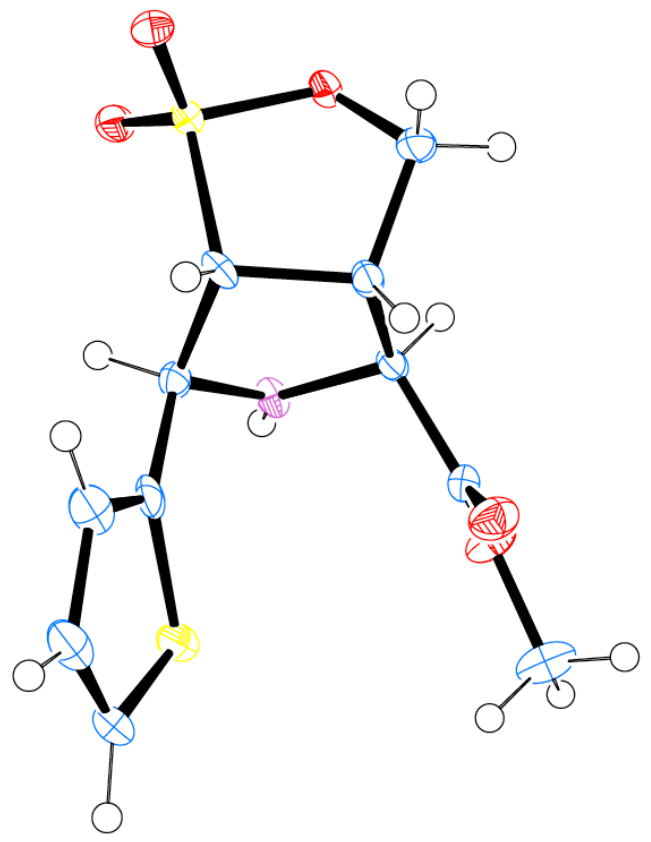


Table S1. Crystal data and structure refinements for $\mathbf{4 l}$

\begin{tabular}{|c|c|}
\hline Empirical formula & $\mathrm{C}_{11} \mathrm{H}_{13} \mathrm{NO}_{5} \mathrm{~S}_{2}$ \\
\hline Formula weight & 303.35 \\
\hline Space system & orthorhombic \\
\hline Space group & P $2 a c 2 a b$ \\
\hline $\mathrm{a} / \AA ̊$ & $6.043(2)$ \\
\hline $\mathrm{b} / \AA$ & $7.662(2)$ \\
\hline $\mathrm{c} / \AA ̊$ & $27.127(8)$ \\
\hline$\alpha /^{\circ}$ & 90 \\
\hline$\beta /^{\circ}$ & 90 \\
\hline$\gamma /{ }^{\circ}$ & 90 \\
\hline Volume $/ \AA^{3}$ & $1256.0(7)$ \\
\hline $\mathrm{Z}$ & 4 \\
\hline Temperature/K & 93 \\
\hline $2 \Theta$ range for data collection ${ }^{\circ}$ & 3.4542 to 31.4970 \\
\hline$\rho_{\text {calcd }} \mathrm{g} / \mathrm{m}^{3}$ & 1.604 \\
\hline$\mu / \mathrm{mm}^{-1}$ & 0.440 \\
\hline F_000 & 632 \\
\hline Crystal_size $/ \mathrm{mm}^{3}$ & $0.240 \times 0.200 \times 0.050$ \\
\hline Radiation & $\operatorname{MoK} \alpha$ \\
\hline Reflections collected & 2619 \\
\hline Independent & 8075 \\
\hline Index ranges & $-7 \leqq \mathrm{~h} \leqq 7,-9 \leqq \mathrm{k} \leqq 9,-31 \leqq 1 \leqq 35$ \\
\hline Flack & $-0.06(6)$ \\
\hline Absolute configuration & $\mathrm{ad}$ \\
\hline Data/restraints/parameters & $2619 / 0 / 172$ \\
\hline Final R indexes R $[\mathrm{I}>2 \sigma(\mathrm{I})] \mathrm{gt}$ & $\mathrm{R}_{1}=0.0574, \mathrm{wR}_{2}=0.1470$ \\
\hline Final $\mathrm{R}$ indexes $\mathrm{R}$ [all data] & $\mathrm{R}_{1}=0.0675, \mathrm{wR}_{2}=0.1577$ \\
\hline Goodness-of-fit on $\mathrm{F}^{2}$ & 1.050 \\
\hline Largest peak/deepest hole e $\AA^{-3}$ & $0.479 /-0.692$ \\
\hline
\end{tabular}


Figure S2. Thermal ellipsoid (50 \% probability) plot of $\mathbf{5 h}$, CCDC No. 199360

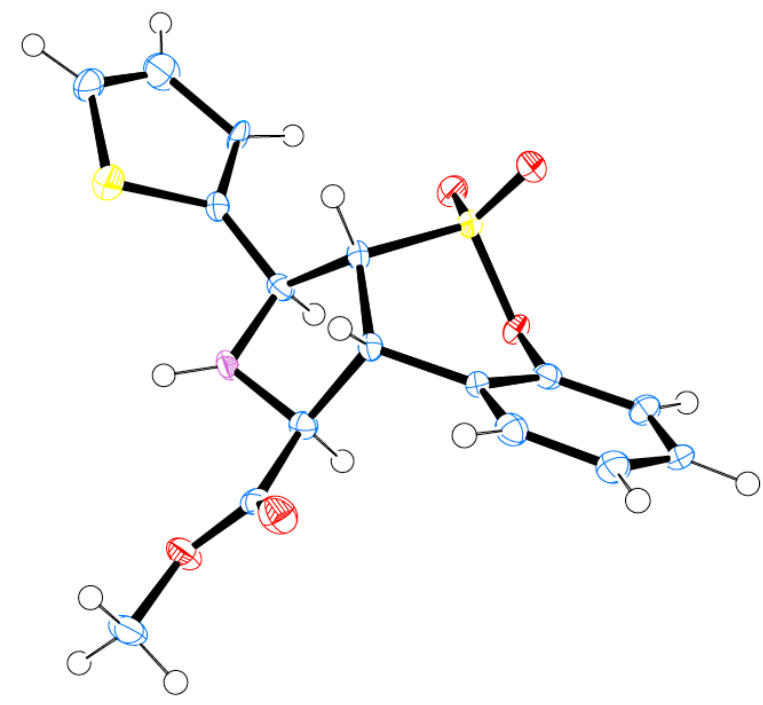


Table S2. Crystal data and structure refinements for $\mathbf{5 h}$

\begin{tabular}{|c|c|}
\hline Empirical formula & $\mathrm{C}_{16} \mathrm{H}_{15} \mathrm{NO}_{5} \mathrm{~S}_{2}$ \\
\hline Formula weight & 365.42 \\
\hline Space system & orthorhombic \\
\hline Space group & P $2 a c 2 a b$ \\
\hline $\mathrm{a} / \AA$ & $8.5122(15)$ \\
\hline $\mathrm{b} / \AA$ & $9.4305(15)$ \\
\hline $\mathrm{c} / \AA$ & $20.019(4)$ \\
\hline$\alpha /{ }^{\circ}$ & 90 \\
\hline$\beta /{ }^{\circ}$ & 90 \\
\hline$\gamma /{ }^{\circ}$ & 90 \\
\hline Volume $/ \AA^{3}$ & $1607.0(5)$ \\
\hline Z & 4 \\
\hline Temperature/K & 93 \\
\hline $2 \Theta$ range for data collection $/{ }^{\circ}$ & 2.9678 to 31.0999 \\
\hline$\rho_{\text {calcd }} g / \mathrm{m}^{3}$ & 1.510 \\
\hline$\mu / \mathrm{mm}^{-1}$ & 0.358 \\
\hline F_000 & 760 \\
\hline Crystal_size $/ \mathrm{mm}^{3}$ & $0.250 \times 0.160 \times 0.070$ \\
\hline Radiation & $\operatorname{MoK} \alpha$ \\
\hline Reflections collected & 3665 \\
\hline Independent & 13161 \\
\hline Index ranges & $-11 \leqq \mathrm{~h} \leqq 11,-12 \leqq \mathrm{k} \leqq 10,-24 \leqq 1 \leqq 25$ \\
\hline Flack & $-0.01(4)$ \\
\hline Absolute configuration & $\mathrm{ad}$ \\
\hline Data/restraints/parameters & $3665 / 0 / 217$ \\
\hline Final $\mathrm{R}$ indexes $\mathrm{R}[\mathrm{I}>2 \sigma(\mathrm{I})] \mathrm{gt}$ & $\mathrm{R}_{1}=0.0461, \mathrm{wR}_{2}=0.1136$ \\
\hline Final $\mathrm{R}$ indexes $\mathrm{R}$ [all data] & $\mathrm{R}_{1}=0.0535, \mathrm{wR}_{2}=0.1241$ \\
\hline Goodness-of-fit on $\mathrm{F}^{2}$ & 1.047 \\
\hline Largest peak/deepest hole e $\AA^{-3}$ & $0.464 /-0.611$ \\
\hline
\end{tabular}




\section{References for supporting information}

1. C. Kabuto, S. Akine, T. Nemoto, E. Kwon, J. Cryst. Soc. Jpn. 2009, 51, 218-224.

2. M. C. Burla, R. Caliandro, M. Camalli, B. Carrozzini, G. L. Cascarano, L. D. Caro, C. Giacovazzo, G. Polidori, R. Spagna, J. Appl. Cryst. 2005, 38, 381-388.

3. G. M. Sheldrick, Acta Crystallogr. Sect. A, 2008, 64, 112-122. 